\title{
Development of Radar Navigation and Radio Data Transmission for Microhole Coiled Tubing Bottom Hole Assemblies
}

\section{Final Technical Report}

Reporting Period Start Date:

Reporting Period End Date:

Principal Authors:

Date Report Issued:

DOE Award Number:

Name and Address of Submitting Organization:
July 2004

March 2007

Larry G. Stolarczyk

Gerald L. Stolarczyk

Larry Icerman

John Howard

Hooman Tehrani

December 2007

DE-FC26-04NT15477

Stolar Research Corporation 848 Clayton Highway

Raton, New Mexico 87740 


\section{$\underline{\text { Disclaimer }}$}

This report was prepared as an account of work sponsored by an agency of the United States Government. Neither the United States Government nor any agency thereof, nor any of their employees, makes any warranty, express or implied, or assumes any legal liability or responsibility for the accuracy, completeness, or usefulness of any information, apparatus, product, or process disclosed, or represents that its use would not infringe privately owned rights. Reference herein to any specific commercial product, process, or service by trade name, trademark, manufacturer, or otherwise does not necessarily constitute or imply its endorsement, recommendation, or favoring by the United States Government or any agency thereof. The views and opinions of authors expressed herein do not necessarily state or reflect those of the United States Government or any agency thereof. 


\begin{abstract}
$\underline{\text { Abstract }}$
This Final Technical Report summarizes the research and development (R\&D) work performed by Stolar Research Corporation (Stolar) under U.S. Department of Energy (DOE) Contract Number DE-FC26-04NT15477. This work involved the development of radar navigation and radio data transmission systems for integration with microhole coiled tubing bottom hole assemblies. Under this contract, Stolar designed, fabricated, and laboratory and field tested two advanced technologies of importance to the future growth of the U.S. oil and gas industry: (i) real-time measurement-while-drilling (MWD) for guidance and navigation of coiled tubing drilling in hydrocarbon reservoirs and (ii) two-way inductive radio data transmission on coiled tubing for real-time, subsurface-to-surface data transmission. The operating specifications for these technologies are compatible with 3.5-inch boreholes drilled to a true vertical depth (TVD) of 5,000 feet, which is typical of coiled tubing drilling applications.

These two technologies (i.e., the Stolar Data Transmission System and Drill String Radar) were developed into pre-commercial prototypes and tested successfully in simulated coiled tubing drilling conditions. Integration of these two technologies provides a real-time geosteering capability with extremely quick response times. Stolar is conducting additional work required to transition the Drill String Radar into a true commercial product. The results of this advanced development work should be an important step in the expanded commercialization of advanced coiled tubing microhole drilling equipment for use in U.S. hydrocarbon reservoirs.
\end{abstract}




\section{Table of Contents}

List of Graphical Materials $\ldots \ldots \ldots \ldots \ldots \ldots$

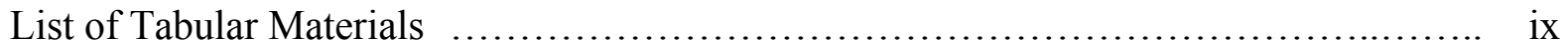

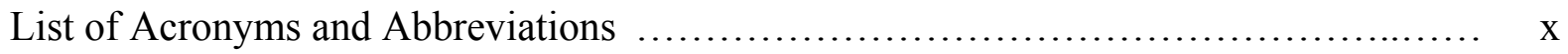

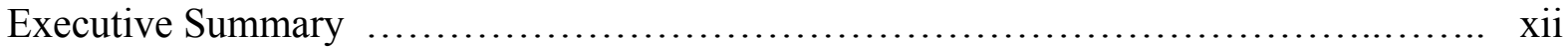

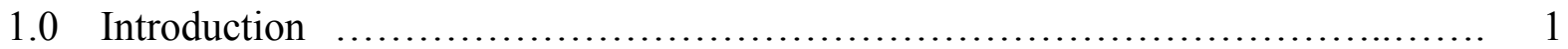

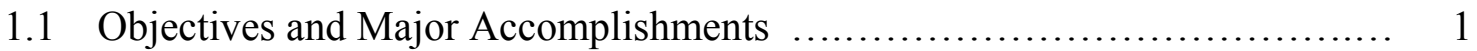

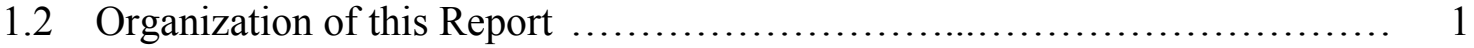

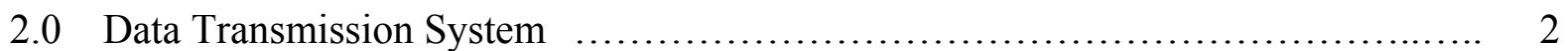

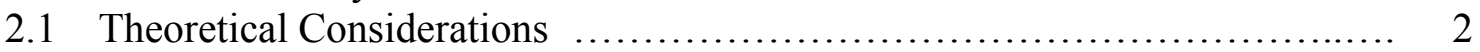

2.2 First-Generation Prototype Data Transmission System f..................... 4

2.2.1 Experimental Design of the First-Generation Prototype .............. 4

2.2.2 Experimental Results for the First-Generation Prototype .............. 6

2.3 Experimental Design of the Second-Generation Prototype Data

Transmission System ................................................... 8

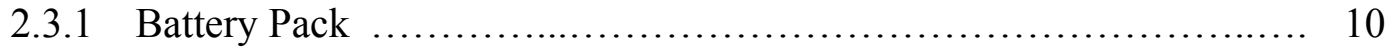

2.3.2 Electronics ..................................................... 11

2.3.3 Antennas ......................................................... 11

2.3.4 Antenna Performance Modeling ................................. 13

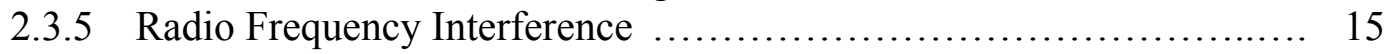

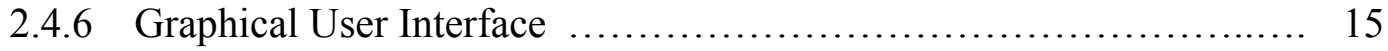

2.4 Coal-Based Applications of the Prototype Data Transmission System ....... 15

2.5 Conclusions ............................................................. 16

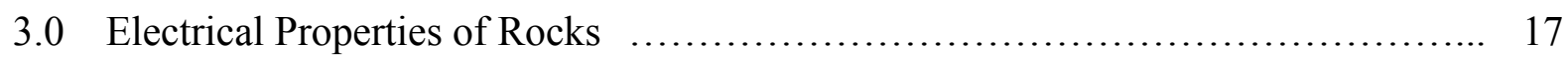

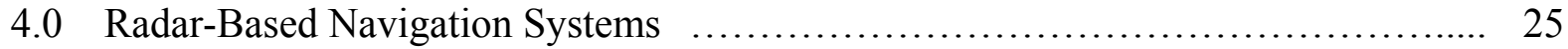

4.1 Theoretical Considerations .............................................. 25

4.1.1 Basic Radar Background ....................................... 25

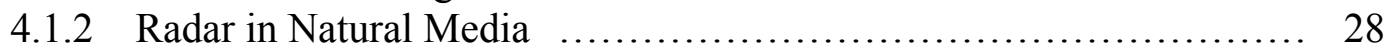

4.1.3 Stepped-Frequency, Continuous-Wave Radar ...................... 32

4.1.4 Frequency-Modulated, Continuous-Wave Radar .................... 33

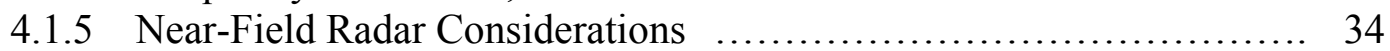

4.1.6 Advanced Radar Design ........................................... 36

4.2 Prototype Mid-Seam Guidance Tool …................................ 41

4.2.1 Electromagnetic Gradiometer Background ........................ 41

4.2.2 Experimental Design of the Prototype Mid-Seam Guidance Tool … 42

4.2.3 Experimental Results for the Prototype Mid-Seam Guidance Tool ... 44

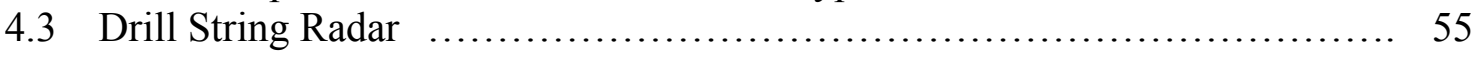


4.3.1 Background .............................................. 55

4.3.2 Theoretical Description of the Prototype Drill String Radar Tool ..... 60

4.3.3 Experimental Design of the Prototype Drill String Radar Tool ........ 62

4.3.4 Experimental Results for the Prototype Drill String Radar Tool ....... 67

4.3.4.1 Field Test Description ................................. 67

4.3.4.2 Field Testing Procedures ............................. 72

4.3.4.3 Analysis of the Collected Data .......................... 73

4.4 Coal-Based Applications of the Prototype Drill String Radar Tool ........... 76

4.5 Conclusions ............................................................ 77

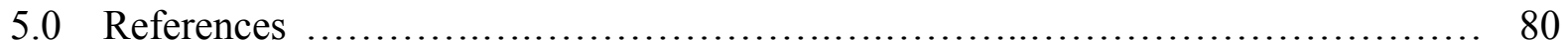




\section{$\underline{\text { List of Graphical Materials }}$}

$\underline{\text { Figure }}$

2-1 Characteristic impedance $\mathrm{Z}_{0}$ of a transmission line formed by a water-filled, Page (2.375-inch) diameter coiled-tubing with an inserted 0.25 -inch diameter slickline .. 4

2-2 Block diagram of synchronous detection of frequency-shift keying signals ........ 4

2-3 Downhole components of the first-generation prototype DTS $\ldots \ldots \ldots \ldots \ldots \ldots \ldots \ldots . . . \ldots$

2-4 Surface receiver setup of the first-generation prototype DTS at Snyder, Texas $\quad \ldots . . . \quad 7$

2-5 Field test of the first-generation prototype DTS at the Deer Creek Coal Mine

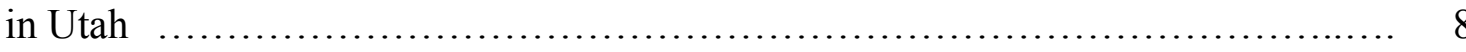

2-6 Components of the second-generation downhole-capable prototype DTS ......... 9

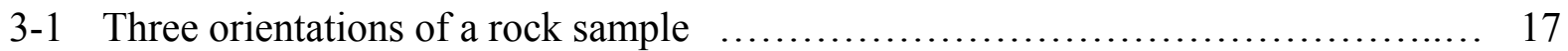

3-2 Range of electric conductivity and relative dielectric constant for natural media ... 17

3-3 Electrical properties of dry coal and roof shale samples from an underground

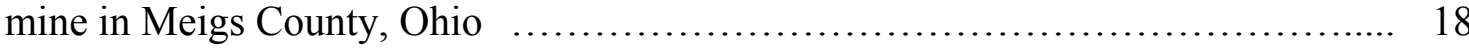

3-4 Agilent 16451B dielectric test fixture used for low-frequency testing of solid

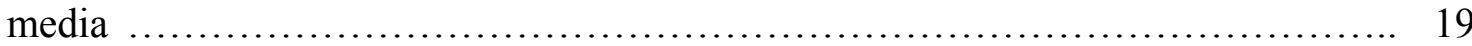

3-5 High-frequency instrumentation for measuring electrical properties of rocks $\ldots \ldots . .20$

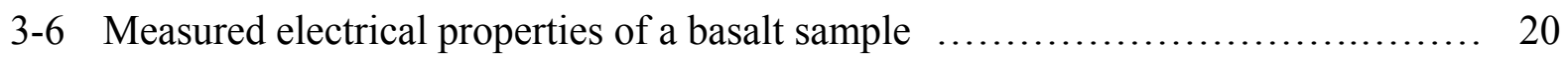

3-7 Measured electrical properties of a silty sandstone sample ..................... 21

3-8 Comparison of the EM material properties from the bedding plane samples of coal

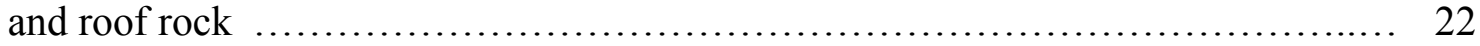

3-9 Electrical conductivity of sedimentary rock versus frequency $\ldots \ldots \ldots \ldots \ldots \ldots \ldots . \ldots . \ldots 23$

3-10 Attenuation rate and phase constant for a uniform plane propagating in a natural

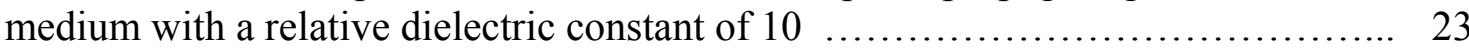

3-11 Skin depth and wavelength in a natural medium with a relative dielectric constant of 10

4-1 Vertical cross-section illustrating directional drilling in an oil-saturated sandstone

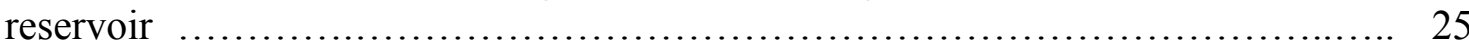

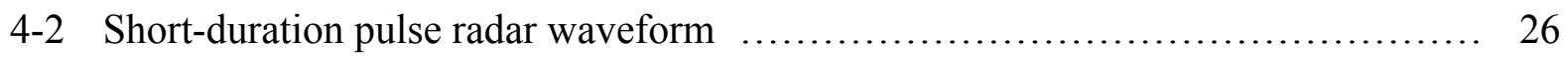

4-3 Time and frequency domain of a short-duration pulse radar ................... 27

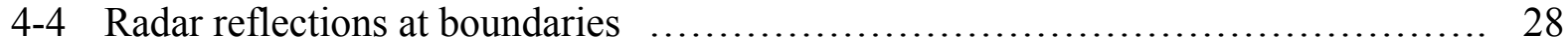

4-5 Electromagnetic wave energy flow in a drill string radar application $\ldots \ldots \ldots \ldots . . .30$

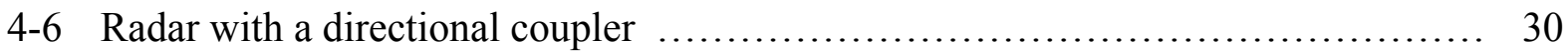

4-7 Functional diagram of the transmitted and received signals in FMCW and SFCW radar systems 
4-8 Stepping frequency and bandwidth required for the proper operation of an SFCW

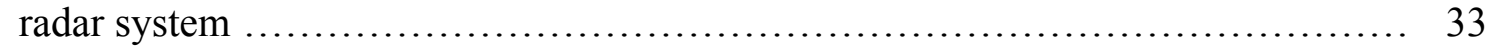

4-9 Resonant microstrip patch antenna (RMPA) ............................. 35

4-10 Impedance change with distance to the boundary rock $\ldots \ldots \ldots \ldots \ldots \ldots \ldots \ldots \ldots \ldots$

4-11 Near- and far-field measured data of a stepped-frequency, continuous-wave radar .. 36

4-12 Double-sideband, suppressed-carrier waveform ........................... 37

4-13 Software definable transceiver (SDT) radar electronics $\ldots \ldots \ldots \ldots \ldots \ldots \ldots \ldots \ldots . \ldots \ldots$

4-14 Top and bottom surfaces of the very low frequency and low frequency/medium

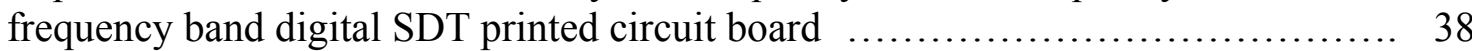

4-15 Block diagram of a software definable transceiver radar ....................... 39

4-16 Detailed functional block diagram of the software definable transceiver $\ldots . \ldots . \ldots .40$

4-17 Frequency chart for the software definable transceiver $\ldots \ldots \ldots \ldots \ldots \ldots \ldots \ldots \ldots . \ldots 1$

4-18 Surface electromagnetic gradiometer response over a subsurface void ............ 42

4-19 Prototype mid-seam guidance in a horizontal borehole in an oil reservoir ......... 43

4-20 Prototype mid-seam guidance tool antenna orientations ........................ 43

4-21 Construction features of the prototype mid-seam guidance tool for testing in a

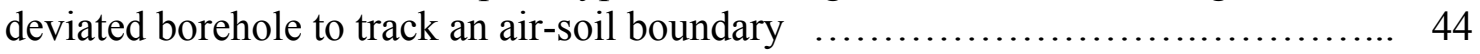

4-22 Location of the deviated test boreholes for tracking an air-soil boundary .......... 44

4-23 Reflection from antenna 1 in the deviated test borehole $\ldots \ldots \ldots \ldots \ldots \ldots \ldots \ldots \ldots . . \ldots 6$

4-24 Reflection from antenna 2 in the deviated test borehole $\ldots \ldots \ldots \ldots \ldots \ldots \ldots \ldots \ldots . \ldots 7$

4-25 Transmission loss between the two antennas in the deviated test borehole ......... 48

4-26 Measured transmission results with a transmitter-receiver pair of tuned loop antennas in the 11 -degree deviated borehole test site $\ldots \ldots \ldots \ldots \ldots \ldots \ldots \ldots \ldots \ldots . \ldots . \ldots \ldots$

4-27 Measured transmission results with a transmitter-receiver pair of tuned loop

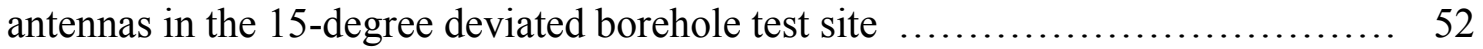

4-28 Mapping of a hydrocarbon reservoir with directional antenna patterns $\ldots \ldots \ldots \ldots .55$

4-29 Drill string radar navigation bottom hole assembly $\ldots \ldots \ldots \ldots \ldots \ldots \ldots \ldots \ldots \ldots . \ldots \ldots$

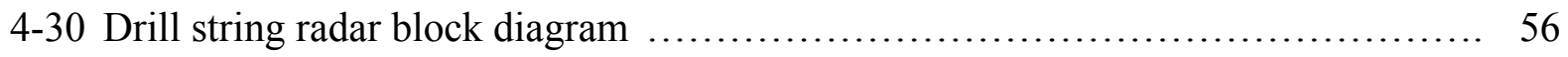

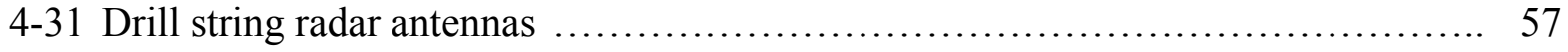

4-32 Major components of the initial drill string radar design $\ldots \ldots \ldots \ldots \ldots \ldots \ldots \ldots \ldots \ldots \ldots$

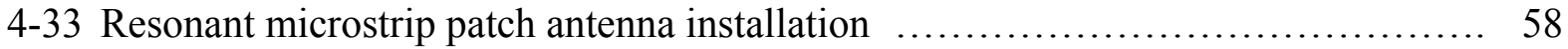

4-34 Stolar laboratory drill string radar borehole simulator $\ldots \ldots \ldots \ldots \ldots \ldots \ldots \ldots \ldots \ldots . \ldots$

4-35 Proof-of-concept field testing of the drill string radar antennas in a coal mine .... 59 
4-36 Conceptual design of a software definable transceiver drill string radar with

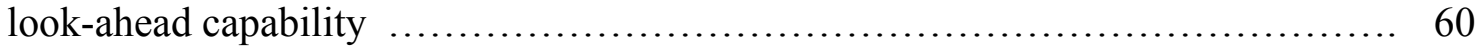

4-37 Plan view of the drill string radar prototype four-quadrant antenna pattern $\ldots \ldots \ldots .61$

4-38 Prototype continuous-wave drill string radar tool in a simulated vertical test setting

4-39 Polar suppression factor represented by near- and far-zone suppression superimposed on

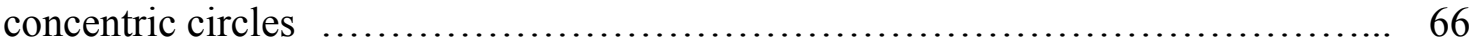

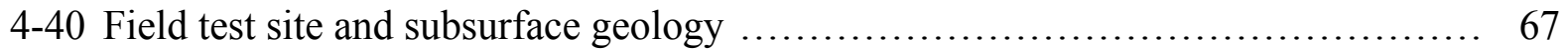

4-41 Prototype drill string radar tool prior to being lowered into the source borehole

4-42 Command and control of the prototype drill string radar tool being executed via a laptop computer

4-43 Computer screen shot of the surface graphical user interface for command and control of the prototype drill string radar tool

4-44 Computer screen shot of one page of raw and processed data

4-45 Collocated quadrature transmitting and receiving antenna array in the prototype drill string radar tool

4-46 Graphical presentation of the acquired receiver data 74

4-47 Data for the two separate sets of measurements taken at a depth of 180 feet 75

4-48 Naturally occurring undulations in coal seams caused by an overlying paleochannel

4-49 Sidetracks found in horizontal drilling in coal seams for coal bed methane production

4-50 Comparison of conventional horizontal drilling and radar-controlled drilling in a coal seam

4-51 Radar-assisted eagle track drilling in coal seams

4-52 Stolar Drill String Radar and Data Transmission System in a coiled tubing drilling application 


\section{List of Tabular Materials}

$\underline{\text { Table }}$

$\underline{\text { Page }}$

4-1 Maximum range and resolution of a stepped-frequency radar system with 33 


\section{$\underline{\text { List of Acronyms and Abbreviations }}$}

\begin{tabular}{|c|c|}
\hline $\mathrm{A} / \mathrm{D}$ & analog to digital \\
\hline $\mathrm{AGC}$ & automatic gain control \\
\hline AWG & American wire gauge \\
\hline BW & bandwidth \\
\hline CBM & coal bed methane \\
\hline CCA & circuit card assembly \\
\hline CMM & coal mine methane \\
\hline $\mathrm{CW}$ & continuous wave \\
\hline $\mathrm{D} / \mathrm{A}$ & digital to analog \\
\hline $\mathrm{DC}$ & direct current \\
\hline DDS & direct digital synthesizer \\
\hline DOE & Department of Energy \\
\hline DSBSC-AM & double-sideband, suppressed-carrier amplitude modulation \\
\hline DSR & drill string radar \\
\hline DTS & data transmission system \\
\hline EM & electromagnetic \\
\hline FMCW & frequency-modulated, continuous-wave \\
\hline FPGA & field-programmable gate array \\
\hline FSK & frequency-shift keying \\
\hline GIPP & Global Initiative for the Prevention of Proliferation \\
\hline GUI & graphical user interface \\
\hline HMD & horizontal magnetic dipole \\
\hline HP & Hewlett Packard \\
\hline $\mathrm{Hz}$ & Hertz \\
\hline I & in phase \\
\hline IAP & in-application programming \\
\hline I.D. & inside diameter \\
\hline $\mathrm{kHz}$ & kilohertz \\
\hline LF & low frequency \\
\hline $\mathrm{m}$ & meter \\
\hline $\mathrm{mA}$ & milliampere \\
\hline $\mathrm{MHz}$ & megahertz \\
\hline MF & medium frequency \\
\hline MSHA & Mine Safety and Health Administration \\
\hline MWD & measurement-while-drilling \\
\hline NASA & National Aeronautics and Space Administration \\
\hline NBFM & narrow-band frequency modulation \\
\hline O.D. & outside diameter \\
\hline PCB & printed circuit board \\
\hline PDA & personal digital assistant \\
\hline PA & power amplifier \\
\hline PLL & phase locked loop \\
\hline psi & pounds per square inch \\
\hline PVC & polyvinyl chloride \\
\hline
\end{tabular}




$\begin{array}{ll}\text { Q } & \text { quadrature } \\ \text { R\&D } & \text { research and development } \\ \text { RF } & \text { radio frequency } \\ \text { RFI } & \text { radio frequency interference } \\ \text { RX } & \text { receiver or receive } \\ \text { S } & \text { second } \\ \text { SDT } & \text { software definable transceiver } \\ \text { SFCW } & \text { stepped-frequency, continuous-wave } \\ \text { TD } & \text { total depth } \\ \text { TVD } & \text { true vertical depth } \\ \text { TX } & \text { transmitter or transmit } \\ \text { VLF } & \text { very low frequency } \\ \text { VMD } & \text { vertical magnetic dipole }\end{array}$




\section{$\underline{\text { Executive Summary }}$}

Under U.S. Department of Energy (DOE) Contract Number DE-FC26-04NT15477, Stolar Research Corporation (Stolar) has successfully developed pre-commercial prototypes of two technologies, the Stolar Data Transmission System (DTS) and the Stolar Drill String Radar (DSR), for application in coiled tubing drilling environments. These technologies are also applicable to conventional segmented pipe drilling.

The importance of these two technologies has been recognized nationally. For example, $R \& D$ Magazine selected Stolar's DSR and DTS technologies for R\&D 100 Awards in 2005 and 2006, respectively. These prestigious awards are given to designate the 100 most technologically significant products of the year.

The DTS provides real-time, half-duplex communications with downhole sensors for the oil and gas industry using coiled tubing, standard drill rod, or slickline as the transmission medium. In addition, the DTS is an integral part of the Stolar DSR system by providing two-way communications with drill string sensors for real-time measurement-while-drilling (MWD) applications. This capability facilitates geosteering in horizontal drilling regimes with extremely fast data rates (i.e., $\sim 2,400$ bits/second) and, thus, response times. This high data rate is a significant advance when compared to commercially available systems and allows continuous orientation of the drilling process in real-time MWD applications. The DTS is designed to meet a wide variety of downhole-to-surface data communications needs and can be easily configured to mate with conventional downhole sensors and drilling hardware.

The Stolar DTS has a number of important and distinguishing characteristics:

- provides two-way radio frequency data communication;

- inductively couples modulated (i.e., frequency-shift keying) radio waves $(\sim 100 \mathrm{kHz})$ to the skin of the drill pipe, coiled tubing, or slickline systems using specially designed antennas;

- uses natural waveguide properties of the hydrocarbon deposit;

- entire drill rod (i.e., coiled tubing or segmented pipe) and the immediate surrounding layers of rock are the data transmission waveguide;

- operates with a higher carrier signal and greater bandwidth than most common competitive systems, allowing higher data rates (i.e., $~ 3,000$ times for coiled tubing and $\sim 300$ times for segmented pipe); and

- operates regardless of hydraulic pressures, acoustics, or proximity to, or contact with, the side of the wellbore or casing.

The design of the DSR provides geosteering functionality for the oil and gas industry using coiled tubing or standard drill rod. The DSR is designed to meet the requirements of a wide variety of MWD techniques and the bottom hole assembly can be easily configured to mate with standard drilling hardware. Additional work to make the DSR design more rugged to withstand the wide range of downhole conditions encountered in both coiled tubing and conventional drilling is ongoing under an agreement with a major international oilfield service company that will assist in the eventual commercialization of the Stolar DSR product line. 
The Stolar DSR design has a number of important and distinguishing characteristics:

- propagates low- and medium frequency radio waves through sedimentary rock or hydrocarbon layers;

- operating frequency and dynamic range depend on the electrical properties of the host rock;

- measures dielectric constant and distance to boundary rock with a design range of approximately 40 feet;

- determines sedimentary rock characteristics and thickness of a hydrocarbon reservoir

- operates as a true radar device;

- suppression algorithms eliminate clutter from near-field or borehole wall reflections while enhancing reflections of more distant targets;

- low-frequency phase-modulated compound antennas suppress back lobes and generate directional electromagnetic fields; and

- electromagnetic fields are steerable electronically allowing the DSR to look up and down without rotating the drill rod.

Integration of the two Stolar technologies results in a system that has a wide variety of applications beyond coiled tubing drilling, including:

- many geological and geotechnical drilling applications, including exploration drilling;

- conventional oil and gas reservoir production drilling, particularly horizontal drilling;

- horizontal coal bed methane (CBM) drilling, especially in thin seams; and

- coal mine methane (CMM) drainage holes, particularly in long wall mining environments. 


\subsection{Introduction}

\subsection{Objectives and Major Accomplishments}

The overall objectives of the Radar Navigation and Radio Data Transmission for Microhole Coiled Tubing Bottom Hole Assemblies project conducted by Stolar Research Corporation (Stolar) were to design, fabricate, and test two advanced technologies of importance to the future growth of the U.S. oil and gas industry: (i) real-time measurement-while-drilling (MWD) for guidance and navigation of coiled tubing drilling in hydrocarbon reservoirs and (ii) two-way inductive radio data transmission on coiled tubing or via an insulated slickline (i.e., smalldiameter solid wire similar to piano wire) fed inside the coiled tubing. The operating specifications for these technologies were to be compatible with 3.5-inch boreholes drilled to a true vertical depth (TVD) of 5,000 feet.

These two technologies were developed into pre-commercial prototypes and tested in simulated coiled tubing drilling conditions. The results of this advanced development work should be an important step in the expanded commercialization of advanced coiled tubing microhole drilling equipment for use in U.S. hydrocarbon reservoirs. In certain applications, the use of coiled tubing drilling technology provides significant cost and environmental advantages when compared to the use conventional segmented, large-diameter drill pipe, particularly in shallow (i.e., $<5,000$ foot) in-fill drilling situations.

As a result of the work performed as part of this project, $R \& D$ Magazine selected Stolar's Drill String Radar and Data Transmission System for R\&D 100 Awards in 2005 and 2006, respectively. These prestigious awards are given to designate the 100 most technologically significant products of the year. Furthermore, the work performed under this project provided the technical foundation for the establishment of a joint development agreement with the goal of further development and commercialization of the basic drill string radar technology for use initially in heavy oil recovery applications. Heavy oil resources represent an extremely important source of expanded oil production in North America. The parties to this agreement are Stolar and one of the major U.S.-based international oilfield service supply companies.

\subsection{Organization of this Report}

This report is organized into five major sections. Section 1.0 summarizes the objectives of the sponsored R\&D project and the major accomplishments that have resulted from the work that was conducted. The Stolar Data Transmission System (DTS) that was developed as part of the $\mathrm{R} \& \mathrm{D}$ project is the subject of Section 2.0. Electrical properties of rocks are discussed in Section 3.0. These properties play an important role in subsurface electromagnetic wave propagation, which is the basis of the Stolar Drill String Radar (DSR) technology. Section 4.0 summarizes the theory, hardware design work, and prototype field testing that was conducted on the DSR technology. Cited technical references are compiled in Section 5.0. 


\subsection{Data Transmission System}

The primary function of Stolar's Data Transmission System (DTS) is to provide two-way radio frequency data communications on coiled tubing drilling systems in an equipment package that can be integrated seamlessly into existing coiled tubing drilling equipment. This basic technical capability can also be easily modified for use on standard, segmented drill pipe. The DTS system transmits real-time data between standard downhole sensors and surface communications equipment. Real-time data transmission is an integral part of any advanced measurement-whiledrilling (MWD) technology.

Several commercial systems (e.g., extremely low frequency through-the-earth electromagnetic systems, electro-hydraulic or mud-pulse systems, electro-mechanical or acoustic systems, specialized drill pipe with embedded conductive cables, and standard large-diameter wireline systems widely used in well logging applications) are currently available to accomplish downhole-to-surface communications. In addition, slickline-based well logging tools record data in memory downhole, which are then downloaded for processing on the surface when the tool has been removed from the wellbore. However, all of these systems employ technical approaches different than the Stolar DTS, which accomplishes data transmission through modulated radio waves inductively coupled to the external skin of the drill pipe using loop antennas.

The Stolar DTS communications mode permits a higher frequency carrier with a greater bandwidth. Specifically, the DTS is capable of operating at 2,400 bits/second, which is an extremely fast data rate compared to most conventional downhole communications systems. Higher data rates allow more information to be transmitted to the surface. Low- and mediumfrequency, inductive emergency communications systems have been developed for use in underground mining applications, however [1]. The transmission networks for these systems have included metallic pipe, rails, wire rope, and conveyor belt structures.

\subsection{Theoretical Considerations}

Theoretical formulations for calculating attenuation rates for propagating electromagnetic (EM) waves in subsurface media have been developed by Wait and Hill [2]. The bifilar transmission modes exhibit significantly lower attenuation rates than monofilar modes. Bifilar modes require two conductors for EM wave propagation, while monofilar modes require only a single conductor. Wait and Hill [2] investigated EM propagation for data transmission on a vertical drill string, which is directly applicable to the induction of EM waves on coiled tubing. Coiled tubing, without the presence of any other conductor, supports the monofilar mode. An insulated slickline fed into coiled tubing supports the bifilar mode of EM wave propagation.

Low- and medium-frequency attenuation rates were determined for the bifilar and monofilar transmission modes for coiled tubing transmission networks. Attenuation rates of less than $1 \mathrm{~dB}$ per 1,000 feet of slickline and approximately $2 \mathrm{~dB}$ per 100 feet of coiled tubing for the bifilar and monofilar modes, respectively. 
Because coiled tubing is a hollow metal structure, the inside diameter will act as a cylindrical waveguide [3] that can transmit EM waves whose frequencies are above the cutoff frequency given by

$$
f_{c}=\frac{1.841 v}{2 \pi a}
$$

where $a=$ radius of the coiled tubing cross section,

$v=$ the speed of the EM wave $=\mathrm{c} / \sqrt{\varepsilon_{r}}$ for non-magnetic media filling the waveguide,

$\mathrm{c}=$ the speed of light in a vacuum $\left(3 \times 10^{8} \mathrm{~m} / \mathrm{s}\right)$, and

$\varepsilon_{r}=$ the relative dielectric constant of the media filling the coiled tubing.

Using Equation (2-1), a water-filled ( $\varepsilon_{r}=80$ ), 2.375-inch pipe will have a cutoff frequency of $325 \mathrm{MHz}$. Such a high-frequency signal will not propagate far inside coiled tubing before being attenuated greatly. Thus, the inside of the coiled tubing would act as a high-pass filter when radio waves are propagating inside.

Placing an insulated slickline inside coiled tubing is equivalent to having a coaxial transmission network with the slickline as the center conductor and the coiled tubing as the outer conductor shield. A coaxial transmission network is a low-loss and low-pass medium operating in the bifilar mode as long as the filling medium is not too lossy and the center conductor does not become shorted against the sidewalls. The latter situation is the reason for insulating the slickline by applying a dielectric coating.

The characteristic impedance, $\mathrm{Z}_{0}$, of a coaxial line is given by

$$
Z_{0}=60 \ln \left(x+\sqrt{x^{2}-1}\right) / \sqrt{\varepsilon_{r}},
$$

where

$$
x=\frac{d^{2}+D^{2}-4 O^{2}}{2 d D}
$$

and where $d=$ the diameter of the inner conductor (slickline),

$\mathrm{D}=$ the inner diameter of the outer conductor (coiled tubing),

$\mathrm{O}=$ the offset between the centerline of the two conductors, and

$\varepsilon_{r}=$ the relative dielectric constant of the filling medium.

The characteristic impedance as a function of the offset between the conductors is shown in Figure 2-1. The inner conductor (slickline) would short against the outer conductor when $\mathrm{x}=1$ as given by Equation (2-3) or when the offset is exactly equal to $\mathrm{D} / 2-\mathrm{d} / 2$. 


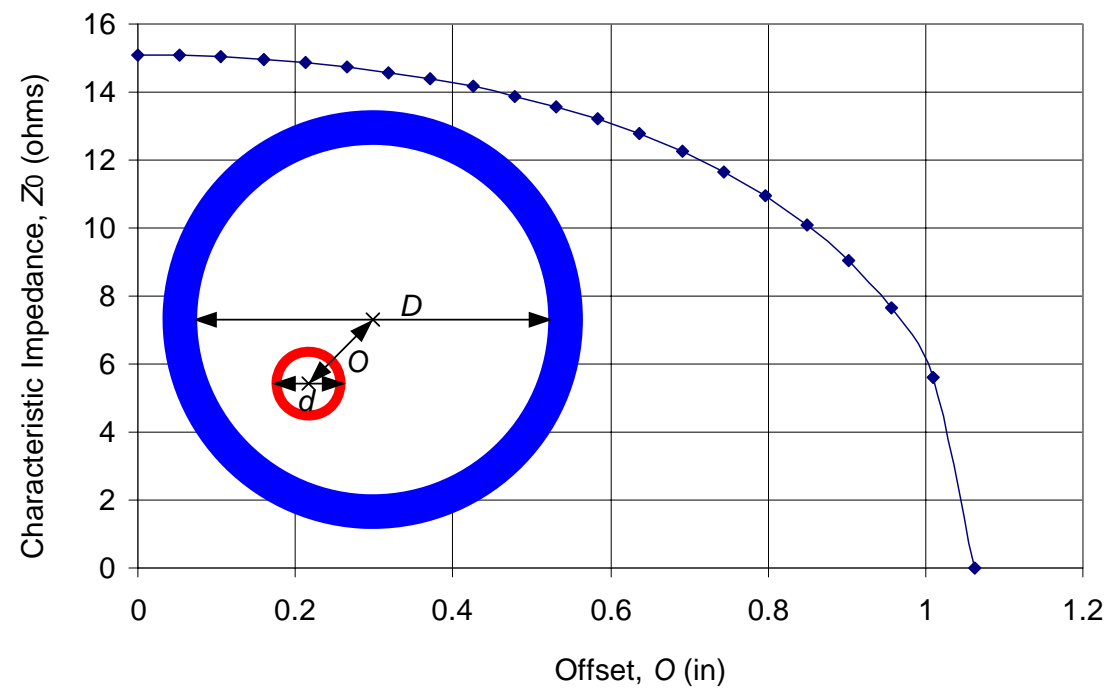

Figure 2-1. Characteristic impedance $Z_{0}$ of a transmission line formed by a water-filled, (2.375-inch) diameter coiled-tubing with an inserted 0.25-inch diameter slickline. When the slickline touches the coiled-tubing, $Z_{0}=0$.

The inductively coupled transmission network allows the propagation of low-frequency waves on the outer surface of the coiled tubing without the presence of an insulated slickline. The entire coiled tubing and the immediate surrounding rock, in contrast to the layered earth, becomes the data transmission network. Operation bandwidth, noise, and modulation procedure affect the ultimate performance of the data transmission network.

\subsection{First-Generation Prototype Data Transmission System}

\subsubsection{Experimental Design of the First-Generation Prototype}

The basic design of the first-generation DTS employed frequency-shift keying (FSK) modulation [4] of 91.5-kHz signals. This center frequency (i.e., $91.5 \mathrm{kHz}$ ) accommodates the losses in the transmission channel with reasonable system size and power requirements. The EM waves are inductively coupled to the outer surface of the coiled tubing by a loop antenna. A commercial downhole navigation package was used to provide signals for the testing program.

To represent the binary signals in the FSK modulation technique, two base-band sine waves with radian frequencies of $\omega_{1}$ and $\omega_{2}$ are transmitted. A block diagram of the synchronous detection, which is the reverse of the original modulation process at the transmitter, of the FSK signals is shown in Figure 2-2, where $f_{C}(t)$ is the signal captured by the receiver antenna. In the absence of noise, the bandwidth is the limiting factor in determining the transmission rate. For example, in the first-generation prototype data transmission system, the transmission rate was $2,400 \mathrm{bits} / \mathrm{s}$ due to the $3-\mathrm{kHz}$ bandwidth of the system. The first-generation prototype was configured for testing in a pre-drilled hole only and consists of hardware and radio communications software with a LabView ${ }^{1}$ graphical user interface (GUI).

\footnotetext{
${ }^{1}$ LabView is a trademark of National Instruments Corporation, Austin, Texas.
} 
The hardware of the first-generation prototype DTS comprises downhole and surface hardware. The downhole hardware consisted of a navigation package with magnetometers and accelerometers, electronics, an antenna, and a battery pack all connected as a single unit (see Figure 2-3). The surface hardware consisted of electronics/battery unit, with a tripod mounted antenna, and a laptop interface.

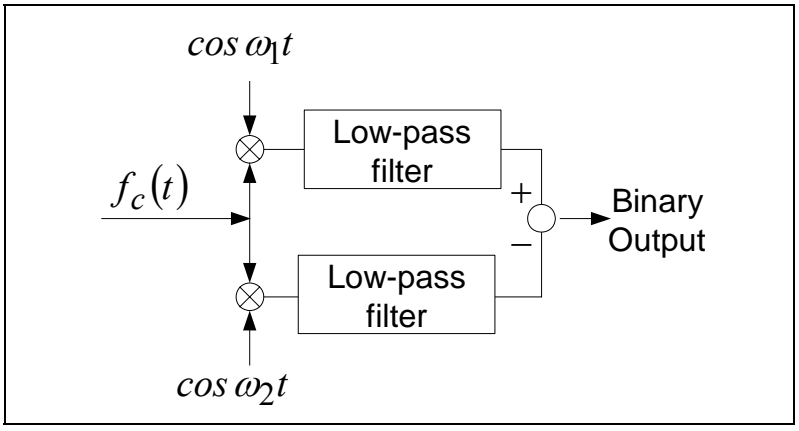

Figure 2-2. Block diagram of synchronous detection of frequency-shift keying signals.

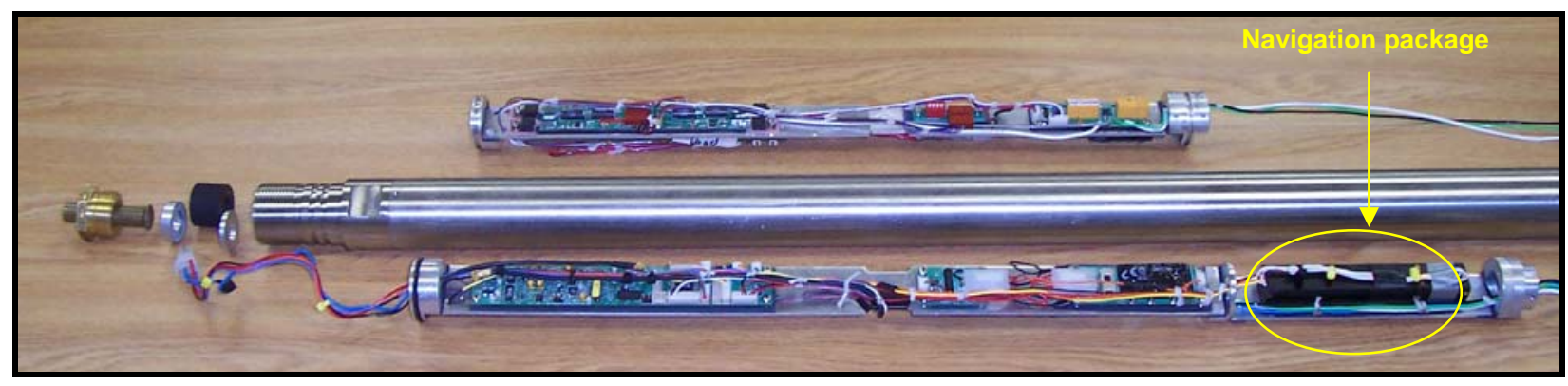

(a)

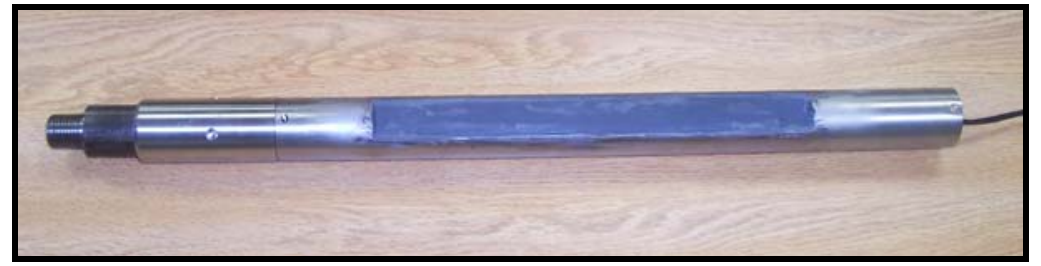

(b)

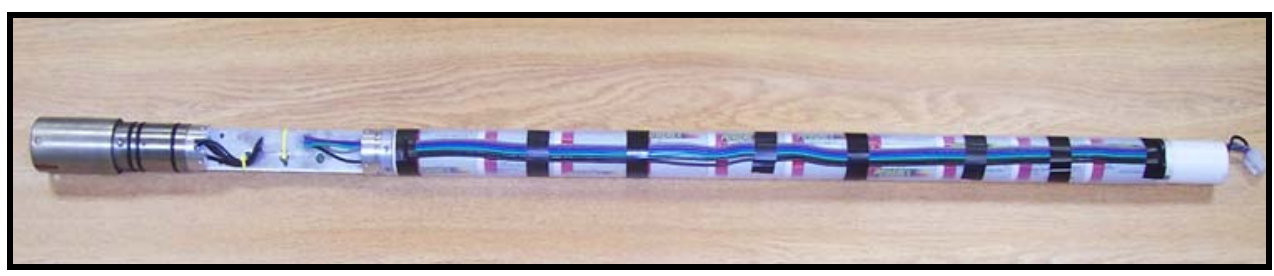

(c)

Figure 2-3. Downhole components of the first-generation prototype DTS: (a) electronics with the navigation package, (b) a longitudinally wrapped, potted, loop antenna, and (c) the battery pack. 
The antenna geometry is a major component of the data communications design. Multiple geometries of ferrite-loaded and other loop antennas were considered for the downhole antennas. Ferrite-loaded antennas were eliminated due to their core saturation in the presence of highmagnitude fields. Two downhole antenna designs were selected for testing: (i) a loop antenna longitudinally wound on a rectangular plastic blank and (ii) a toroidal antenna.

The antennas were configured for two basic mounting/signal coupling configurations: slickline and coiled tubing. In the slickline configuration, the slickline cable was electrically isolated from the case of the downhole tool. The slickline cable was brought into the tool on a center conductor and electrically mated to the antenna's copper mounting bracket. The antenna coupled the signal to the mounting bracket and onto the slickline. In the coiled-tubing mounting configuration, the antenna was embedded in the tool casing and coupled directly to the outside diameter surface (skin) of the downhole tool.

The first-generation prototype DTS was designed for one-way data transmission, downhole to surface. Two-way data transmission had been accomplished in other configurations of the same hardware, so achieving two-way transmission was possible from a functional standpoint, provided suitable signal-to-noise ratios at a usable signal propagation distance could be achieved.

A LabView graphical user interface (GUI) was developed on a laptop computer to display realtime data from the downhole tool. LabView is a developmental tool that facilitates prototype development. The GUI displays three-axis tool orientation, which is compiled from the downhole navigation data.

At the surface receiver, the automatic gain control (AGC) level indicates how much the receiver is amplifying the received signal, that is, the AGC indicates the magnitude and quality of the signal. High AGC levels mean the receiver is working hard to detect a weak signal, thus the system is getting close to the effective communication limit.

\subsubsection{Experimental Results for the First-Generation Prototype}

The DTS antennas were electromechanically coupled to a stainless steel cable, simulating coiled tubing and slickline because the cable was easier to handle and could be used under a wider range of scenarios. Stainless steel (multiple alloys), beryllium copper, "ToughMet" ${ }^{\circledR}$ (i.e., a copper-nickel alloy), and titanium were evaluated as potential materials used to house the antenna to maximize antenna performance. The antenna's performance was unaffected when housed in beryllium copper. Other housing materials, especially magnetic material, adversely affected the antenna's performance. Thus, these tests of the first-generation prototype suggested that beryllium copper is the preferred housing material.

The first generation prototype was designed to test the feasibility of the data transmission concept in a pre-drilled hole only, without consideration to the physical requirements of interfacing with coiled tubing or segmented drill pipe. The first-generation DTS hardware and software of the data communications system were initially functionally tested in Stolar's laboratory. For an initial field test of signal propagation distance on a slickline, a 1,700-foot 
cable was placed directly on the ground surface outside of Stolar's laboratory. Communication was successfully achieved between the transmitter and the receiver units, which were located at the opposite ends of the cable. There was sufficient range in the AGC of the receiver to allow for greater transmission distances. In addition, a test was conducted using a 600-foot section of 2.875-inch O.D. drill pipe laid in a field. The results were similar to those of the initial 1,700foot test using a cable.

A second field test was conducted in Snyder, Texas, using a water-filled test well (see Figure 24). The well was 340 feet deep, with a water level 20 feet below the surface. Surface casing was cemented into the top 10 feet of the well and casing was cemented in the well to the total depth. The ambient noise level next to the surface receiver was high because of the proximity of electrical power lines. The prototype DTS worked until the antenna assembly became submerged in water. Once the antenna section was submerged, the signal was attenuated to ambient noise floor levels, which prevented the signal to be measured at the surface receiver. Successful communication resumed once the antenna section was withdrawn from the water.

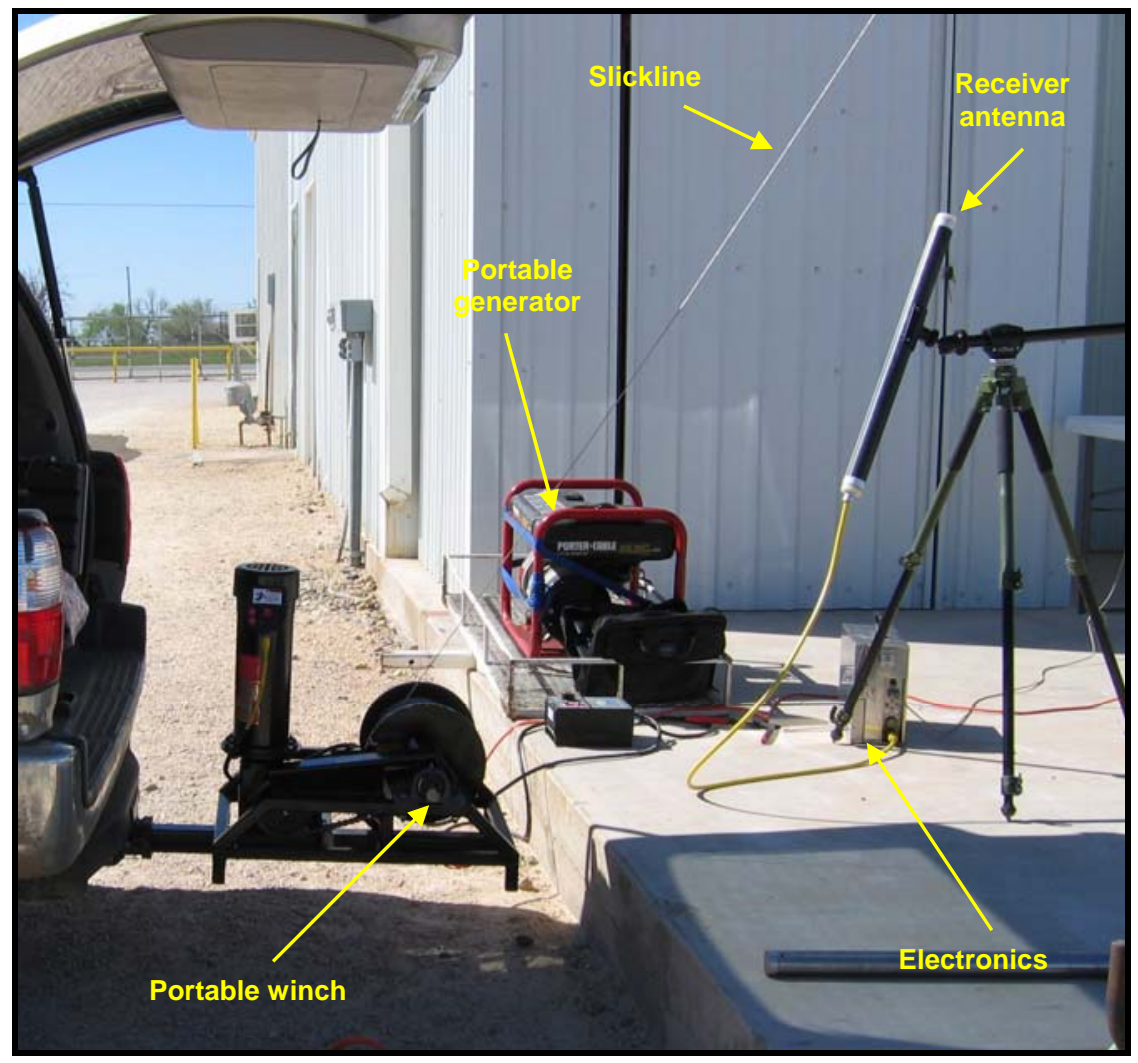

Figure 2-4. Surface receiver setup of the first-generation prototype DTS at Snyder, Texas.

Subsequent to the Texas field test, test fixtures for laboratory testing were built to emulate test conditions experienced in the 340-foot water-filled test well. The laboratory test results did not emulate the Texas test well results. The transmission did not fail in Stolar's laboratory tests when the antenna section was submerged in water as in the Texas test. Effective transmission was observed during the entire test. Thus, the high ambient noise level was speculated to be the cause of the communications failure in the Texas test. 
A third field test of the first-generation prototype DTS was conducted in a horizontally drilled hole in the Deer Creek Coal Mine in Utah (see Figure 2-5). This test platform provided an inexpensive and readily available site in which to conduct initial tests. The 3,000-foot hole was open and uncased beyond an initial collar.

The prototype DTS was pumped inside the test hole inside a 500-foot polyvinyl chloride (PVC) casing. At the end of the 500-foot mark, the DTS assembly was ejected by water pressure out of the PVC casing and into the uncased portion of the hole. The PVC casing was then removed from the hole, leaving the prototype DTS in the hole. The hole had a constant flow of water out of the annulus at a rate estimated to be between 20 and 30 gallons/minute. The transmission cable and downhole assembly were in direct contact with the coal formation and were immersed in water, which provided a harsh environment for signal propagation.

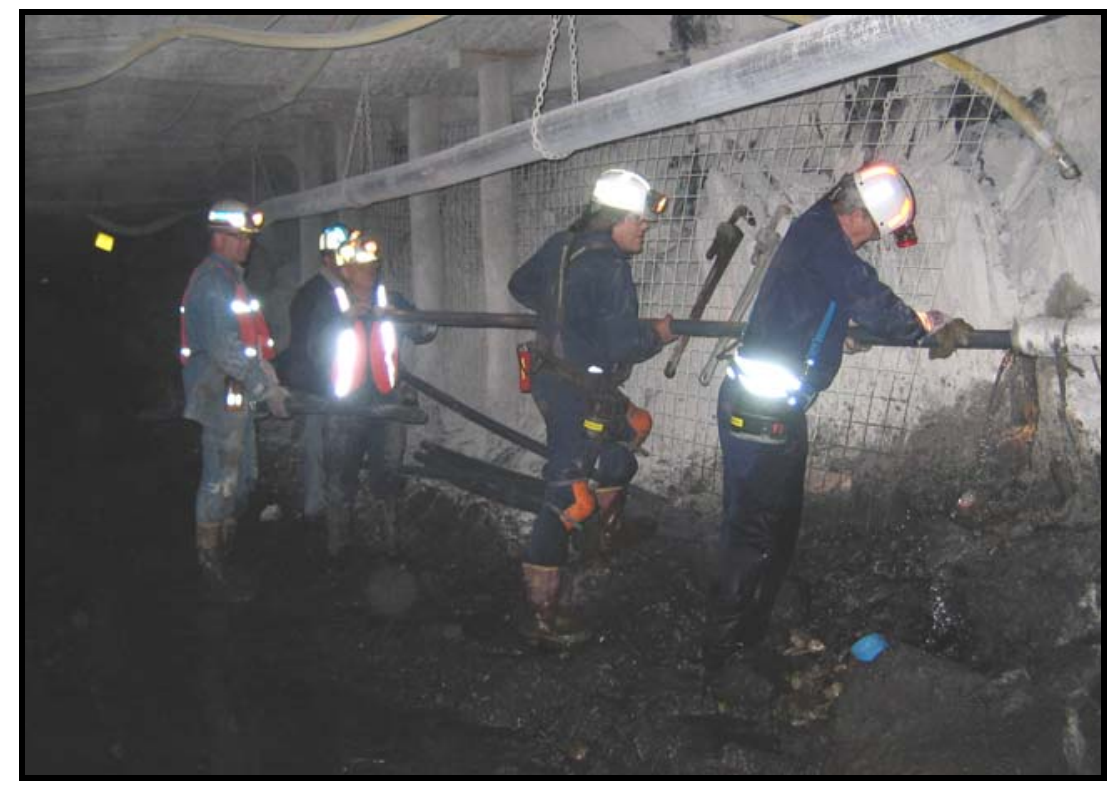

Figure 2-5. Field test of the first-generation prototype DTS at the Deer Creek Coal Mine in Utah.

Excellent communication was established between the downhole assembly and surface receiver, with an average signal-to-noise ratio of $30 \mathrm{~dB}$. Communication was maintained as the assembly was pulled through the 500 feet of hole back to the annulus and orientation data were successfully transmitted. As in the Texas field test, the operational characteristics of the prototype DTS were vulnerable to ambient noise levels. The average noise floor was $-90 \mathrm{dBm}$; however, the noise floor was $-60 \mathrm{dBm}$ when the electric winch for the cable spool was operating and generating radio frequency interference (RFI). Future designs of the prototype DTS must incorporate RFI noise shielding to achieve acceptable signal-to-noise ratios.

\subsection{Experimental Design of the Second-Generation Prototype Data Transmission System}

The basic design of the second-generation prototype DTS continued to employ frequency-shift keying (FSK) modulation of $91.5-\mathrm{kHz}$ signals. This center frequency accommodates the losses in the transmission channel with reasonable system size and power requirements. The EM 
waves are inductively coupled to the coiled tubing by a toroidal antenna in the second-generation prototype. A commercial downhole navigation package was used in the testing program.

The downhole hardware of the second-generation prototype DTS is shown in Figure 2-6. All of the electronics circuits are on mounted on low-profile, 1 -inch printed circuit boards.

(a)

(b)

(c)

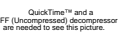

(d)

Figure 2-6. Components of the second-generation downhole-capable prototype DTS: (a) toroidal antenna with the protecting sleeve removed, (b) toroidal antenna assembly with protective sleeve installed (c) electronics assembly with navigation package, and (d) electronics assembly with hardened housing.

The second-generation prototype DTS was focused on designing a more robust pre-production prototype capable of data communications under actual drilling conditions. This design effort addressed issues such as assembly strength, fluid flow, abrasion resistance, integrated power 
production and distribution, user and maintenance friendliness, modular design, upgradeability, scalable design, antenna accuracy and optimization, vibration dampening, durability, economy, and radio frequency interference (RFI) reduction.

\subsubsection{Battery Pack}

The battery pack was designed to provide backup power to a downhole generator. The design utilizes $10 \mathrm{C}$-size, 1.2-volt, nickel metal hydride cells connected in series, which are rechargeable up to 1,000 cycles. The battery pack contains system power integration circuitry, allowing either a downhole generator or batteries to operate the DTS, and provides over-current protection. The battery pack is capable of powering short-term system use (i.e., 5 ampere hours at 12 volts or one to two hours of operating time under full system load). This backup capability allows for operation when a downhole generator is not operating because drilling fluid is not being pumped down the drill pipe. This backup power source also provides for the power up and functional testing prior to inserting the DTS into a borehole.

With planned future upgrades to the DTS, the battery pack should be capable of longer term power availability (i.e., 50 hours) under a power-saving system standby mode. Incorporating the hardware for this desirable feature into the DTS now saves development time and facilitates the development of system software options that enable a standby mode when the DTS is not being used. Examples of this situation include "tripping" the DTS in or out of a well and during suspended drilling operations.

The battery pack also contains circuitry for charging the battery by a downhole generator. The charging current is limited (i.e., 100 to $200 \mathrm{~mA}$ ) so that the battery pack is trickle charged, which prevents a discharged battery from consuming the available current produced by the generator. As such, a majority of the current would be available to power the DTS or supply other downhole power needs. There is a design tradeoff between quickly charging the battery and operating the DTS when the battery is charging. During most operating conditions, the DTS will operate on downhole generated power so that the battery will not be substantially discharged. The battery pack is also designed to be chargeable from an external source.

The battery pack design includes a provision for a magnetically activated on/off switch. The switch is located inside the housing, but is operated by a handheld magnet from outside the housing, which enables an operator to turn the DTS "on" or "off" without accessing the inside of the sealed housing. The battery pack is also equipped with an intermittent audible alarm when the system is turned on. A piezoelectric device emits a $3.9-\mathrm{kHz}$ tone, rated at $85 \mathrm{~dB}$ at two feet in air, loud enough to be heard outside the housing even when the housing is sealed. A three-second beep is emitted upon power-up, followed a one-second beep once every 60 seconds as long as power is applied, which gives the operator an audible indication that power is applied. This functionality is important for three reasons. First, during the initial power-up, the alarm gives a positive indication that power is applied, especially in the event that data transmission has not yet been established. Secondly, the absence of a beep gives positive indication that power is removed. Thirdly, when the DTS equipment is in storage, as in a warehouse, the audible alarm alerts a user that the DTS power has been left on. 


\subsubsection{Electronics}

The physical layout of the DTS electronics was reconfigured to fit in a housing similar to that of the battery pack. The design focused on efficient use of space, which is important to keep the overall size of the DTS to a minimum, and eliminates circuit card assemblies (CCAs) not required for the second-generation prototype. A geometry study of all system CCAs formed the basis for designing the new system layout. The study determined the required clearance to fit a suite of rectangular CCAs inside a narrower diameter housing. Furthermore, a modular design is used to enhance the assembly's stiffness and to facilitate a rapid reconfiguration for component upgrades and integration with other downhole systems. Particular attention was given to ensure ease of maintenance and assembly. The open layout provides access to all components and test points. Either whole modules or individual CCAs can be replaced.

The second-generation prototype design also concentrated on incorporating a layer of internal (to the housing) shock and vibration dampening to help isolate the DTS from the vibration encountered during a drilling operation. The shorter backbones, or chassis, on which the electronics are mounted increase stiffness of the assembly and inherently limit the possible vibration amplitude. This internal layer of shock dampening has limited travel amplitude and is not designed as the primary dampener. Instead, the internal dampening complements a dampener mounted external to the housing. The internal dampener affords some protection if the housing is dropped during handling and provides for three-axis dampening. The $\mathrm{x}$ - and $\mathrm{y}$-axis dampening is accomplished using large O-rings mounted between the electronic assembly and the inside of the housing. The O-rings are lubricated with silicon grease and are rated to operate in the temperature range of -40 to more than $400^{\circ} \mathrm{F}$. The O-rings are slightly compressed (i.e., $\sim 0.004$ in) when the assembly is inserted into the housing. The reserve elasticity of O-rings provides the dampening over a range of 0.020 inch. The O-ring scheme allows the entire assembly to move along the z-axis, which allows a z-axis dampener to be incorporated into an advanced design.

The reconfigured electronics housing design improved the ground isolation of the secondgeneration prototype DTS. The ground isolation in the first-generation prototype DTS was problematic by being structurally weak and prone to breaking. The updated design fully isolates the electronics ground from the housing, which is important to achieving efficient coupling of the DTS signal to coiled tubing.

\subsubsection{Antennas}

Antenna geometry is a major design consideration in the DTS. Based on the results obtained with the first-generation prototype DTS, a toroidal antenna configuration was selected for the second-generation prototype DTS. The electromagnetic fields of a toroidal antenna are on the same planes as those induced on the coiled tubing, which is a key to coupling efficiency. Greater contact path than other antenna geometries is possible with a toroidal antenna design. The magnetic field does not extend beyond the limits of the coil, thus making the antenna immune to near-field disturbances from objects outside of the toroid. Without this immunity, the antenna could de-tune in different environments. The EM transmission signal couples to a conductor that passes through the center of the toroid. 
Mechanical design factors, such as strength, concentration of stress, and flow of fluid, are simplified as a result of using a toroidal antenna. The coil is longitudinally wrapped around a non-ferrous, non-conductive core. For the second-generation prototype DTS, nylon 6/6 was used for the core. The core design is easily adapted to higher temperature plastics to facilitate higher temperature applications. All physical and some electronic parameters of the toroidal antenna are scaleable to design requirements.

In the second DTS prototype, the antenna is mounted in a robust fixture the same diameter as the drill pipe. In addition to housing the antenna, the fixture functions as another segment of drill pipe in the drill string. The signal is inductively coupled to the skin of the fixture and, in turn, electromechanically coupled to the skin of the drill pipe.

Based on the results of the first-generation prototype DTS work, a beryllium copper alloy, Brush Alloy 25, was the material of choice for housing the antennas. This alloy is very strong, with a tensile strength greater than 200,000 psi when heat treated, which allows smaller component thicknesses to achieve equal or greater strength compared to other candidate materials. Beryllium copper is highly conductive, within 15 to $20 \%$ of the International Annealed Copper Standard, which is four times more conductive than 304 stainless steel. High conductivity values increase the coupling efficiency of the antenna. Beryllium copper possesses very low relative magnetic permeability, between 0.997 and 1.003, which is near perfect transparency to slowly varying magnetic fields. Thus, the housing material will not affect the performance of the magnetometers inside a navigational package. In addition, the magnetic permeability of beryllium copper is unaffected by cold working in contrast to other nonmagnetic alloys, some of which become magnetically "hot" during machining, forming, or rigorous service. Beryllium copper is non-sparking and non-galling, which translates into good wear resistance, allowing contact with other materials with a minimum of friction and surface damage. In spite of these advantageous properties, beryllium copper is expensive and requires a specially equipped machine shop to handle the potential toxicity of the dust generated during machining operations.

Based on cost considerations and ease of handling, the first mockup of the second-generation prototype DTS antenna fixture was fabricated using 304 stainless steel and carbon steel to test the fit and functionality. The mockup was based on a 2.75 -inch NQ drill pipe. This approach allowed the design to be tested, fitted, faults detected, and refinements made prior to purchasing the expensive beryllium copper material. The choice of NQ drill pipe was based on availability of drillers using NQ pipe that have partnered with Stolar on development projects in the past. The higher strength requirements of NQ pipe exceed the requirements of coiled tubing.

The second-generation prototype DTS antenna fixture design consists of four sections. The first and main section is the cylindrical mandrel, with dimensions scalable to future design requirements. The toroidal antenna slides over the mandrel; thus, the mandrel is the section to which the antenna's signal is initially coupled. The antenna coil is electrically insulated from direct contact with the metal mandrel by a Mylar transformer tape layer that is 0.01 inch thick. The tolerance of the antenna's inside diameter (I.D.) is 0.01 to 0.02 inch above the outside diameter (O.D.) of the mandrel. This tight tolerance requires silicon lubricant to slide the antenna onto the mandrel and is an essential design element of the antenna. As such, the antenna coil is compressed slightly, forcing the toroidal coil into a uniform and predictable shape, which 
ensures that the antenna meets design characteristics and maintains consistent stable performance. The tight tolerance also maximizes coupling efficiency by placing the coil as close as possible to the mandrel. The mandrel is through drilled down the center to allow flow of drilling fluid. The I.D. is variable to the scalable design requirements. For the NQ pipe, a $0.875-$ inch hole was used, which permits fluid flow of 75 gallons per minute at 600 psi. The design incorporates an internal tube through the center hole of the mandrel along its entire length to allow the routing of cables, which serves the antenna cables as well as cables to and from adjacent housings.

The second section is the protective sleeve (see Figure 2-6b), which slides over the antenna to protect the antenna from abrasion and fluid intrusion. This sleeve is a cylindrical tube made of 304 stainless steel with electrical insulators installed at the ends of the sleeve to prevent bucking currents from interfering with the signal induced on the drill pipe. The dimensions of the protective sleeve are scalable to design requirements. For the NQ pipe, the tolerance of the I.D. of the sleeve is 0.01 to 0.02 inch above the O.D. of the antenna coil, and functions to compress the coil in the same fashion as the mandrel.

The third and fourth sections of the second-generation prototype DTS antenna design consist of end fittings (i.e., two pieces, one for each end) that serve as anchor points for the mandrel and the protective sleeve. One fitting bolts to each end of the mandrel using five high-strength bolts in a five-point "star" pattern. The protective sleeve slides over a portion of each end fitting. The junction is grooved for O-rings to seal against fluid intrusion. The end fittings couple the antenna-housing fixture to the drill pipe. The fittings are threaded and are scalable to design requirements. The current thread is for NQ pipe. The end fittings also function as attachment points for the electronics housings. The electronics housings are connected to the end fittings using a modular interface fixture, which facilitates changing the design to support different electronics housings designs without changing the antenna housing fixture design.

The physical parameters and requirements of coiled tubing and segmented drill pipe presented limitations to the physical dimensions of the antenna. Considerations included the O.D. of the tubing or pipe, the required I.D. to permit flow of drilling fluid, the required wall thickness to meet strength requirements of the tubing or pipe, and the required material thickness for abrasion resistance.

\subsubsection{Antenna Performance Modeling}

To calculate and predict the performance and physical design parameters of the toroidal antenna, a computer model was developed as a design tool. This model was used to calculate the antenna dimensions, namely, coil length, inside and outside diameter, wall thickness, number of loop turns, and the spacing between turns. The formula for predicting the inductance of a toroidal antenna is

$$
L=0.0046 N^{2} \Lambda \log _{10} \frac{D 2}{D 1}
$$

where $L=$ the inductance in Henries,

$N=$ the number of turns on the coil, 
$\Lambda=$ the length of the coil in inches,

$D 2=$ the outside diameter of the coil in inches, and

$D 1=$ the inside diameter of the coil in inches.

The formula's coefficient (0.0046) is an empirically derived number, which is impacted by the ratio of $D 2$ to $D 1$. For the current antenna design, the applicable coefficient is 0.003758 .

As a design tool, the computer model uses electronic and some physical parameters as inputs, allowing the user to specify the limits of the design. The model then calculates the remaining physical design using these design limits. This approach allows the user to quickly see the effects of specific variables, what parameter the variable impacts, and serves as an indicator of the tolerances to which a variable must be controlled to ensure accuracy of the design. Ultimately, the model calculates tube core dimensions and displays the required dimensional tolerances. These dimensions and tolerances are then used to machine a plastic tube, which serves as a framework to wrap the coil.

Using the computer program, key design variables were isolated and controlled to improve the accuracy of empirical values verses predicted values to less than $1 \%$. Some of the key variables controlled are:

- Coil length. The length of the coil has a direct impact on impedance and inductance of the antenna.

- Coil O.D., I.D., and core wall thickness. The dimensions of the coil impact inductance and impedance (e.g., differences of only 0.01 inch can make a $10 \%$ difference in measured inductance and impedance). Controlling these variables required incorporating coil compression into the physical design of the antenna-mounting fixture to force the coil into a uniform and predictable shape.

- Spacing between antenna turns (i.e., N spacing). Tighter spacing for a given number of turns increases impedance and increases the potential for more turns overall, which also increases inductance and impedance. The combined impedance increase is nonlinear and is sensitive to changes in spacing of less than 0.001 inch. Physically, this tolerance is very difficult to control. The individual effect per turn is very small, but when combined over a large number of turns, the combined effect can be significant (e.g., 20 to $30 \%$ effect on overall impedance). Control of this variable is maintained by wrapping the coil to maximum turn density or near zero spacing between turns. The impedance per turn at specific turn densities was measured empirically.

- American wire gauge (AWG). A low-gauge (i.e., thick-diameter) wire reduces the impedance of the coil in two ways. First, thicker diameter wires inherently have lower resistance, as governed by the formula

$$
R=\frac{l}{\sigma S}
$$

where $\mathrm{R}=$ resistance,

$l=$ the length of the wire,

$\sigma=$ the conductivity of the wire, and

$S=$ the cross sectional area of the wire. 
Secondly, larger diameter wire reduces the number of turns possible in a given coil. The difference in diameter in the AWG standard is nonlinear. The effect on the overall coil impedance was determined empirically.

- Frequency. Increasing frequency decreases impedance. To analyze this phenomenon, calculations at discrete frequencies were made, specifically $91.5 \mathrm{kHz}$ (i.e., the original center frequency), 100, 110, 120, 130, 140, and $150 \mathrm{kHz}$. Empirical measurements of impedance per turn at specific turn densities and overall coil impedance were made for these frequencies. Since the amplitude of RFI tends to be greater at frequencies below $90 \mathrm{kHz}$, and the Deer Creek Mine and Snyder, Texas, tests highlighted the vulnerability of the first-generation prototype DTS to RFI, collecting these data supported the possibility of increasing the center frequency slightly.

- Impedance. The total circuit impedance verses the coil's impedance needs to be controlled.

\subsubsection{Radio Frequency Interference}

The field-testing of the first-generation prototype DTS indicated that RFI was a significant problem. Reduction of in-band RFI is a major factor in the performance of the DTS. DC Blocks were added to the antenna circuit, consisting of three large capacitors in series. One DC block was placed in series with each antenna lead. The three capacitors provide a three-layer component failure protection.

The use of DC Blocks complicates antenna tuning by placing a large capacitance in series with the antenna coil because the total series capacitance of the DC Blocks is greater than the amount required to tune the antenna. Two-way transmission capability requires the antenna to operate in both transmit and receive modes, that is, series tune (i.e., low impedance) for the transmit mode and parallel tune (i.e., high impedance) for the receive mode. Prior to adding the DC Blocks to the design, a single capacitance value, switched from parallel to series, could tune the antenna in both modes. With the DC Blocks installed in series, the values required to tune in parallel differed from the values needed in series, which required switching between two different tuning channels. The first-generation prototype DTS design could only switch a single channel. An existing tuning board was adapted to facilitate two tuning channels on a single tuning CCA. This design saves space and avoids a major redesign of the tuning scheme.

\subsubsection{Graphical User Interface}

The graphical user interface (GUI) for the surface operator is a personal digital assistant (PDA). Buttons on a PDA have been re-programmed to display custom graphics, received from measurement electronics, on a real-time basis. The PDA can operate either wirelessly or with an attached wire with minimal power consumption.

\subsection{Coal-Based Applications of the Prototype Data Transmission System}

Although the DTS has been designed to operate in shallow liquid or gaseous hydrocarbon deposits, key aspects of the DTS design have addressed the conditions experienced in coal deposits. In the latter case, the DTS could be deployed in either coal mine methane (CMM) or 
coal bed methane (CBM) settings. Production of CMM involves in-mine drilling in gaseous environments. Because of operational safety considerations, all electrical equipment for use in coal mines must be approved by the Mine Safety and Health and Administration (MSHA) with respect to being explosion proof, flame proof, and/or intrinsically safe. The key electrical components of the DTS have been designed to meet these MSHA standards. As such, the current design of the DTS can be applied in coal mining environments without the need for basic design modifications.

\subsection{Conclusions}

A pre-commercial prototype data transmission system (DTS) has been designed, fabricated, and tested in both laboratory and field settings. The DTS provides real-time, half-duplex communications with downhole sensors for the oil and gas industry using coiled tubing, standard drill rod, or slickline as the transmission medium. Furthermore, the DTS is an integral part of the Stolar Drill String Radar system by providing two-way communications with drill string sensors for real-time measurement-while-drilling (MWD) applications. The DTS is designed to meet a wide variety of downhole-to-surface data communications needs and can be easily configured to mate with conventional downhole sensors and drilling hardware.

The Stolar DTS has a number of important and distinguishing characteristics:

- provides two-way radio frequency data communication;

- inductively couples modulated (i.e., frequency-shift keying) radio waves ( $100 \mathrm{kHz})$ to the skin of the drill pipe, coiled tubing, or slickline systems using specially designed antennas;

- uses natural waveguide properties of the hydrocarbon deposit;

- entire drill rod (i.e., coiled tubing or segmented pipe) and the immediate surrounding layers of rock are the data transmission waveguide;

- operates with a higher carrier signal and greater bandwidth than most common competitive systems, allowing higher data rates (i.e., $~ 3,000$ times for coiled tubing and $\sim 300$ times for segmented pipe); and

- operates regardless of hydraulic pressures, acoustics, or proximity to, or contact with, the side of the wellbore or casing. 


\subsection{Electrical Properties of Rocks}

The performance of radar-based subsurface navigation systems is heavily influenced by the electric properties of the medium in which the electromagnetic (EM) waves are propagated. Specifically, the ability to measure the distance to a boundary is controlled by knowledge of the dielectric constant of the surrounding rock, which affects the speed of travel of EM waves. A radar system detects the EM contrast between various media. Since the EM properties of rocks are frequency-dependent, a judicious choice in determining the operating frequency is paramount to the success of a radar system. For example, a radar system will not work if it encounters large losses (i.e., very low resistivity values) in its surrounding medium.

The electrical properties of rocks (e.g., dielectric constant, conductivity, and resistivity) depend on a number of parameters, including moisture content and petrographic character [5]. The electrical properties are also dependent on the relative orientation of the electric field with respect to the foliation of a rock sample (see Figure 3-1).

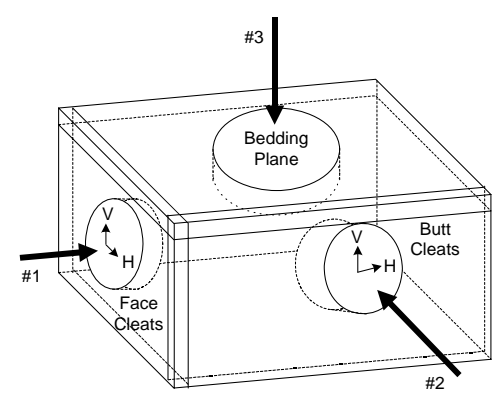

Figure 3-1. Three orientations of a rock sample.

Some characteristic values for the electrical properties of a variety of natural media are shown in Figure 3-2. The electrical conductivity of most natural media increases with frequency. The left end of the bar symbol in Figure 3-2 corresponds to low frequency values.

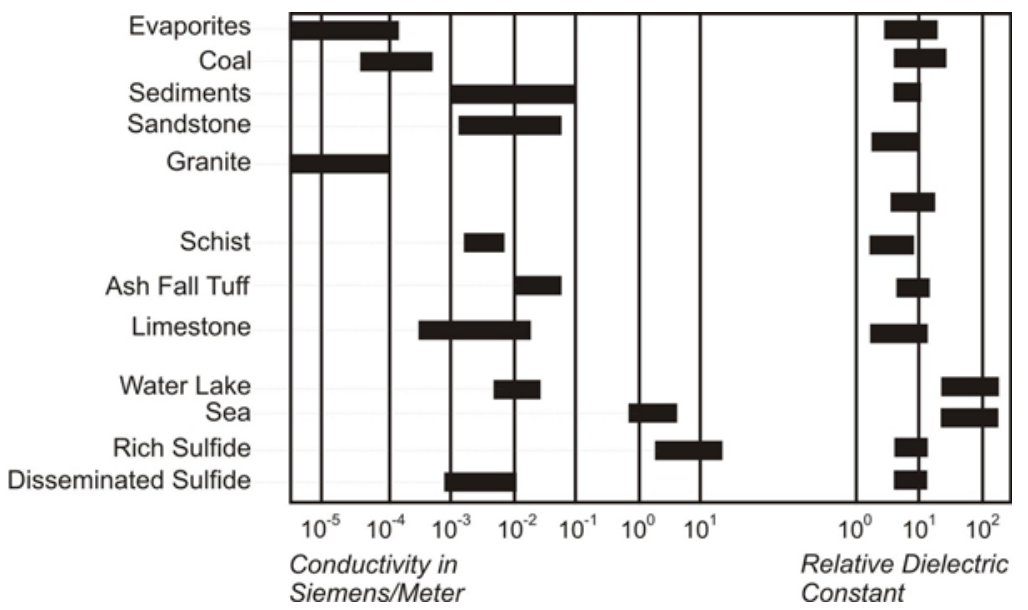

Figure 3-2. Range of electrical conductivity and relative dielectric constant for natural media. 
The electrical properties of dry coal and rock samples are shown in Figure 3-3 [6]. As shown in Figure 3-3, there is a decrease in dielectric constant (i.e., permittivity) and resistivity with increasing frequency, which is typical of most rocks. As a result of the decrease in resistivity, material losses are more pronounced at higher frequencies. The difference in resistivity or dielectric constant of shale and coal samples is most pronounced at lower frequencies. The data shown in Figure 3-3 correspond to measurements made on the face (i.e., primary) cleats of samples, in contrast to the bedding planes, which affect the performance of a radar in determining a boundary.

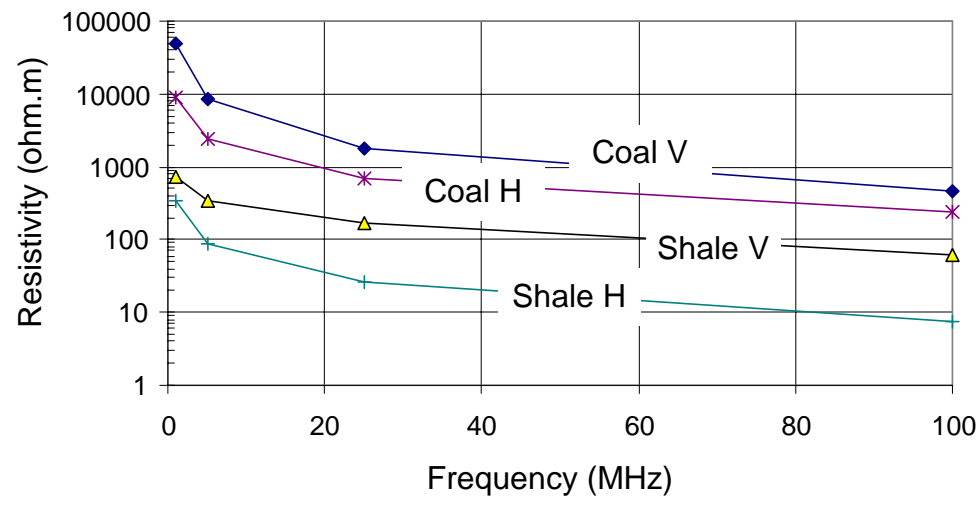

(a)

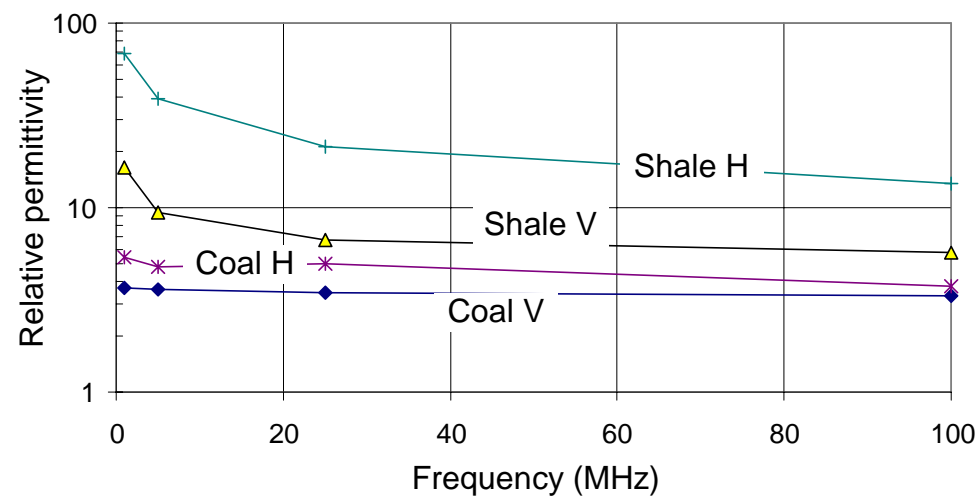

(b)

Figure 3-3. Electrical properties of dry coal and roof shale samples from an underground mine in Meigs County, Ohio. Vertical (V, E-field perpendicular to foliation) and horizontal (H, E-field parallel to foliation) polarizations are indicated: (a) resistivity; (b) relative dielectric constant. The data are reproduced from Ref. [6].

An HP-4192 network analyzer and an Agilent 16451B dielectric test fixture (see Figure 3-4) were used to determine the electrical properties of materials at low frequencies. This instrumentation is designed to measure the electrical properties of solid materials below $10 \mathrm{MHz}$ with a loss tangent below 10, which means that highly conductive materials cannot be tested with this setup. The material under test must be prepared as a disk-shaped sample, with a diameter between 1.6 and 2.2 inches and a thickness less than 0.4 inch. Also, the sample must have a smooth surface. The preparation of samples to meet such requirements can be a difficult task, especially for samples from fragile rocks. Due to sample imperfections, errors in measurements 
occur. Even without imperfections, test results vary between the samples of the same type of rock due to the heterogeneity of subsurface formations.

To make a measurement, the sample disk is placed between the two fixed, 2.5-inch parallel-plate electrodes of the dielectric probe kit (see Figure 3-4). The impedance analyzer measurers the complex impedance of the material sandwiched between the electrodes. This complex impedance is then related to the electrical properties of the material.

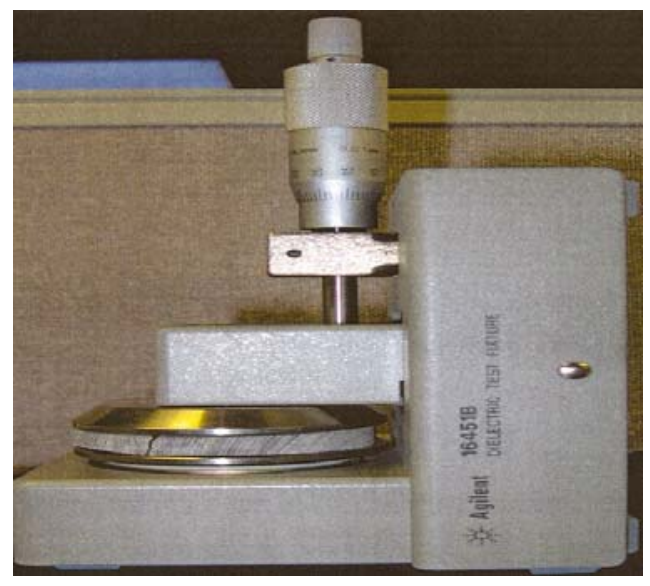

Figure 3-4. Agilent 16451B dielectric test fixture used for low-frequency testing of solid media.

The material tests for high frequencies (i.e., above $200 \mathrm{MHz}$ ) were performed with an Agilent $85070 \mathrm{E}$ dielectric probe kit connected to an Agilent 8753ES vector network analyzer. In this case, the sample must have one flat surface to lay flat against the calibrated, open-circuit coaxial probe. The instrumentation and the sample holder are shown in Figure 3-5. The diameter of the sample must be at least 0.8 inch. In contrast to the low-frequency instrumentation illustrated in Figure 3-4, the high-frequency instrumentation can measure highly conductive rocks.

The electrical properties of dried silty sandstone and basalt were measured from $20 \mathrm{kHz}$ to 3 GHz and are shown in Figures 3-6 and 3-7, respectively. A gap in the measurements occurs between $10 \mathrm{MHz}$ and $200 \mathrm{MHz}$. These properties indicate that basalt has a lower dielectric constant and loss than dried silty sandstone. Increasing the operating frequency decreases resistivity and dielectric constant values.

The rock samples that are extracted for laboratory measurements rarely retain their underground moisture content. Typically, these samples are sent for a chemical analysis where they are run through a centrifuge. In soil science and geophysical applications, the complex refractive index method (CRIM) typically is used to model the electrical properties of a composite material. The CRIM is based on the electrical properties of every constituting component and its volume fraction. Feng and Sen [7] modified the CRIM by incorporating the state of the moisture in rock (e.g., oil in water or water in oil) and used porosity and water saturation in determining the electrical properties of partially saturated rocks. Adding seawater to an oil-wet rock can dramatically affect its electrical properties by transforming the rock from a dielectric to a conductor. 

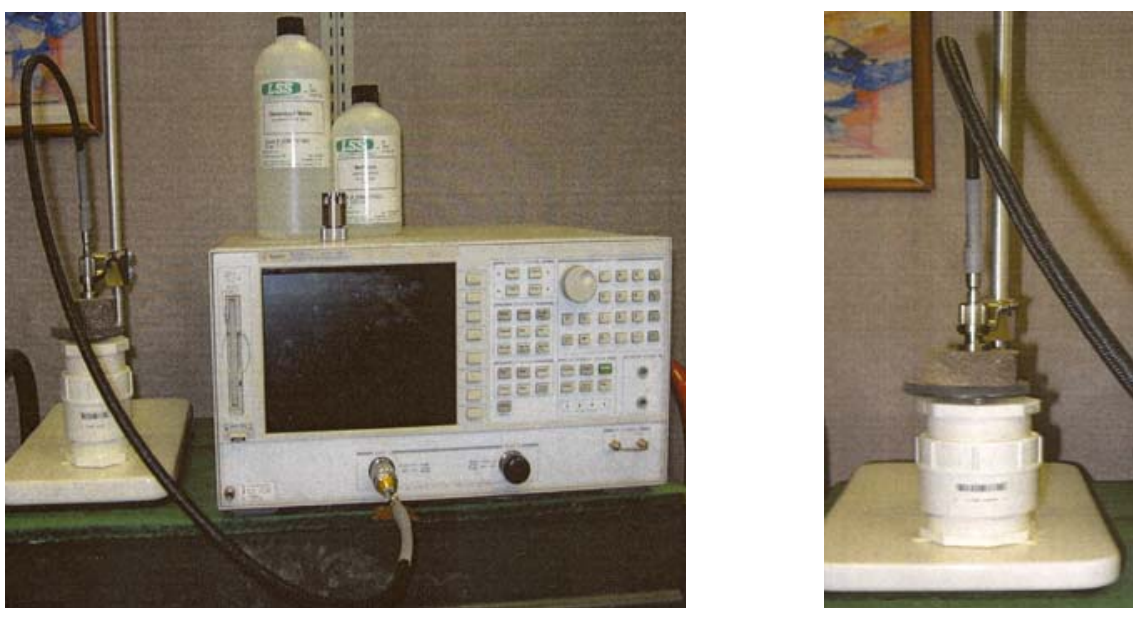

Figure 3-5. High-frequency instrumentation for measuring electrical properties of rocks.
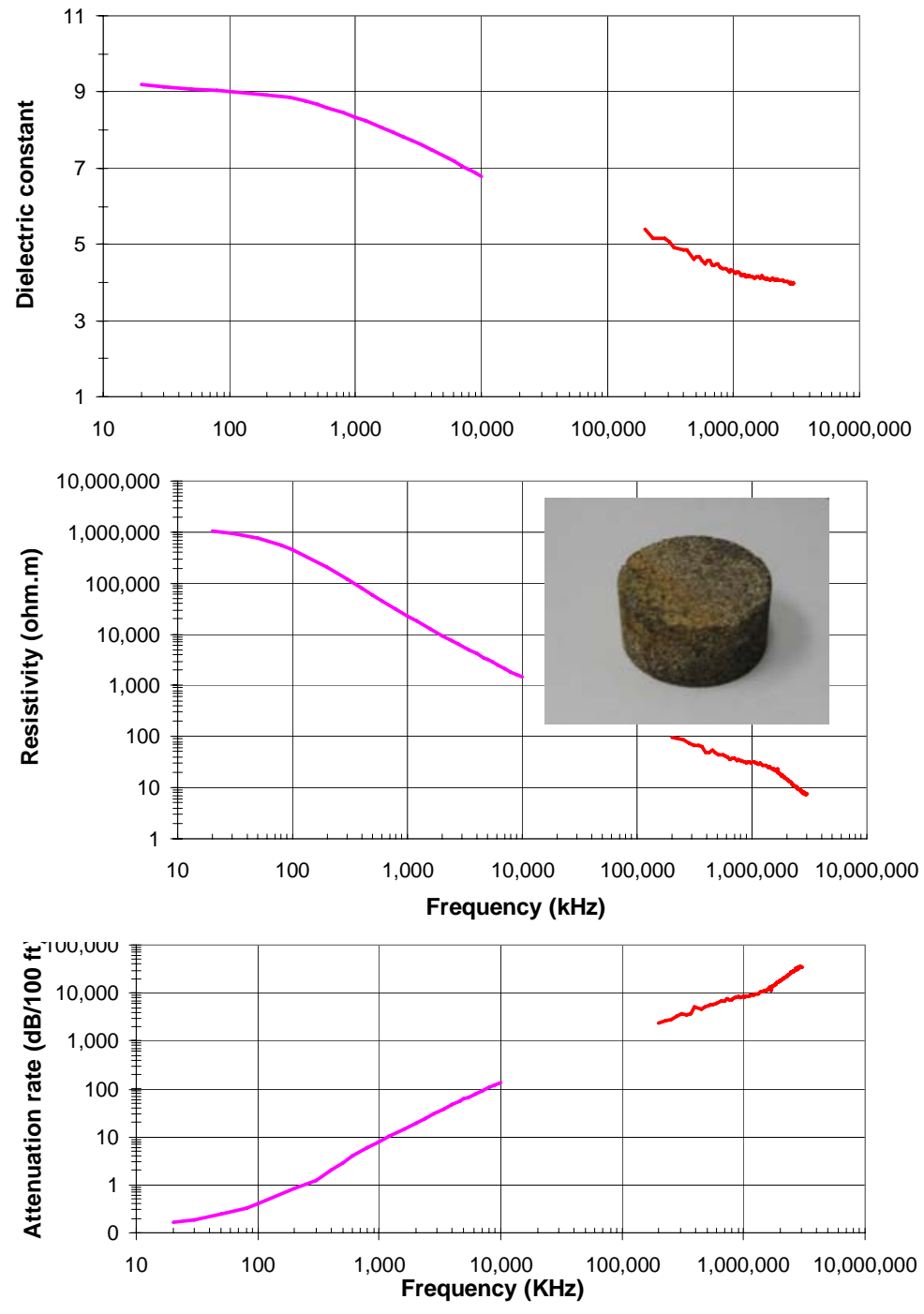

Figure 3-6. Measured electrical properties of a basalt sample. 

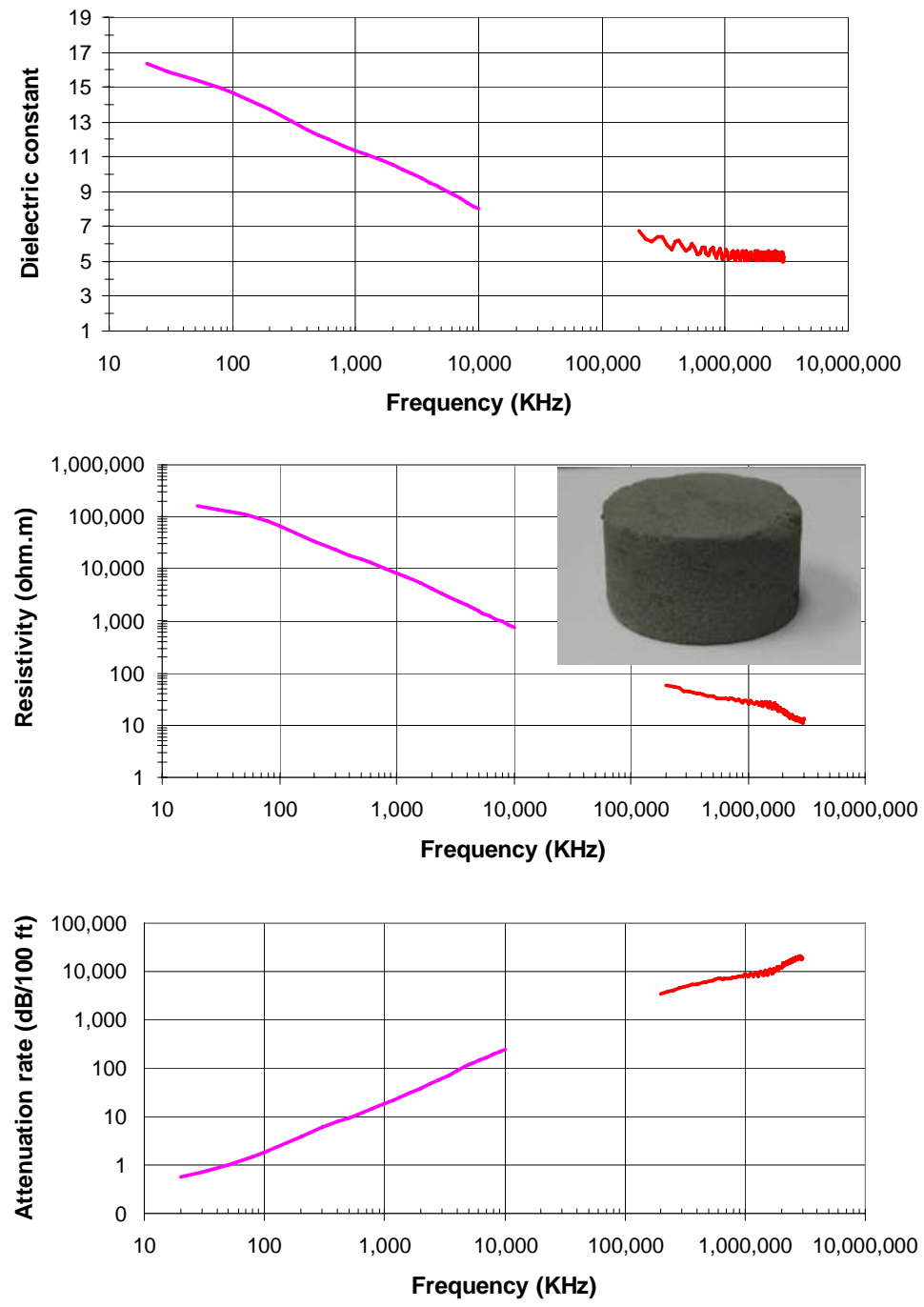

Figure 3-7. Measured electrical properties of a silty sandstone sample.

Figure 3-8 shows the results for bedding-plane measurements of the dielectric constant and resistivity for samples taken from the McElroy Coal Mine in the Pittsburgh seam. As indicated in Figure 3-8, contrast between coal and boundary rock is best observed at low frequencies. The measured resistivity values of coal and roof rock are approximately $400,000 \mathrm{ohm}-\mathrm{m}$ at $20 \mathrm{kHz}$. In comparison, measured resistivity values of oil-saturated limestone in the Jean-Marie formation in Canada and the boundary rock consisting of water-saturated limestone are about 150 and 5 ohm-m, respectively. Thus, at $20 \mathrm{kHz}$, the oil-saturated limestone would be classified as a conductor, whereas the Pittsburgh seam coal sample would be classified as a dielectric.

The dielectric constant in natural media is complex and can be given by

$$
\varepsilon^{*}=\varepsilon^{\prime}-\mathrm{i} \varepsilon^{\prime \prime}
$$

where $\varepsilon^{\prime}=$ the real part, and

$$
\varepsilon^{\prime \prime}=\text { the imaginary part. }
$$




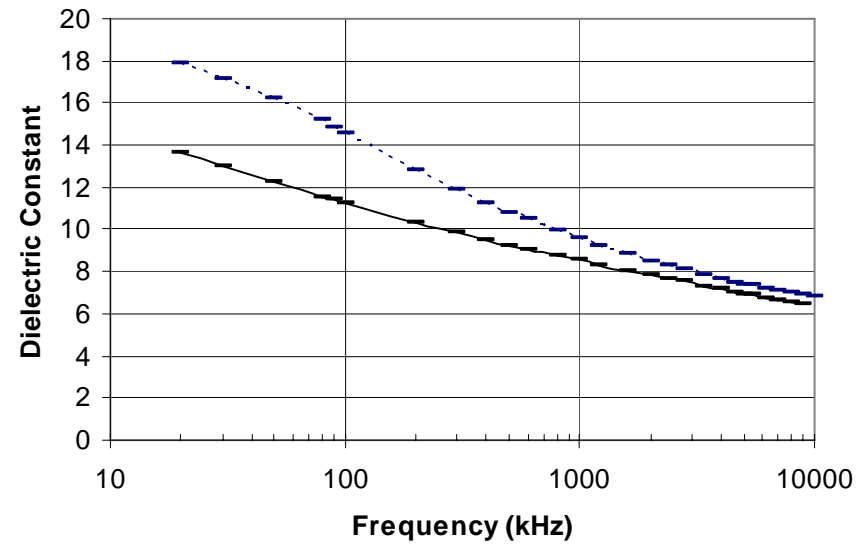

(a)

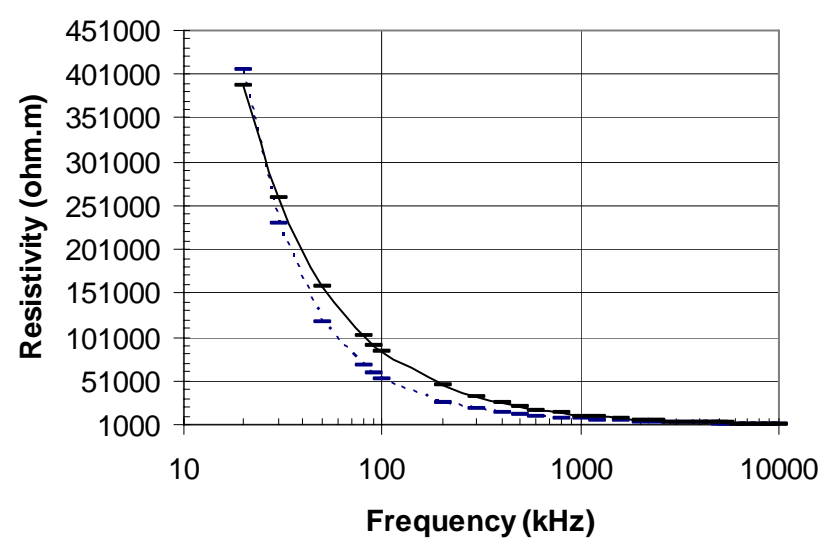

(b)

Figure 3-8. Comparison of the EM material properties from the bedding plane samples of coal (solid black) and roof rock (dashed blue): (a) dielectric constant; (b) resistivity.

The electrical conductivity is given by

$$
\sigma=\varepsilon^{\prime \prime} \omega
$$

The attenuation rate is expressed as

$$
\alpha=\omega \sqrt{\frac{\varepsilon^{\prime \prime} \mu}{2}} \text { in nepers per meter. }
$$

The electrical conductivity $(\sigma)$ of sedimentary rocks has been measured in Stolar's laboratory and shows a first-order dependence on frequency as shown in Figure 3-9.

Because of the complex nature of the natural media dielectric constant, the electrical conductivity is frequency dependent. The loss tangent, $\sigma / \omega \varepsilon$, is the ratio of the conduction to displacement current. 


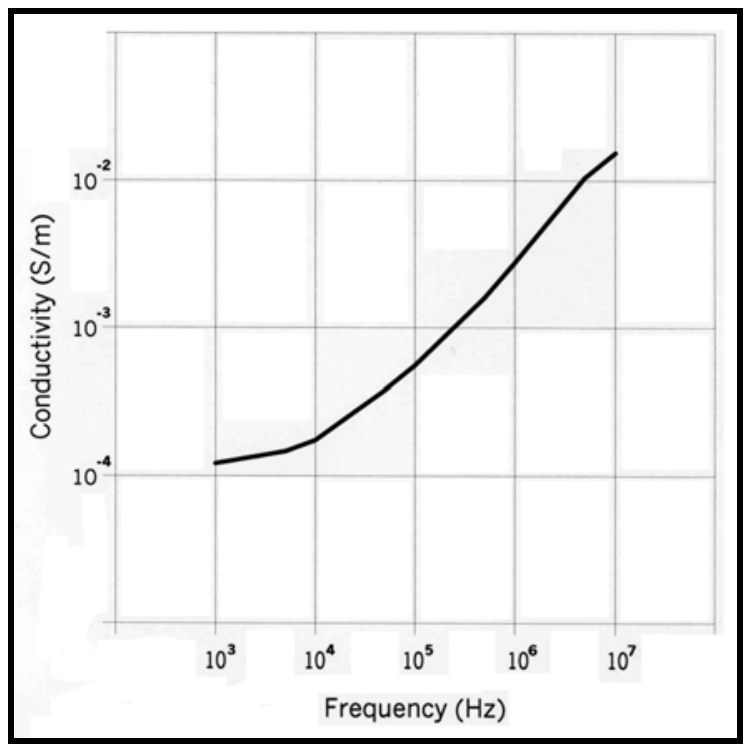

Figure 3-9. Electrical conductivity (Siemens $/ m$ ) of sedimentary rock versus frequency.

The attenuation rate $(\alpha)$ and phase shift $(\beta)$ values are shown in graphical form in Figure 3-10. The skin depth and wavelength of subsurface EM waves are shown in Figure 3-11.

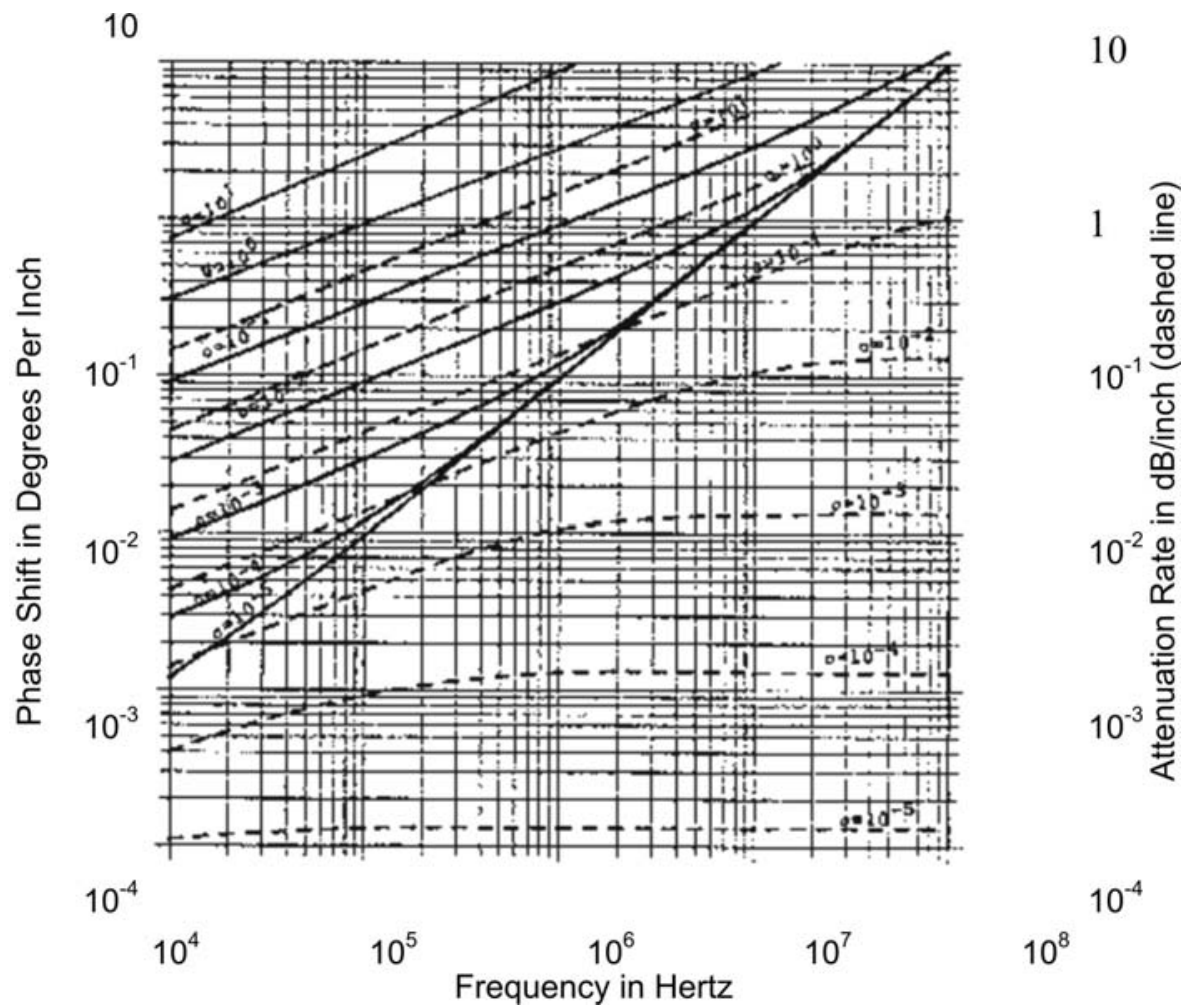

Figure 3-10. Attenuation rate and phase constant for a uniform plane wave propagating in a natural medium with a relative dielectric constant of 10. The bottom-to-top curves represent increases in natural media conductivity from $10^{-5}$ to $10^{1} \mathrm{~S} / \mathrm{m}$. 


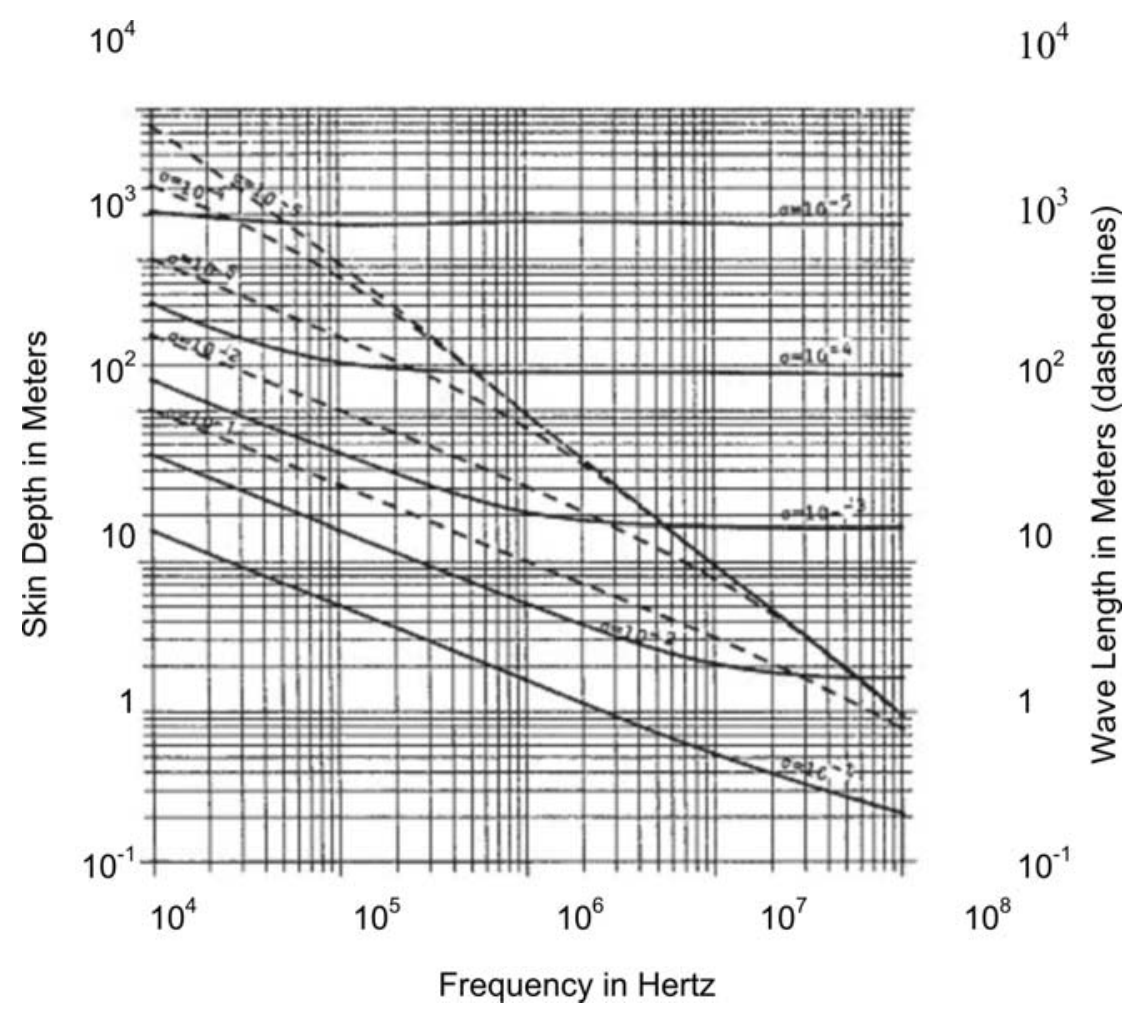

Figure 3-11. Skin depth and wavelength in a natural medium with a relative dielectric constant of 10. 


\subsection{Radar-Based Navigation Systems}

Horizontal directional drilling in an oil-saturated sandstone reservoir is illustrated in Figure 4-1. Instead of the trial-and-error drilling procedure requiring sidetracks in an undulating deposit, the borehole can be drilled following a geologic boundary in the reservoir by the use of radar-based measurement-while-drilling (MWD) navigation systems. With an MWD instrument (e.g., radar distance measurement) and automated steering, drilling costs can be decreased and recovery rates can be increased.

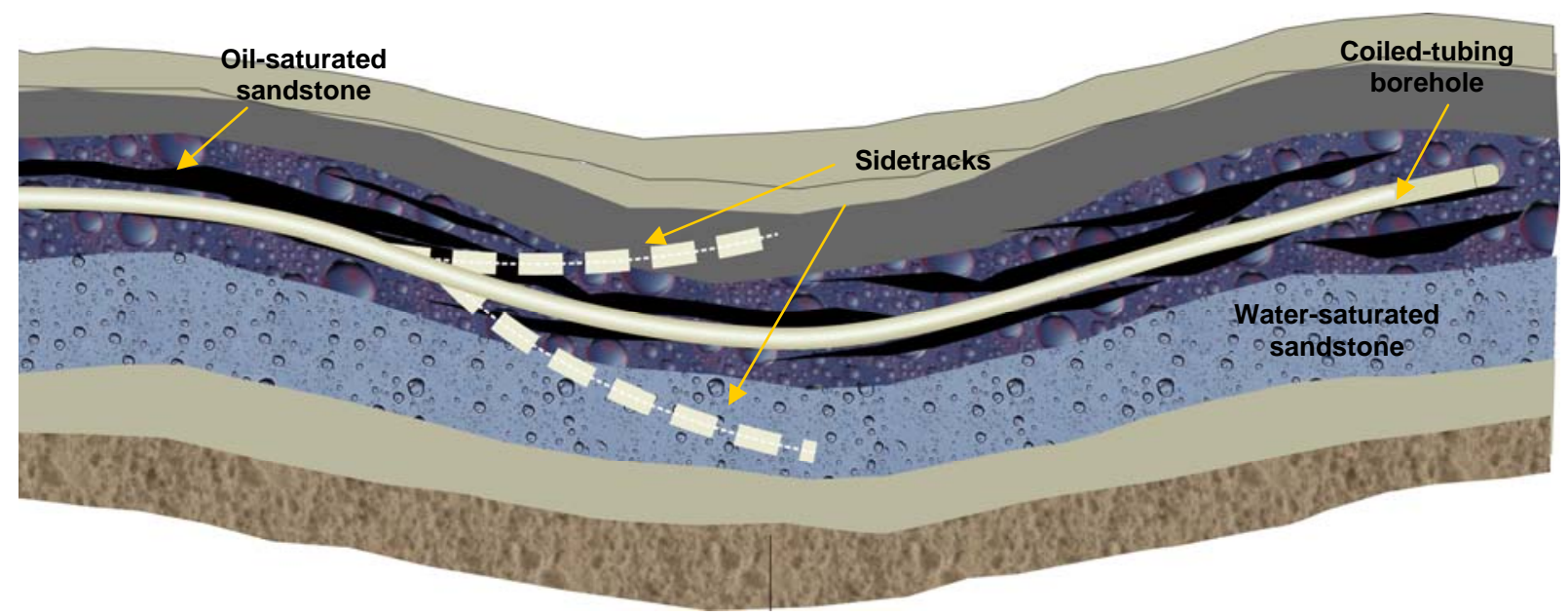

Figure 4-1. Vertical cross-section illustrating directional drilling in an oil-saturated sandstone reservoir.

The primary function of the Stolar Drill String Radar (DSR) equipment is to transmit electromagnetic (EM) waves into the boundary rock surrounding a wellbore, which will react to the subsurface geology and structure. The DSR is a very specialized radar, which can be installed in a bottom hole assembly typically deployed in coiled tubing or segmented drill pipe, capable of providing real-time guidance and navigation of the drilling process through unknown strata in hydrocarbon reservoirs.

\subsection{Theoretical Considerations}

\subsubsection{Basic Radar Background}

Radar (Radio-wave Detection and Ranging) instrumentation was developed during World War II to detect and track aircraft. Radar implies that transmitting and receiving antennas are collocated and operate as reflected (i.e., scattered) wave measuring instruments. These radar instruments solved the secondary field measurment (i.e., detection) problem by employing transmitted shortduration primary electromagnetic (EM) waves that travel in free space at the constant speed of light $(c)$ and illuminate the target at a distance $(d)$. The target reflects the secondary EM fields back to the radar after the round-trip travel delay time $\left(\mathrm{t}_{\mathrm{o}}\right)$.

The time dependence of the primary and reflected secondary radar waveform is illustrated in Figure 4-2. 


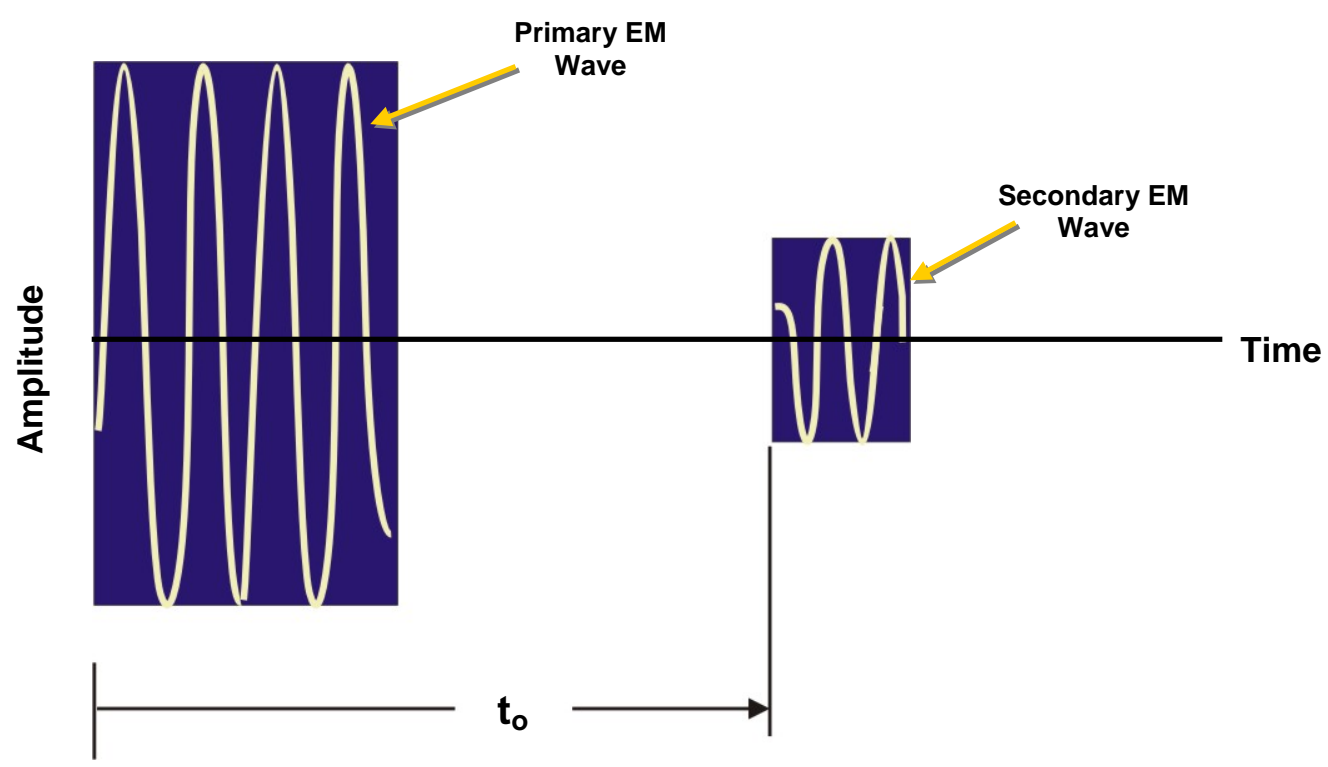

Figure 4-2. Short-duration pulse radar waveform.

The radar instrumentation measures the round-trip travel time $\left(\mathrm{t}_{\mathrm{o}}\right)$ and a rotating antenna determines the azimuth angle to the target. The travel distance to the target being tracked is given by

$$
d_{o}=\frac{c}{2 \mathrm{t}_{\mathrm{o}}} \text { in meters. }
$$

The design of radar instrumentation encountered the practical problem of high-energy primary wave leakage into the nearby receiving antenna. The high-energy leakage caused the receiving antenna and the associated electronic circuits to exhibit an impulse response. These circuits ring down as the transmitter leakage energy dissipates in the circuit. Because the sensitive radar receiver can be damaged during the ring-down time period, the receiver is switched off for a short duration of time during and following each transmission burst. This practice causes radar to be blind for short distances from the radar antenna location (i.e., within the near field given by $\lambda / 2 \pi)$. The far-field distance limitation occurs when the radar return (i.e., secondary EM wave) amplitude falls below the receiver inherent electrical noise. The receiver electrical noise $\left(\mathrm{e}_{\mathrm{N}}\right)$ is given by

$$
\mathrm{e}_{\mathrm{N}}^{2}=4 \mathrm{KTR} \mathrm{BW}
$$

where $\mathrm{T}=$ the temperature in degrees Kelvin,

$\mathrm{K}=$ the Boltzman's constant,

$\mathrm{R}=$ the equivalent resistance of the receiver circuit, and

$\mathrm{BW}=$ the noise bandwidth in Hertz of the receiver design.

The short-duration pulse radar transmitted EM wave exhibits a significant occupied bandwidth (BW). The transmitted waveform is illustrated in Figure 4-3. 

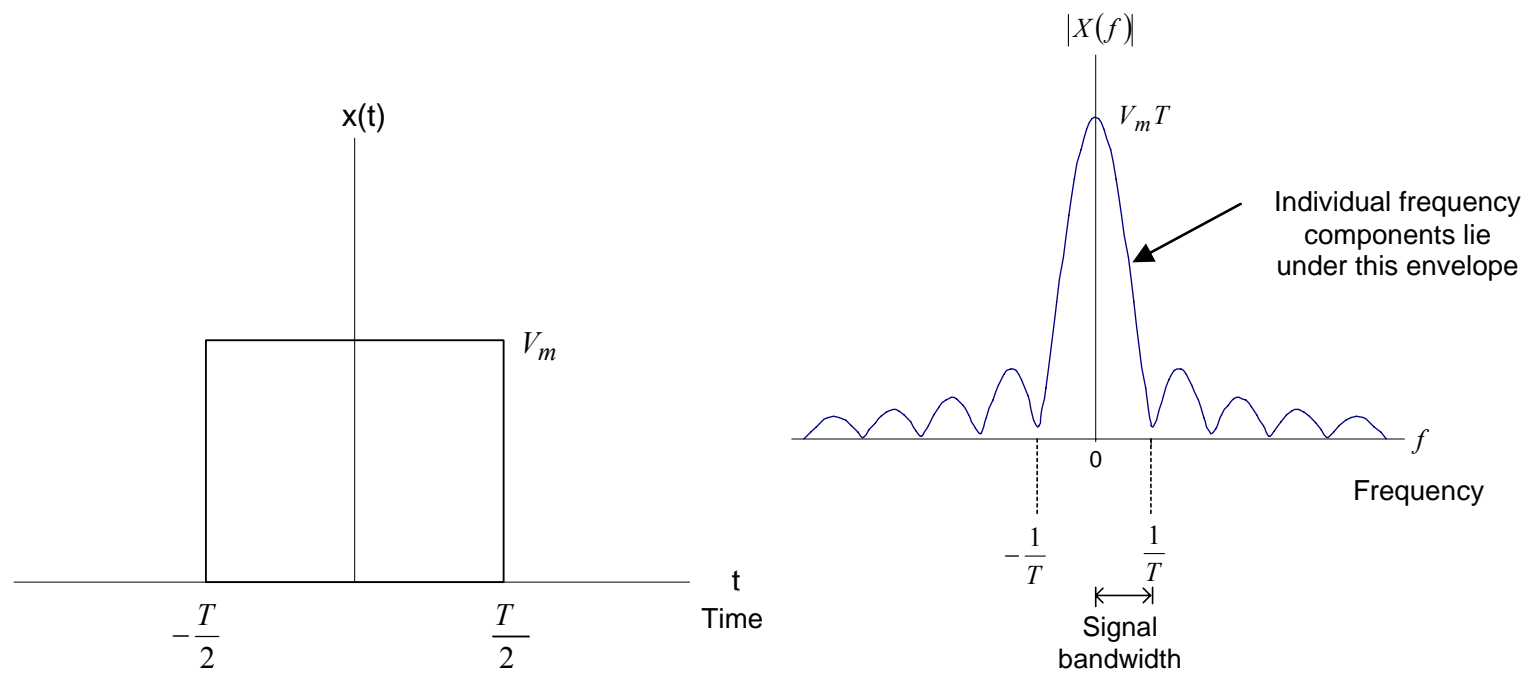

Figure 4-3. Time and frequency domain of a short-duration pulse radar.

The Fourier transform of the primary time-domain radar pulse is a frequency domain signal with multiple frequency components. Short-duration pulse radars commonly have individual frequency components (i.e., sinusoidal signals) that occupy a bandwidth greater than $25 \mathrm{MHz}$. The large occupied bandwidth of the short-duration pulse requires that the passband of the radar antenna be at least as large as the occupied bandwidth. The antenna must be matched to the characteristic impedance $\left(\mathrm{Z}_{\mathrm{O}}\right)$ of the directional coupler. The antenna design reflection coefficient given by

$$
\Gamma=\frac{Z_{L}-Z_{O}}{Z_{L}+Z_{O}}
$$

must be as near to zero as possible to avoid the mismatch loss of $20 \log _{10} \Gamma$.

The large occupied bandwidth deteriorates maximum receiver sensitivity by the degradation factor

$$
\Delta_{\max }=20 \log _{10}(\mathrm{BW})^{1 / 2} \text { in decibels } .
$$

The penalty paid in loss of sensitivity in a short-pulse duration radar is approximately $74 \mathrm{~dB}$ when compared to a $1-\mathrm{Hz} \mathrm{BW}$ stepped-frequency radar receiver.

From communications theory, the optimum receiver design to maximize the receiver sensitivity to a sinusoidal signal embedded in electrical noise is a synchronized (i.e., autocorrelation) detector [8]. The synchronized receiver design drives the noise bandwidth to less than $1 \mathrm{~Hz}$. Arguably, digital sampling of the radar secondary reflected signal and digital signal processing by averaging can achieve the same receiver threshold sensitivity. However, this argument fails to take into consideration the feed of the digital sample through signal limitation. 


\subsubsection{Radar in Natural Media}

Electromagnetic wave reflections at air-oil-saturated limestone and limestone-boundary rock interfaces when the receiving antenna is collocated with the transmitting antenna are illustrated in Figure 4-4. The radar generates an EM wave that travels through the oil-saturated limestone to the boundary layer. The EM wave is partially reflected at the antenna-oil-saturated limestone interface and again at the limestone-boundary rock interface. The distance $(\mathrm{R})$ to the boundary layer is determined by the well-known radar equation

$$
R=\frac{1}{2} v \tau \text { meters, }
$$

where $v$ is the velocity of the EM wave in the oil-saturated limestone in meters per second, and $\tau$ is the round trip travel time in seconds.

The travel time $(\tau)$ is determined by processing the reflected signal in the radar electronics. The dispersive nature of natural media causes the occupied bandwidth frequency components to travel at different speeds, which distorts the returning secondary EM waves.

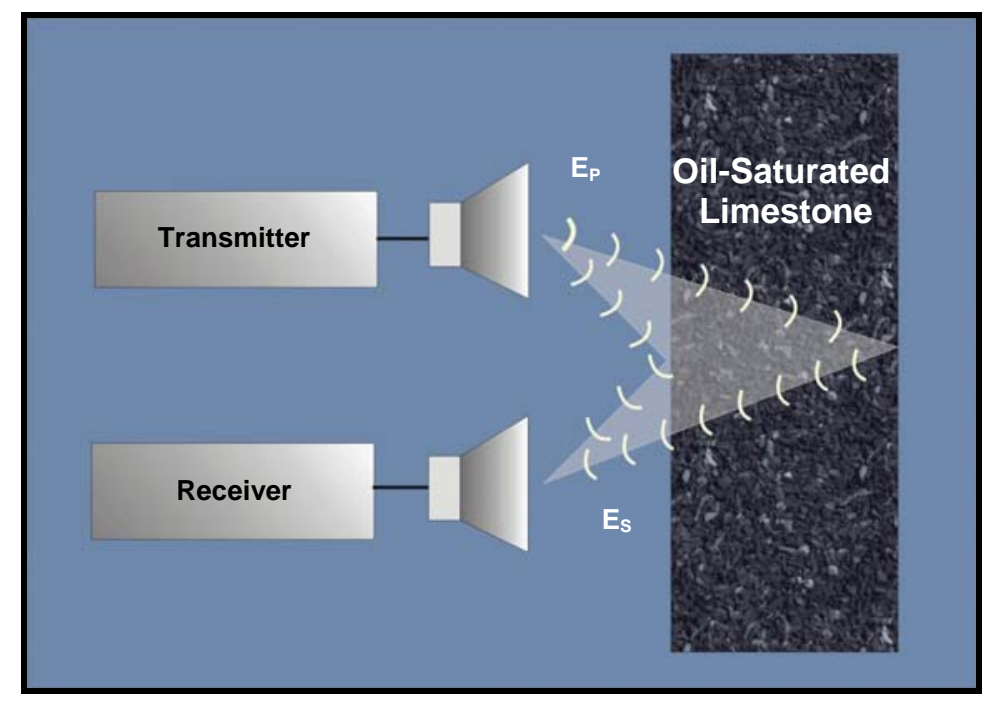

Figure 4-4. Radar reflections at boundaries.

The primary EM wave electric field component $\left(\mathrm{E}_{\mathrm{p}}\right)$ travels to and is reflected by the first air-oilsaturated limestone interface, creating a secondary electric field component $\left(\mathrm{E}_{\mathrm{s}}\right)$ that arrives back at the receiving antenna. Part of the primary wave travels through the first interface and is reflected by the oil-saturated limestone-boundary rock interface located at distance (R). The reflected wave arrives at the receiving antenna with a delay of $\tau$ seconds after leaving the transmit (TX) antenna and being reflected from the second interface.

The reflected electric field component $\mathrm{E}_{\mathrm{S}}$ from the nearby oil-saturated interface can be determined from the reflection coefficient $(\Gamma)$ expressed mathematically as 


$$
\Gamma=\frac{E_{R}}{E_{i}}=\frac{Z_{2}-Z_{1}}{Z_{2}+Z_{1}},
$$

where the impedance of the natural medium is given by

$$
\begin{aligned}
& Z=\frac{\sqrt{\frac{\mu}{\varepsilon}}}{\sqrt{1-i \frac{\sigma}{\omega \varepsilon}}}, \\
& \left|Z_{i}\right|=\frac{\sqrt{\frac{\mu}{\varepsilon}}}{\left[1+\left(\frac{\sigma}{\omega \varepsilon}\right)^{2}\right]^{\frac{1}{4}}} \\
& |Z|=\left\{\begin{array}{l}
\left|\sqrt{\frac{i \omega \mu}{\sigma}}\right|=\sqrt{\frac{\omega \mu}{\sigma}} ; \frac{\sigma}{\omega \varepsilon}>>1 \\
\frac{377}{\sqrt{\varepsilon_{r}}} ; \frac{\sigma}{\omega \varepsilon}<<1
\end{array}\right.
\end{aligned}
$$

The transmission through the interface is given by

$$
T=\frac{2 Z}{Z+Z_{o}} \text {. }
$$

Because the reflected wave from the oil-saturated limestone interface predominates the reflected wave from the boundary rock radar instruments must be designed to measure a much smaller secondary field in the presence of a larger primary field.

The EM wave reflection process is more complicated because of internal reflections occurring within the oil-saturated limestone and EM wave energy absorption (i.e., heat loss). This phenomenon is illustrated in Figure 4-5.

The EM wave energy $\left(E_{S 1}\right)$ reflected from the first interface is several orders of magnitude greater than the energy $\left(\mathrm{E}_{\mathrm{S} 2}\right)$ reflected from the second interface. The first interface reflection presents a number of technical challenges in the radar detection problem. When the first interface reflected wave energy reaches the antenna, the antenna response exhibits a radar "ring" time period before reaching steady state. The ring time period will last for a few nanoseconds and interfere with detection near the first interface and weak reflections from the second interface. Although the ring time period is easy to visualize in a pulse radar, a similar phenomenon occurs 
in a frequency-modulated, continuous wave (FMCW) or stepped-frequency continuous wave (SFCW) radar because of the finite frequency range in the frequency domain measurement. The transformation of frequency-domain-measured data to the time domain forms a ring time domain response.

Figure 4-5. Electromagnetic wave energy flow in a drill string radar application.

The reflection from the first interface can saturate (i.e., overload) sensitive amplifiers that are designed to amplify the much weaker reflected wave from the boundary layer interface. In fact, antennas designed for near-field impedance measurement are needed to measure distance in thin oil-saturated limestone layers.

Because of space limitations, directional couplers have been developed to allow a single microwave antenna to transmit and receive radar EM waves. Figure 4-6 is a block diagram of a radar system designed with a directional coupler.

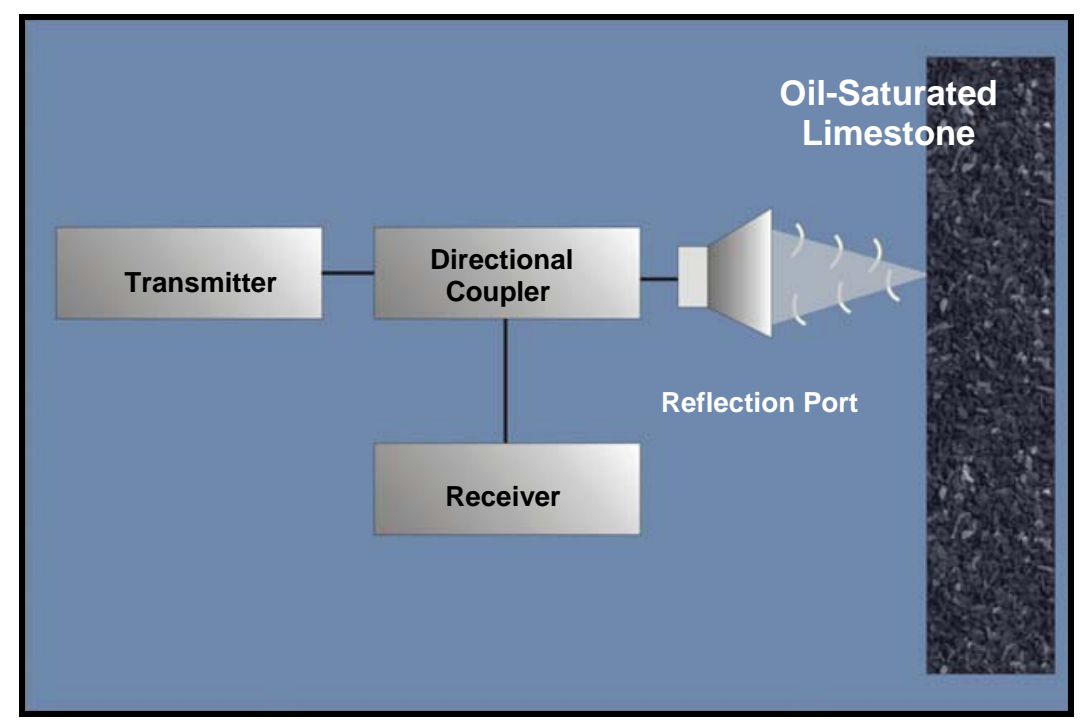

Figure 4-6. Radar with a directional coupler. 
The secondary reflected signal traveling toward the radar appears at the reflection port of the directional coupler. The receiver measures the reflected signal from the first air-oil-saturated limestone and second limestone-boundary rock interfaces. Directional couplers have a directivity limitation that is the leakage of the transmitter signal into the reflection port. Welldesigned directional couplers exhibit a directivity of $100 \mathrm{~dB}$, which is achieved with calibration algorithms.

Impulse and frequency-domain are the two broad categories of all of the reflection-type radar systems. Measurements in one category can be transformed to the other by the aid of Fourier transformation of the received signal. Stolar employs frequency-domain type radar systems. The two major types of frequency-domain radars are stepped-frequency, continuous-wave (SFCW) and frequency-modulated, continuous-wave (FMCW).

The transmission and reception of radio waves with these two radar systems are graphically depicted in Figure 4-7. The received signal of an FMCW radar system is at a different frequency from the transmitted frequency when there is a single target present (see Figure 4-7). This beat frequency $\left(f_{b}\right)$ identifies the distance $(r)$ from the target. The FMCW radar systems are used for exploration of deep targets. By filtering out the unwanted beat frequencies generated by close scatterers, an FMCW radar system can decipher the distance to a faraway target. On the other hand, a radar system is termed stepped-frequency when transmission and reception of radio waves are made at the same frequency by means of discrete, equally spaced frequencies. An inverse Fourier transform converts the frequency-domain data to a temporal format in either FMCW or SFCW radar systems. Once the speed of wave inside the medium is known (i.e., once the dielectric constant of the medium is known), the time-domain data can be correlated to spatial coordinates.

Figure 4-7. Functional diagram of the transmitted and received signals in (a) FMCW and (b) SFCW radar systems. Transmitted and received signals are shown in blue and red, respectively.

When the two orthogonal (in-phase and quadrature) components of the received signal are measured, then the bandwidth $(B W)$ of the measurement and spacing between frequencies $(\Delta f)$, 
respectively, determine the spatial resolution of the measurements $(\Delta R)$ and the maximum range of operation $\left(R_{\max }\right)$ by the following formulas:

$$
\begin{gathered}
\text { Resolution }=\Delta R=\frac{c}{2 B W \sqrt{\left|\varepsilon / \varepsilon_{0}\right|}} \\
\text { Maximum Range }=R_{\max }=\frac{c}{2 \Delta f \sqrt{\left|\varepsilon / \varepsilon_{0}\right|}},
\end{gathered}
$$

where $c=$ the speed of light in vacuum $\left(3 \times 10^{8} \mathrm{~m} / \mathrm{s}\right)$,

$\varepsilon_{o}=$ the dielectric constant of free space, and

$\varepsilon=$ the complex dielectric constant of the medium in which the radio waves propagate.

In the case of low-loss earthen materials, the imaginary component of the complex dielectric constant is usually much less than the real component of the complex dielectric constant and can be ignored, viz.

$$
\varepsilon=\varepsilon^{\prime}-j \varepsilon^{\prime \prime} \cong \varepsilon^{\prime}=\varepsilon_{0} \varepsilon_{r}
$$

Therefore, the modulus term in Equations (4-11) and (4-12) can be replaced by the relative dielectric constant, $\varepsilon_{r}$, for a low-loss material. To afford a deeper penetration of the radar signals inside the Earth, providing the required bandwidths at the lowest possible operating frequencies is desirable.

\subsubsection{Stepped-Frequency, Continuous-Wave Radar}

To achieve increased detection range, a stepped-frequency, continuous-wave (SFCW) radar has been designed to generate the frequency-domain frequency components shown in Figure 4-3. This class of radar maximizes the detection range because the signal detection process can be optimized by autocorrelation. The detection process is optimal in the sense that the smallest possible sinusoidal signal can be detected in electrical noise [9]. Vary small detection noise bandwidth $(\mathrm{BW})$ is especially important in maximizing detection range. For each frequency component, the receiver mixdown frequency transposition process can be synchronized with the crystal frequency generator in the transmitter. Vary small receiver bandwidths can be achieved with SFCW radar. Since the total phase shift in each frequency component is measured, dispersion in the natural media can be determined. The radar requires sufficient time to transmit and measure the frequency-domain, continuous-wave signals. The number of individual frequencies required in the synthetic radar pulse depends upon the resolution required for the specific application for which the radar is being utlized.

The range and resolution of a number of stepped-frequency radar systems are given in Table 4-1, assuming that $\varepsilon_{r}=4$ for the low-loss material surrounding the radio waves. Reasonable bandwidths are needed to resolve the reflection from large objects. A very large bandwidth is needed to resolve two close reflecting surfaces. 
Table 4-1. Maximum range $\left(R_{\max }\right)$ and resolution $(\Delta R)$ of a stepped-frequency radar system with operating bandwidth $(B)$ and stepping frequency $(\Delta f)$.

\begin{tabular}{|c|c|c|c|c|c|}
\hline $\begin{array}{c}\text { Bandwidth } \\
(\mathrm{MHz})\end{array}$ & $\begin{array}{c}\text { Resolution } \\
(\text { feet })\end{array}$ & $\begin{array}{c}\text { Stepping } \\
\text { Frequency } \\
(\mathrm{MHz})\end{array}$ & $\begin{array}{c}\text { Maximum } \\
\text { Range } \\
(\mathrm{feet})\end{array}$ & $\begin{array}{c}\text { Stepping } \\
\text { Frequency } \\
(\mathrm{MHz})\end{array}$ & $\begin{array}{c}\text { Maximum } \\
\text { Range } \\
\text { (feet) }\end{array}$ \\
\hline 1000 & 0.25 & 100 & 2.5 & 50 & 5.0 \\
\hline 500 & 0.5 & 50 & 5.0 & 10 & 25.0 \\
\hline 100 & 2.5 & 10 & 25.0 & 5 & 50.0 \\
\hline 50 & 5.0 & 5 & 50.0 & 1 & 250.0 \\
\hline
\end{tabular}

The range and resolution capabilities of an underground radar system depend on the EM properties of the geological materials under investigation, namely, the dielectric constant and the resistivity. A radar system detects the EM contrast between various media. Since the EM properties of rocks are frequency-dependent, a judicious choice in determining the operating frequency is paramount to the performance of the radar system. For example, a radar system will not work if large losses (i.e., very low resistivity values) in the surrounding medium are encountered.

The wider the frequency band of a radar, the higher the resolution as is evident in Equation (411). The range resolution is given in Equation (4-12). Results from Equations (4-11) and (4-12) are shown in Figure 4-8 for the proper operation of a SFCW radar.

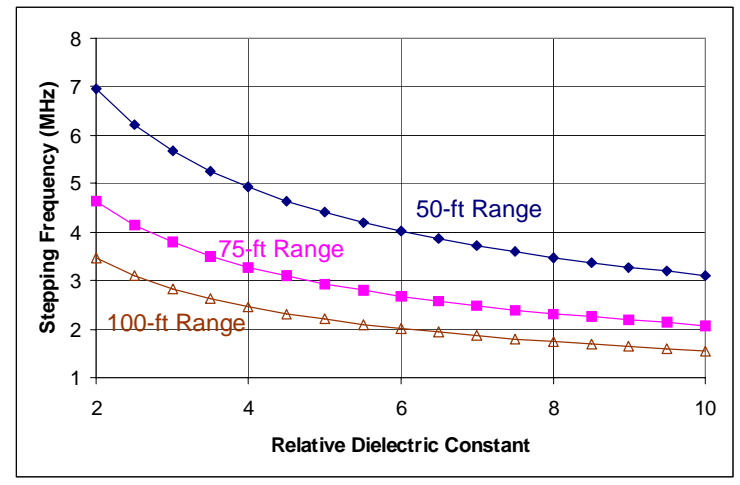

(a)

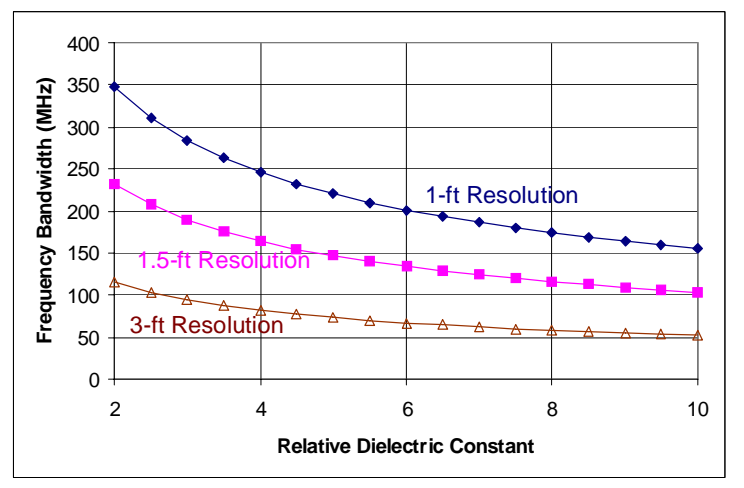

(b)

Figure 4-8. Stepping frequency (a) and bandwidth (b) required for the proper operation of an SFCW radar system.

\subsubsection{Frequency-Modulated, Continuous-Wave Radar}

Frequency-modulated, continuous-wave (FMCW) radars have been developed for use in aircraft altimeters. An advantage of this class of radar is that a narrow-band patch antenna can be used in place of a typical wideband microwave antenna using bow tie or horn designs.

Skolnik [10] describes the FMCW radar in great detail. The mathematical form of the receiver mixdown signal is represented by 


$$
A \sin \left[\omega_{0} \mathrm{t}+\phi_{0} \Delta \omega \cos \left(\omega_{\mathrm{m}} \mathrm{t}+\phi_{\mathrm{m}}\right)\right]
$$

where $\omega_{0}=2 \pi \mathrm{f}_{0}$ and $\mathrm{f}_{0}$ is the carrier frequency,

$\phi_{0}=$ the round trip phase shift (i.e., rotation) of the EM wave on the entire reflection path,

$\Delta \omega=$ the peak frequency deviation of the transmitter in Hertz,

$\omega_{\mathrm{m}}=2 \pi \mathrm{f}_{\mathrm{m}}$ and $\mathrm{f}_{\mathrm{m}}$ is the modulation frequency, and

$\phi_{\mathrm{m}}=$ the round trip phase shift (i.e., rotation) of modulation wave on the entire reflection path.

The mathematical form of the transmitted signal is represented by

$$
\sin \left(\omega_{0} t+\frac{\Delta \omega}{2 \omega_{m}} \sin \omega_{m} t\right)
$$

Sinusoidal waveforms with sinusoidal arguments can be mathematically expanded in Bessel functions of the first kind. The receiver mixdown (i.e., difference frequency) signal may be written as

$$
\begin{aligned}
\mathrm{V}_{\mathrm{D}}= & \mathrm{J}_{0}(\mathrm{D}) \cos \left(2 \pi \mathrm{f}_{\mathrm{d}} \mathrm{t}-\phi_{0}\right)+2 \mathrm{~J}_{1}(\mathrm{D}) \sin \left(2 \pi \mathrm{f}_{\mathrm{d}} \mathrm{t}-\phi_{0}\right) \cos \left(2 \pi \mathrm{f}_{\mathrm{m}} \mathrm{t}-\phi_{\mathrm{m}}\right) \\
& -\mathrm{J}_{2}(\mathrm{D}) \cos \left(2 \pi \mathrm{f}_{\mathrm{d}} \mathrm{t}-\phi_{0}\right) \cos 2\left(2 \pi \mathrm{f}_{\mathrm{m}} \mathrm{t}-\phi_{\mathrm{m}}\right) \\
& -\mathrm{J}_{3}(\mathrm{D}) \sin \left(2 \pi \mathrm{f}_{\mathrm{d}} \mathrm{t}-\phi_{0}\right) \cos 3\left(2 \pi \mathrm{f}_{\mathrm{m}} \mathrm{t}-\phi_{\mathrm{m}}\right) \\
& +\mathrm{J}_{4}(\mathrm{D}) \cos \left(2 \pi \mathrm{f}_{\mathrm{d}} \mathrm{t}-\phi_{0}\right) \cos 4\left(2 \pi \mathrm{f}_{\mathrm{m}} \mathrm{t}-\phi_{\mathrm{m}}\right)+2 \mathrm{~J}_{5}(\mathrm{D}) \ldots
\end{aligned}
$$

where $\mathrm{J}_{0}, \mathrm{~J}_{1}, \mathrm{~J}_{2}$, etc. $=$ Bessel functions of first kind of order $0,1,2$, etc., respectively,

$\mathrm{D}=\left(\Delta \mathrm{f} / \mathrm{f}_{\mathrm{m}}\right) \sin \left(2 \pi \mathrm{f}_{\mathrm{m}} \mathrm{R}_{0} / \mathrm{c}\right)$,

$\mathrm{R}_{0}=$ distance to target at time $\mathrm{t}=0$,

$\mathrm{c}=$ velocity of propagation,

$\mathrm{f}_{\mathrm{d}}=2 \mathrm{v}_{\mathrm{r}} \mathrm{f}_{0} / \mathrm{c}=$ Doppler frequency shift,

$\mathrm{v}_{\mathrm{r}}=$ relative velocity of target with respect to radar,

$\phi_{0}=$ phase shift approximately equal to angular distance $4 \pi \mathrm{f}_{0} \mathrm{R}_{0} / \mathrm{c}$, and

$\phi_{\mathrm{m}}=$ phase shift approximately equal to $2 \pi \mathrm{f}_{\mathrm{m}} \mathrm{R}_{0} / \mathrm{c}$.

The mixdown signal in the receiver is processed in a digital signal processor to bandpass filter and detect the modulation frequency $\left(\mathrm{f}_{\mathrm{m}}\right)$ and harmonics $\mathrm{H}=2,3,4, \mathrm{~m}$. The algorithm that processes the modulation signals determines the detection range.

\subsubsection{Near-Field Radar Considerations}

Conventional radar technology, including both SFCW and FMCW radar systems, is "blind" in the near field. To overcome this limitation, Stolar's research team and Dr. David G. Chang 
(President of Polytechnic Institute of New York) and Dr. Dickey Arndt of the NASA Johnson Space Flight Center determined that a resonant microstrip patch antenna (RMPA) could measure near-field conditions (see Figure 4-9).

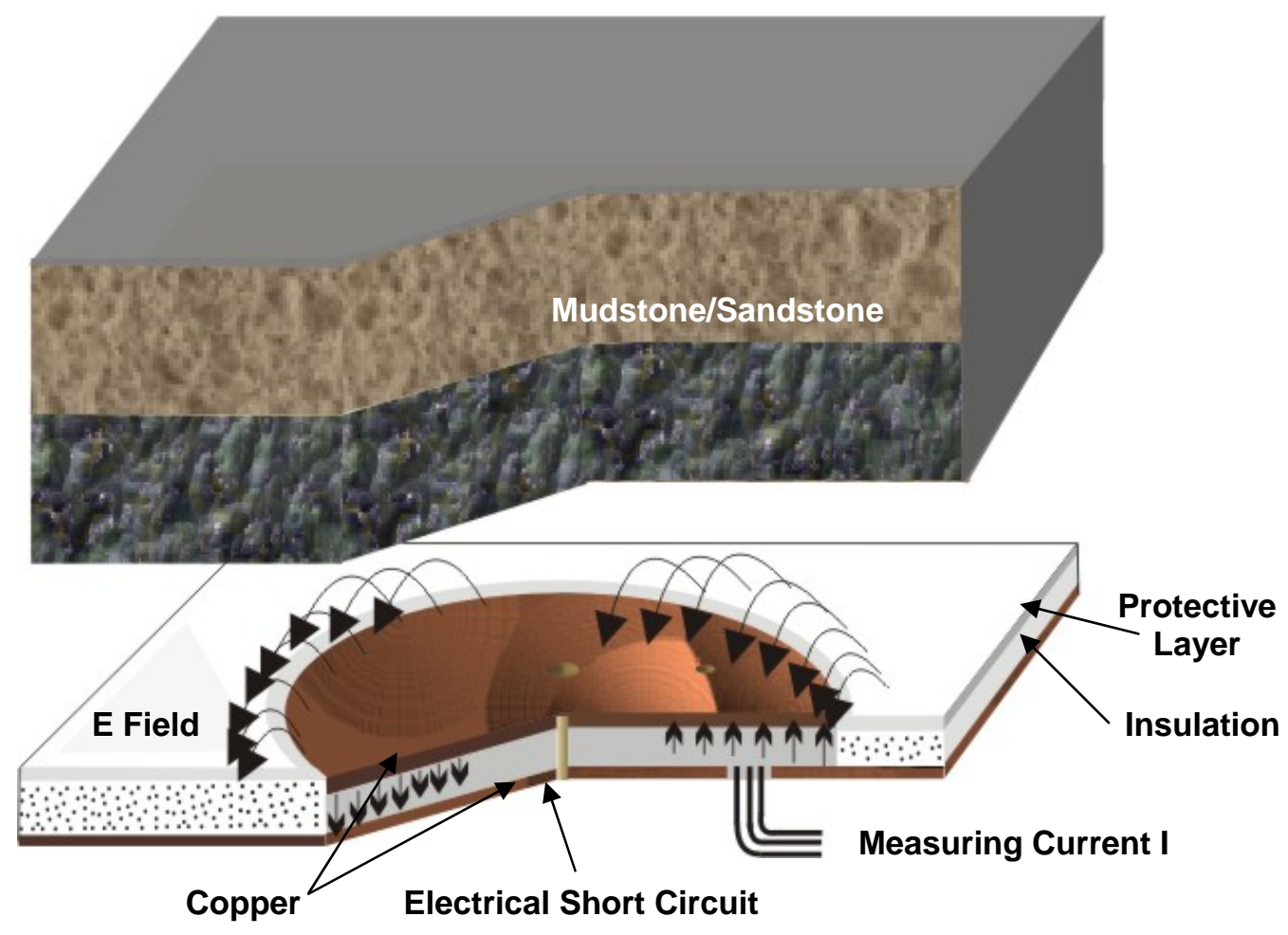

Figure 4-9. Resonant microstrip patch antenna (RMPA).

Dr. Robert Kelly of the Los Alamos National Laboratory determined that the sensor is a resonant cavity operating in the $\mathrm{TM}_{11}$ mode. The cavity resonator is constructed with a thin copper sheet on each side of an insulating layer. The measurement electronics generate a current (I) that flows in the coaxial cable to a short antenna in the insulator. The antenna produces a primary vertically polarized electric field that travels away from the antenna. The inward traveling field arrives at the center copper post and is reflected toward the edge of the copper patch. This secondary field arrives at the edge of the patch where it is added to the primary electric field. At the resonant frequency of the RMPA, the primary and secondary fields add together and achieve maximum value. At the edge of the patch, the summed fields fringe and exit the cavity as illustrated by the curved line fields in Figure 4-9. Along the opposite edge of RMPA, fringing fields also form. The fringing field creates a horizontally polarized field that travels upward through the oil-bearing zone and intersects the boundary rock. The reflected wave returns to the RMPA and re-enters the cavity. The reflected wave vectorally adds to the electric field at the coaxial cable antenna location. The ratio of the electric field (Z) to the current (I) is the antenna impedance, viz.

$$
Z=\frac{E}{I} .
$$

The impedance varies with the distance to the boundary rock as illustrated in Figure 4-10. 


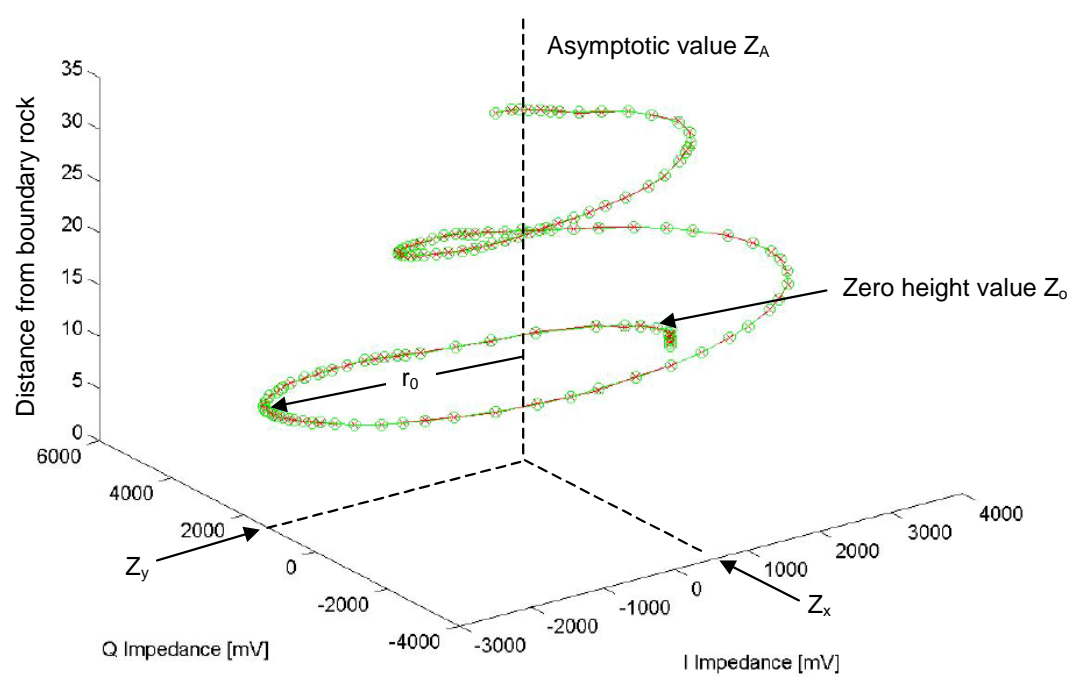

Figure 4-10. Impedance change with distance to the boundary rock. Each point on the impedance spiral corresponds to a distance in inches.

\subsubsection{Advanced Radar Design}

An advanced radar design is based on the transmission of a predistorted radar waveform combined with a software algorithm developed to suppress the reflection from the first interface (e.g., a wellbore). This process requires the precise control of frequency and phase in the pair of continuous waves in the transmit waveform. Control of the magnitude of the pair of continuous waves is also required in the predistorted waveform. The impact of the suppression of the first interface reflection is illustrated in Figure 4-11 for a constant modulation frequency SFCW radar.

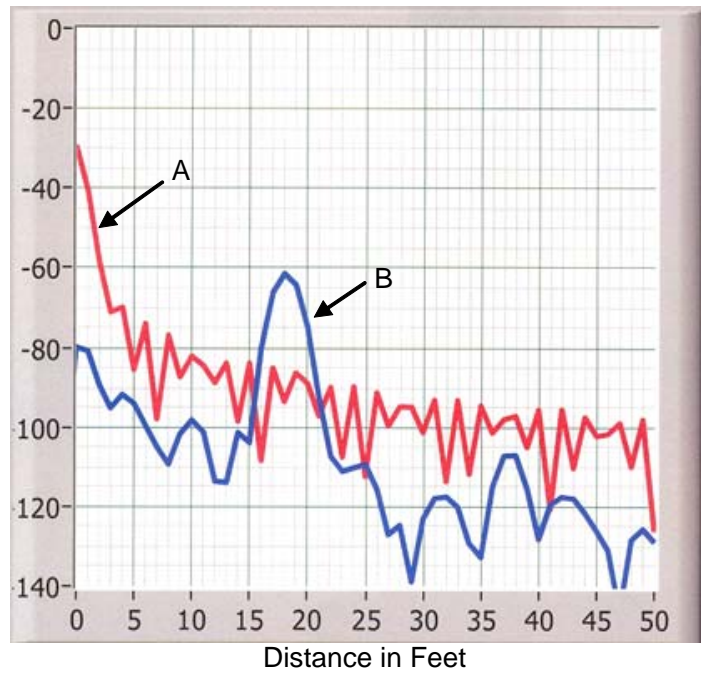

Figure 4-11. Near- (curve A) and far-field (curve B) measured data of a stepped-frequency, continuouswave radar.

In Figure 4-11, Curve A is the near-measurement-mode processed SFCW frequency domain data that have been transformed to the time domain where the wave velocity (v) and round-trip travel 
time $(\tau)$ have determined the distance to the second interface reflection from an abandoned mine void located at 17 feet. Curve B is the far-measurement mode time domain response results from a predistorted FMCW or SFCW radar processed to suppress the first interface reflection and reveal the second weaker reflection from the abandoned mine void.

The zero time-distance ring illustrated in the Curve A response masks the second interface reflected wave. Essentially the radar has been blinded by the reflection from the first interface for a distance of at least 2.5 feet. Although the first interface reflection dominates the nearmeasurement mode response, the near measurement data are useful in determining the electrical conductivity or relative dielectric constant of the first coal layer, which depends on the loss tangent. These data also are used in predistorting the transmitted waveform to correct for the frequency and phase response of the antennas and the changing petrophysics of coal. Thus, the advanced radar design solves two different problems. The predistorted FMCW or SFCWresponse (Curve B) indicates that the stress-fracture-coal-thickness effect has been suppressed, while the weak reflection from the second interface has been emphasized.

The Fourier transform or the algorithm that transforms data from the frequency to the time domain is embedded in the radar electronics field-programmable gate array (FPGA). The frequency domain reflected wave is processed in the FPGA, creating the time-domain representation in Figure 4-11, Curve B. The radar electronics features a microprocessor that downloads the program software defining the digital electronic functions of the radar architecture.

The advanced radar features a software-definable transceiver (SDT) and processing algorithms. The magnitude, frequency, and phase of each frequency component (i.e., sinusoidal waveform) are set by a microprocessor to generate a predistorted waveform. A radar based on a SDT can be programmed to generate phase, frequency, or amplitude-modulated predistorted waveforms. For example, an amplitude-modulated, suppressed-carrier waveform can be generated as shown in Figure 4-12.

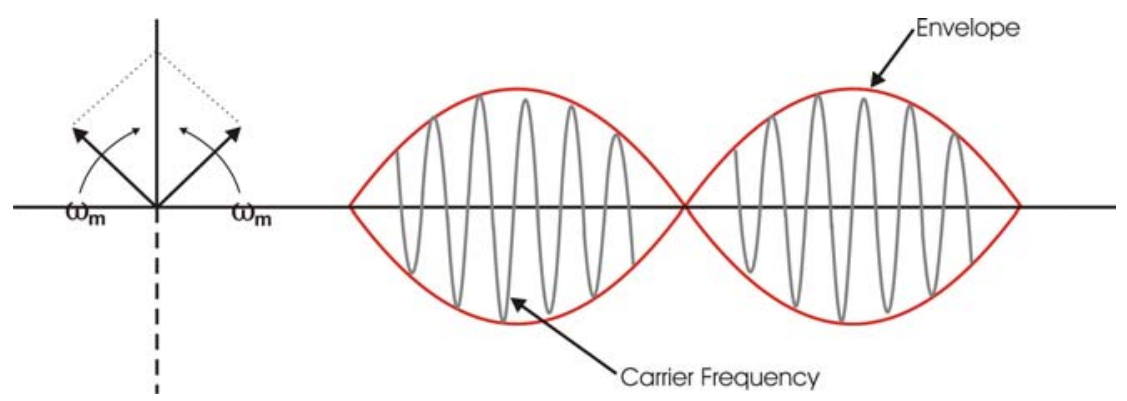

Figure 4-12. Double-sideband, suppressed-carrier waveform.

The SDT radar electronics are shown in Figure 4-13. A prototype of the SDT electronics uses a highly integrated digital printed circuit board (PCB) and a plug-compatible analog PCB (see Figure 4-14). The digital PCB achieves the design requirement of in-application programming (IAP) so that software changes and data acquisition can be achieved in real time. Fieldprogrammable gate arrays (FPGA) are built into the digital PCB. 
Figure 4-13. Software definable transceiver (SDT) radar electronics.

\section{Digital IF Board - DIF (Top)}
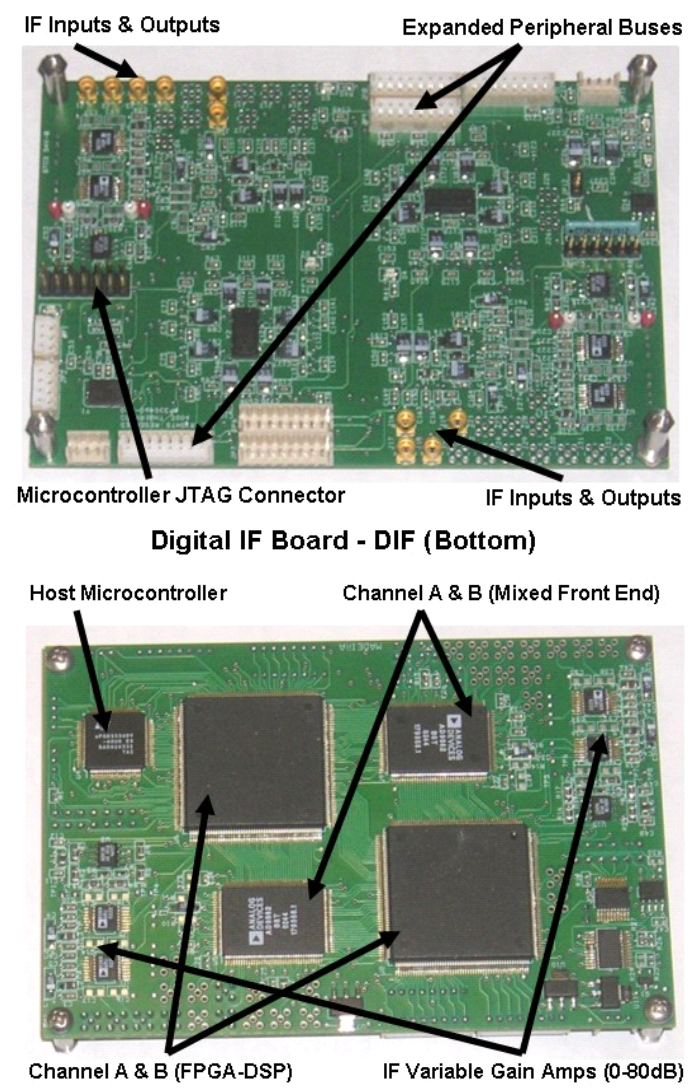

Figure 4-14. Top and bottom surfaces of the very low frequency (VLF) and low-frequency (LF) /medium frequency (MF) band digital SDT printed circuit board.

The digital PCB has two Xilinx ${ }^{2}$ FPGAs, an 8-bit host microcontroller with a 256 kilobyte onchip flash memory, and 12-bit analog-to-digital (A/D) and digital-to-analog (D/A) converters.

\footnotetext{
${ }^{2}$ Xilinx is a trademark of Xilinx, Inc., San Jose, California.
} 
An RS-232 serial communications link and Bluetooth modem also are included in the design. The FPGA logic device replaces tens of standard logic devices and allows functional changes to the peripheral hardware without making any direct changes to the hardware. The combination of these devices allows optimum software and hardware task distribution.

In such a configuration, the microcontroller acts as a host processor, governing the FPGA as a reconfigurable universal interface between the board and external devices. Processing software is downloaded onto the digital PCB from LabView. At startup, the microcontroller downloads to the FPGA. Changes to the microcontroller software or the FPGA configuration require only reprogramming of the microcontroller.

The FPGA's direct digital synthesizer (DDS) or external DDS achieves the predistortion function. Predistortion generally requires the insertion of a compensating frequency response module before the transmitter radio frequency (RF) power amplifier (PA). The predistorter has the inverse response of the PA and antenna so the overall response is linear. Adaptive digital predistortion involves a digital implementation of the predistorter and a feedback loop for adapting to the changes in the response of the PA and antennas.

The two main types of adaptation algorithms are the "blind adaptive" algorithms based on distance-gradient methods and the "polynomial function" algorithms that attempt to directly model the nonlinearities in the radar forward and reverse transmission paths. The analog PCB includes a quadrature up converter, RF power amplifier, directional coupler (i.e., for a monostatic radar), phase locked loop (PLL), or several quad DDSs, and a down converter. A simplified block diagram of the SDT radar, including digital and analog PCBs, is illustrated in Figure 4-15.

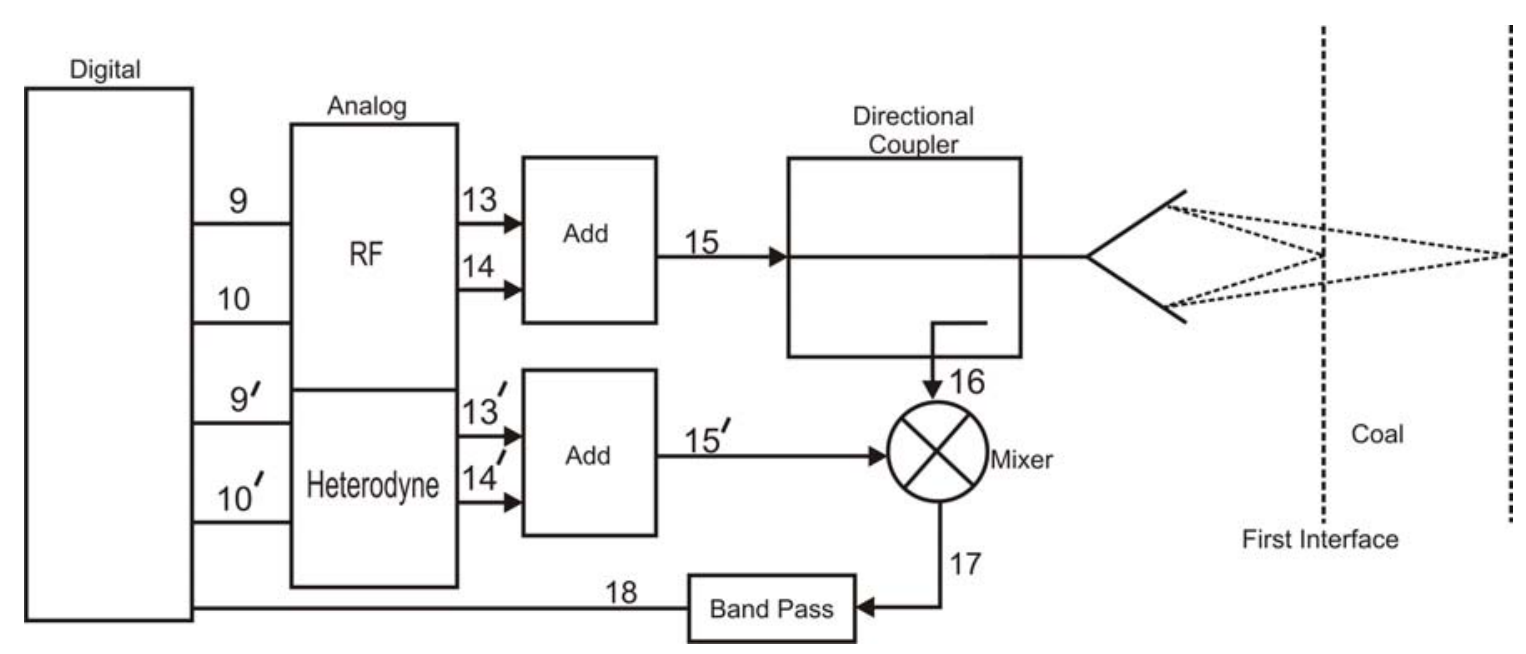

Figure 4-15. Block diagram of a software definable transceiver radar.

A detailed functional block diagram of the SDT is shown in Figure 4-16. The microprocessor on the digital PCB controls the frequency and phase of the digital frequency synthesizers $\omega_{1}, \omega_{2}, \omega_{3}$, and $\omega_{4}$. The step in the CW transmit frequency is determined by the PLL $\omega_{0}$. This PLL is controlled by the microprocessor. 


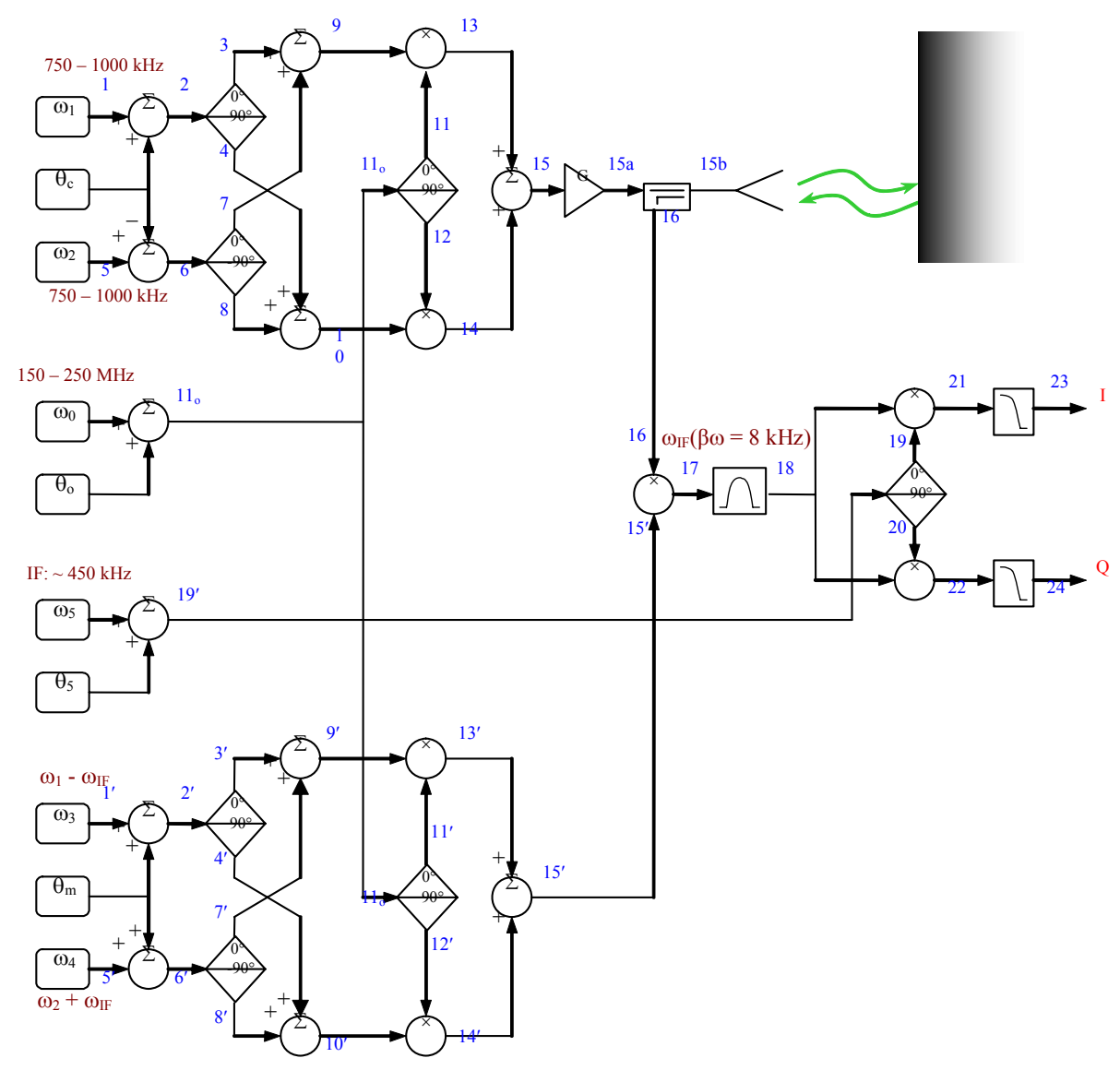

Figure 4-16. Detailed functional block diagram of the software definable transceiver.

The directional coupler recovers the reflected wave. Phase-coherent detection is achieved by mixing the DDS signal with the reflected point signal and bandpass filtering the mixer output signal. An important feature of the phase coherent detection scheme is that the in-phase (I) and quadrature $(\mathrm{Q})$ terms are simultaneously measured in the digital electronics. Simultaneous measurement improves noise immunity.

The measurement of the relative dielectric constant $\left(\varepsilon_{\mathrm{r}}\right)$ of the media and the subsequent detection and range to target (second interface) requires a $90^{\circ}$ phase shift $\left(\theta_{\mathrm{m}}\right)$ in the modulation frequency of the transmitted double sideband waveform. Another unique feature is that the carrier phase $\theta_{c}$ can be set to greatly improve the quality of the transform from the frequency to time domain. The frequency chart shown in Figure 4-17 helps explain the frequency operation of the SDT-driven advanced radar system.

The antenna structures integrated with the SDT radar for radio geophysical applications support transmission of double-sideband $\left(\omega_{\mathrm{o}}-\omega_{1}, \omega_{\mathrm{o}}+\omega_{2}\right)$, suppressed carrier $\left(\omega_{\mathrm{cm}}\right)$ waveforms. Partial suppression of the first interface reflection can be achieved under some conditions with polarized antennas. Additional suppression of approximately $20 \mathrm{~dB}$ can be achieved with circularly polarized antennas. The polarization of the transmitter (TX) and receiver (RX) antennas depends on the distance to the first interface and the presence of in-phase surface waves. In some 
instances, the antenna pair must be oppositely polarized because of the lateral surface wave inphase transmission from the TX to RX antennas.

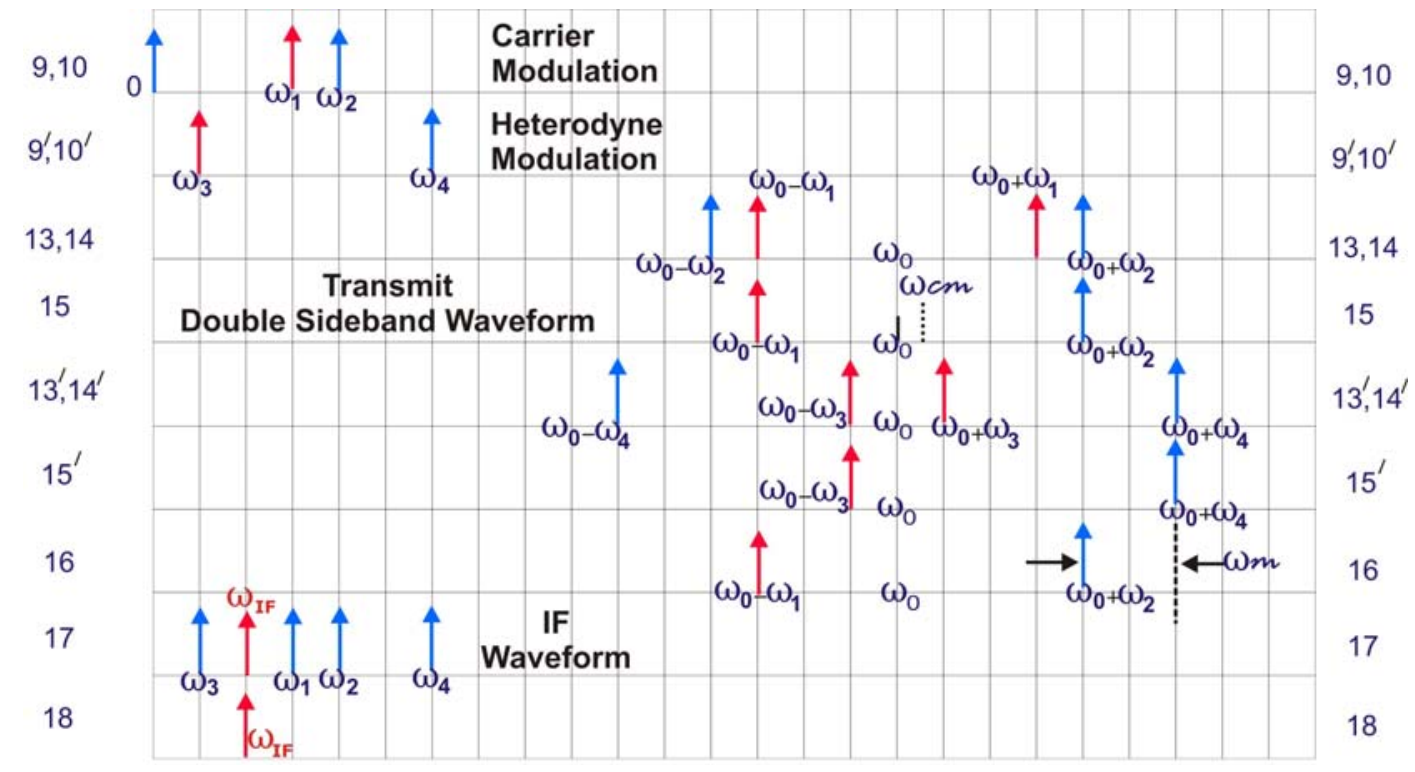

Figure 4-17. Frequency chart for the software definable transceiver.

\subsection{Prototype Mid-Seam Guidance Tool}

During the course of the development of the drill string radar technology, a simplified tool was conceived that has the capability of maintaining a horizontal well in an optimal manner in certain formations to maximize hydrocarbon recovery. This tool, known as a mid-seam guidance tool, allows a horizontal wellbore to be placed equally distant from the upper and lower boundary rock surrounding the oil-bearing strata.

The simplicity of the mid-seam guidance tool will reduce the problems associated with the use of currently available complex implied boundary determination methods. For more demanding applications, a true radar (see Section 4.3) would be employed to make a direct measure of the boundary location in real time in horizontal drilling applications as opposed to implied methods relying on extensive commutations.

\subsubsection{Electromagnetic Gradiometer Background}

The mid-seam guidance tool capitalizes on expertise development for other applications in the form of an electromagnetic (EM) gradiometer system, which permits the detection of features in lossy soils in real time. The gradiometer measures the gradient of the horizontal magnetic field strength. A vertical magnetic dipole (VMD) transmit antenna is used to illuminate the intended features. The VMD transmit antenna has horizontal electric fields that optimally couple to horizontal subsurface features. Primary EM waves interact with subsurface structures to create secondary waves that are detectable. The gradiometer technology can generate subsurface geophysical imaging capabilities with greater sensitivity, range, and flexibility than alternative 
instrumentation that measures the absolute magnetic field rather than the gradient of the magnetic field (see Figure 4-18).

In Figure 4-18, the EM gradiometer response over a subsurface void is shown. The response illustrates the limitation of EM measurement methods that measure the total magnetic field. Because the primary field is much larger than the secondary field, the total field changes by only a few percent when the survey line crosses over a significant geologic anomaly, whereas the gradient field changes by a significant amount. Instrumentation that measures total fields would exhibit poor resolution. However, the resolution of the EM gradiometer technology is very high, which demonstrates that measuring resolution is not always related to wavelength.

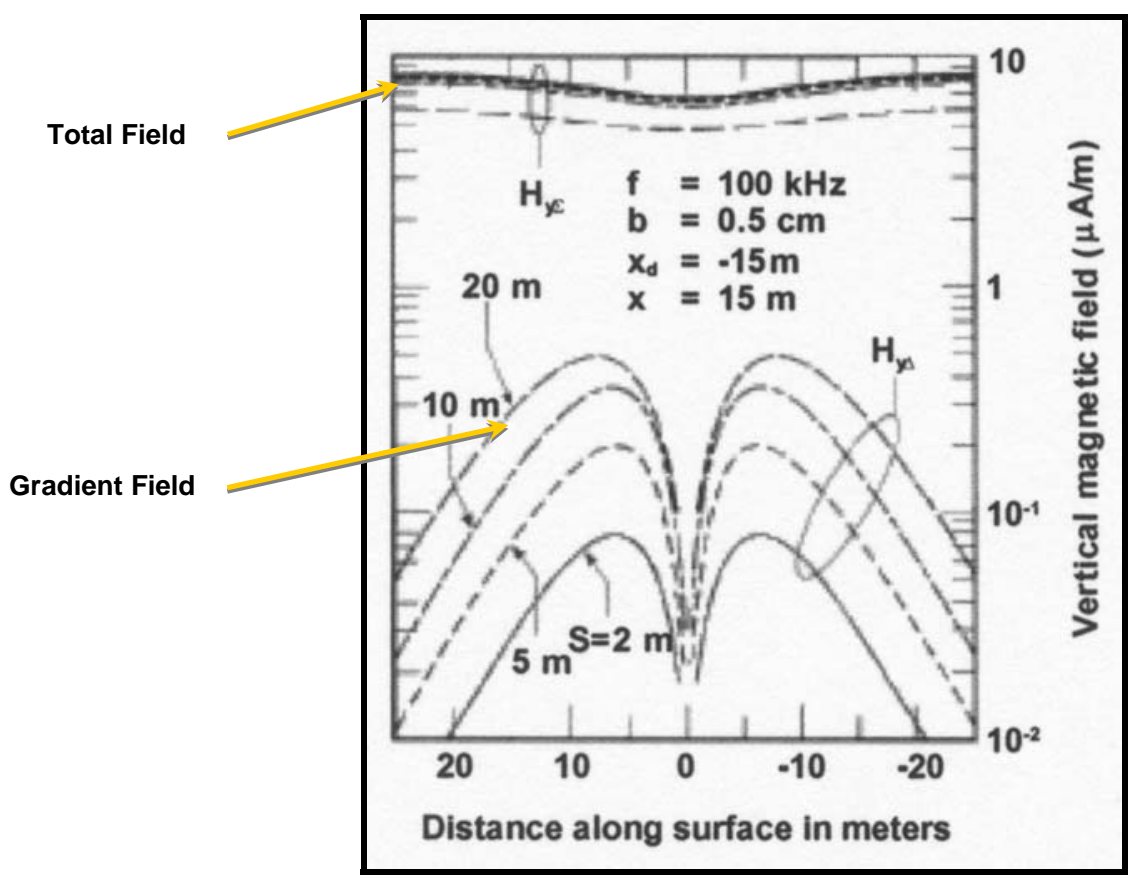

Figure 4-18. Surface electromagnetic gradiometer response over a subsurface void.

\subsubsection{Experimental Design of the Prototype Mid-Seam Guidance Tool}

By employing low-frequency loop antennas, Stolar's electromagnetic gradiometer technology measures the gradient of the received signals and reduces the effects of the primary wave in the process. A diagram of the gradiometer system in a horizontal borehole is shown in Figure 4-19, where the two receiver (i.e., RX1 and RX2) antennas are the same distance from the transmitter (TX) antenna. The physical separation between the transmitter and receiver antennas also reduces the effects of the invasion zone created by the borehole drilling fluid and emphasizes the effects of the bounding rocks.

In order to determine the direction of the reflected waves in a three-dimensional imaging scheme, each RX antenna measures all three orthogonal vector components of the magnetic field intensity. These measurements are accomplished by using three loop antenna orientations as shown in Figure 4-20. The placement of antenna pairs also allows the determination of the gradient of the magnetic field intensity. The main transmitting and receiving loop antennas are 
$\mathrm{x}$-directed horizontal magnetic dipoles (HMD-x), wrapped around the axis of the drill rod. Not only do the y-directed horizontal magnetic dipoles (HMD-y) and the vertical magnetic dipoles (VMD) provide information regarding the direction of the reflected waves, but these dipoles also act as bucking coils by reducing the effects of the primary wave for the main HMD-x receiver antennas.

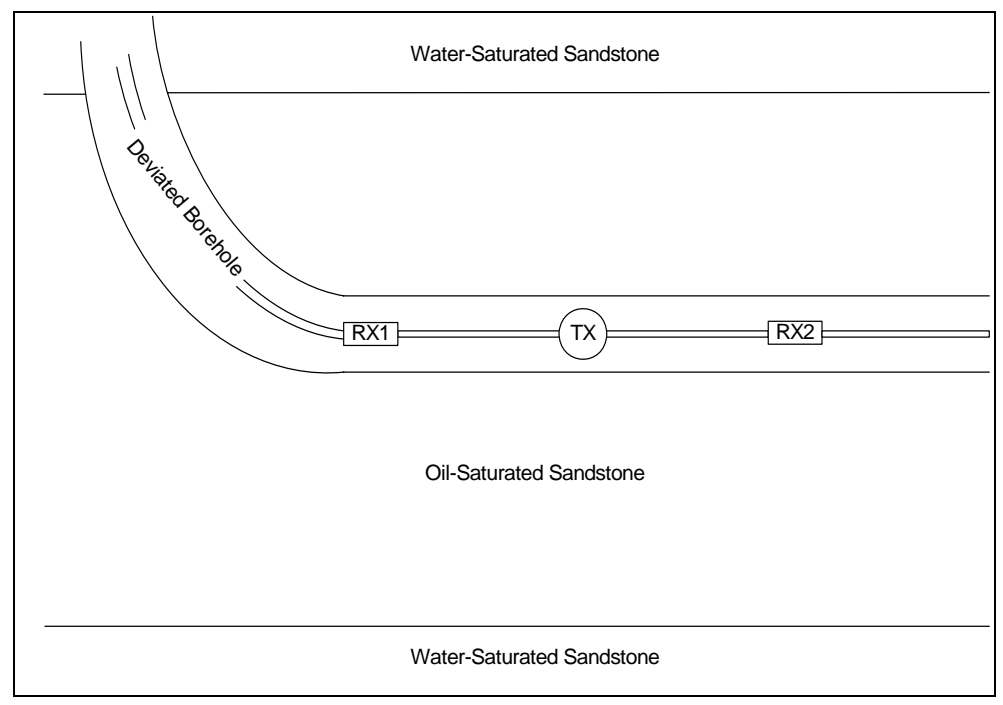

Figure 4-19. Prototype mid-seam guidance in a horizontal borehole in an oil reservoir.

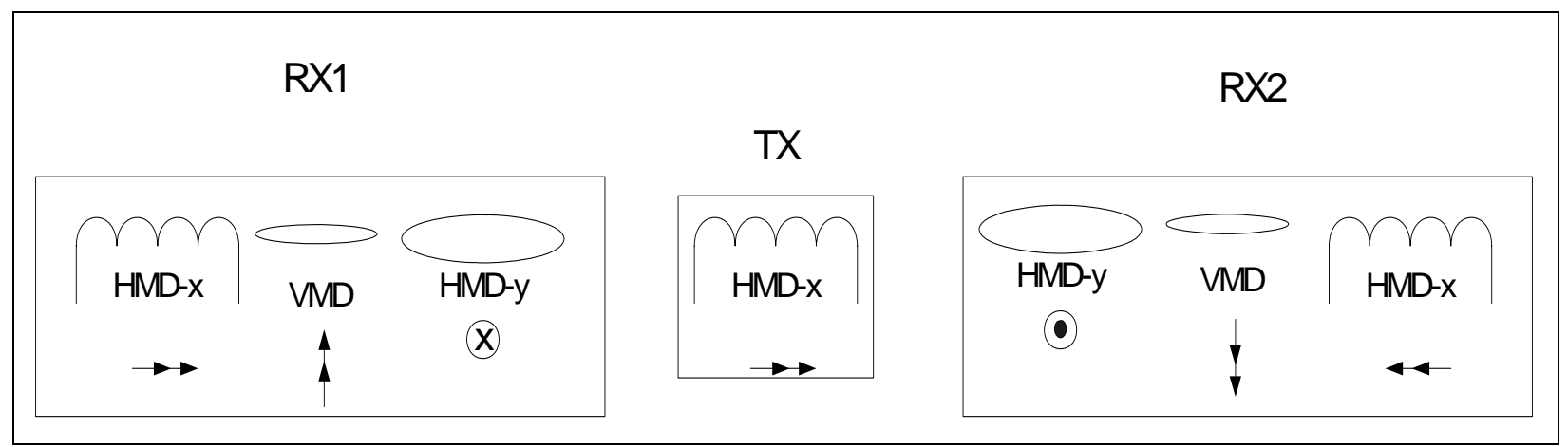

Figure 4-20. Prototype mid-seam guidance tool antenna orientations.

In addition to keeping track of multiple instantaneous measurements for detecting the boundaries of an oil reservoir, the gradiometer system incorporates the information about the orientation of the tool and the borehole. Comparison of the signals from the matched pair of receivers allows determination of whether the tool is in mid seam, or if one antenna but not the other is approaching the boundary rocks of a hydrocarbon reservoir.

An initial prototype of the mid-seam guidance tool was constructed using two loop antennas fabricated identically using 690 turns of 26-AWG wire. The loops forming the antennas were wrapped around a Garolite tube, which is a paper-based laminate with a phenolic resin binder, of 0.75-inch diameter. The antenna section was 12.25 inches long. Each antenna required a 50- 
picofarad tuning capacitor to achieve an operating frequency of about $860 \mathrm{kHz}$ in air. The basic construction features of the test mid-seam guidance tool are shown in Figure 4-21.

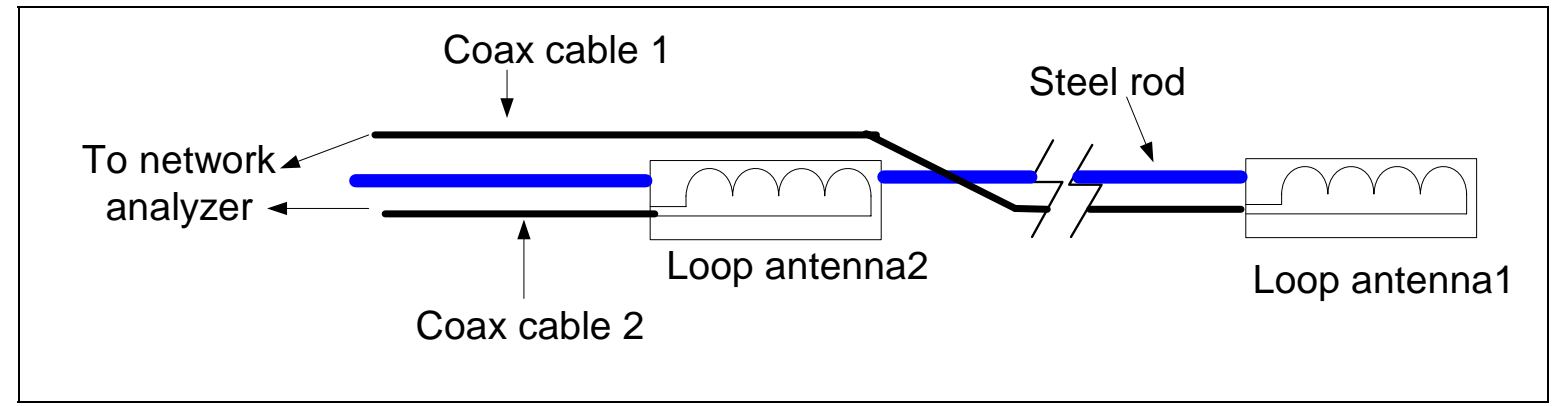

Figure 4-21. Construction features of the prototype mid-seam guidance tool for testing in a deviated borehole to track an air-soil boundary.

\subsubsection{Experimental Results for the Prototype Mid-Seam Guidance Tool}

An experimental facility was constructed to test the feasibility of using a mid-seam guidance tool to detect and track an air-soil boundary (see Figure 4-22). This facility was designed to simulate the difference in electrical properties between the oil-saturated rocks and typical boundary formations in a hydrocarbon reservoir. Two deviated boreholes were drilled: one with an 11degree angle and the other with a 15-degree angle to the air-soil boundary. The borehole dimensions allowed the insertion of 1-inch O.D. tools.

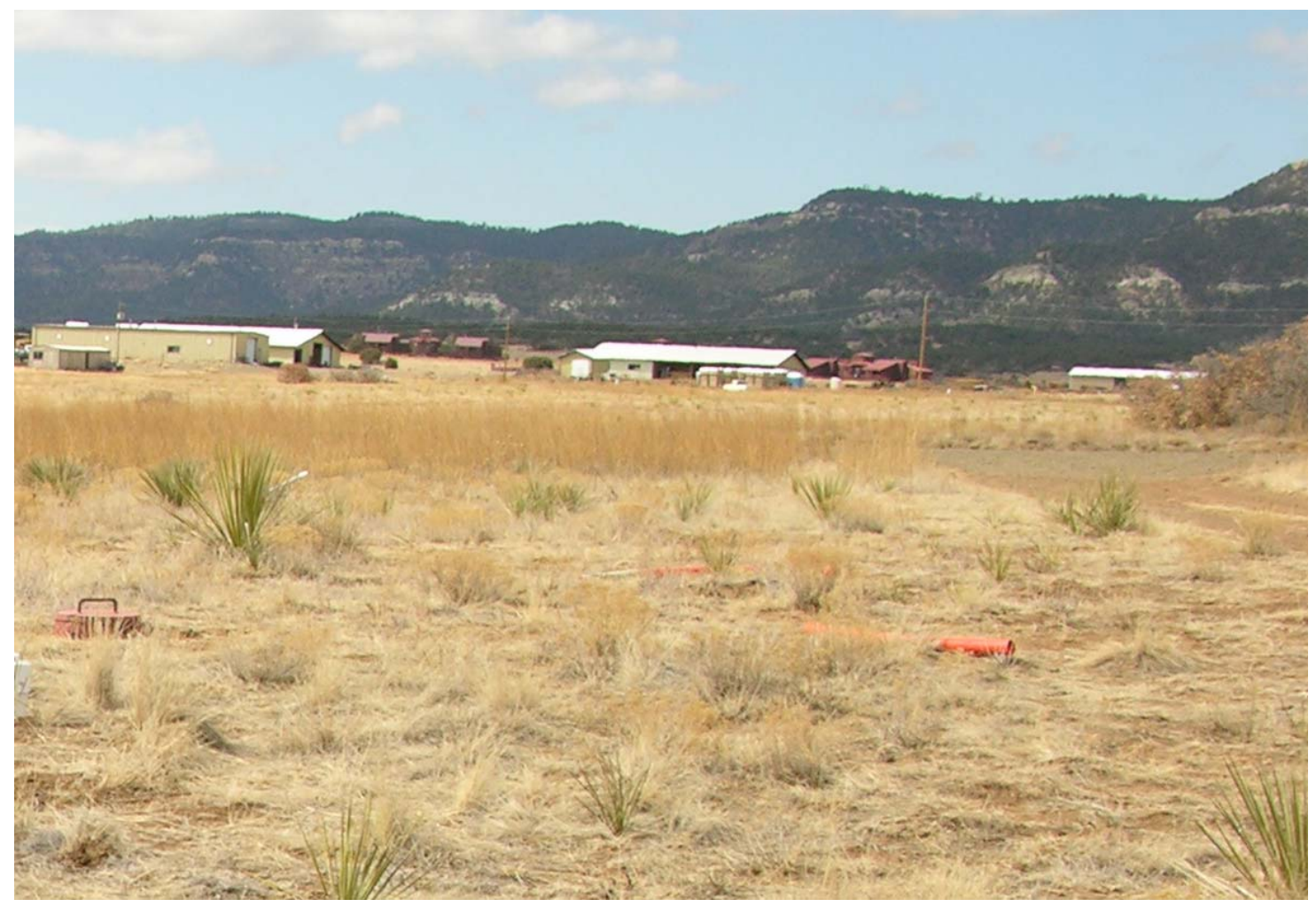

Figure 4-22. Location of the deviated test boreholes for tracking an air-soil boundary. 
The electrical properties of air and soil are vastly different. At amplitude modulation (AM) broadcast frequencies, the ground resistivity of the continental U.S. has been documented by Keller and Frischknecht [11]. In northern New Mexico where the test site is located, the soil resistivity value is relatively low (i.e., 50 to $500 \mathrm{ohm}-\mathrm{m}$ ), much the same as the resistivity of oilsaturated rocks in a typical oil reservoir. If the mid-seam guidance tool could not detect and track an air-soil boundary, the tool would be unable to detect reflections from a weaker reflecting boundary like the bounding rock of a hydrocarbon reservoir.

For the initial test, two loop antennas were connected to the two ports of an Agilent 8753ES vector network analyzer. The network analyzer can measure the reflection coefficient (or equivalently the input impedance) of individual ports and the transmission coefficient between the two ports across a band of frequencies.

The two axially wrapped loop antennas were connected to each other via either a 10-foot or 5foot long steel rod and inserted in the test borehole with an 11-degree angle to the air-soil interface. The coaxial cable of the antenna that was farther inside the test hole had to cross over the other antenna, which was a shortcoming of the initial test setup. However, this condition is easily corrected with a more advanced design.

The Agilent network analyzer was used to measure the reflection coefficient from each loop antenna, as well as the transmission between the antennas. The real and imaginary components of the measured reflection coefficient of the individual antennas are shown in Figures 4-23 and $4-24$, respectively. The measurements were taken with the antennas lying on the ground and with the antennas inside the test borehole for tracking the air-soil boundary. The transmission loss between the two test antennas is shown in Figure 4-25.

When the antennas were placed above ground, their resonant frequency was higher than when the antennas were placed inside the test hole. The reflection coefficients do not seem to indicate a pattern. However, the transmission loss between the two antennas increases as the mid-seam guidance tool was pushed deeper inside the hole, which indicates that the air-soil boundary was constructively, and predictably, affecting the transmission between the two loop antennas.

There is an anomaly in the reflection of antenna 2 when at a depth was 1.1 feet (see the brown trace in Figure 4-24). This anomaly appears the transmission losses as well (see Figure 4-25). Electrical and geological noise may have corrupted the data to a point that some data points must be disregarded, which probably explains this observed anomaly.

The results of a second set of transmission tests are graphed in Figures 4-26 and 4-27 for the deviated boreholes that had an 11-degree and a 15-degree angle of entry in the soil with respect to the air-soil boundary. In order to test the impact of separation distance, the antennas were separated by either five feet or 10 feet.

As the antennas were pushed further inside the deviated boreholes, the transmission loss between the two antennas generally increased. This result is particularly evident when the separation between the transmitter and receiver antennas was five feet as the antennas were pushed inside 
the 15-degree-deviated borehole (Figure 4-27). This trend was not true in all situations, which can be attributed to geological and electrical noise in the test environment. To avoid inaccurate predictions, the measured data must be used based on their current value and their history in conjunction with the orientation information. Spatial diversity of the antennas gives an added degree of confidence in the measurements.

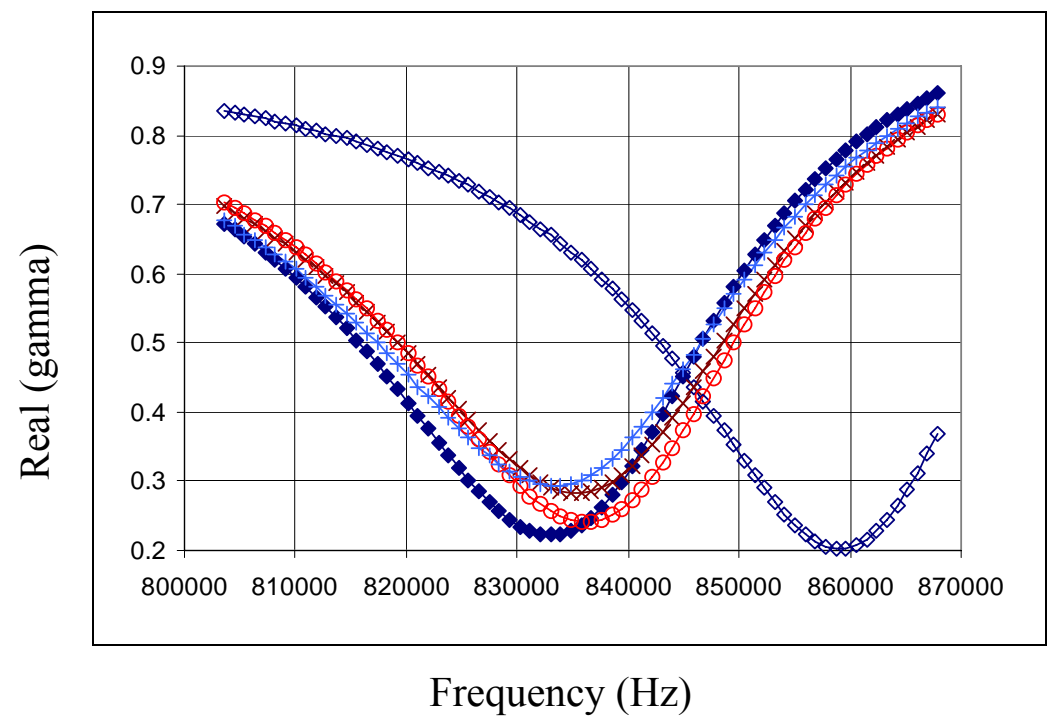

(a)

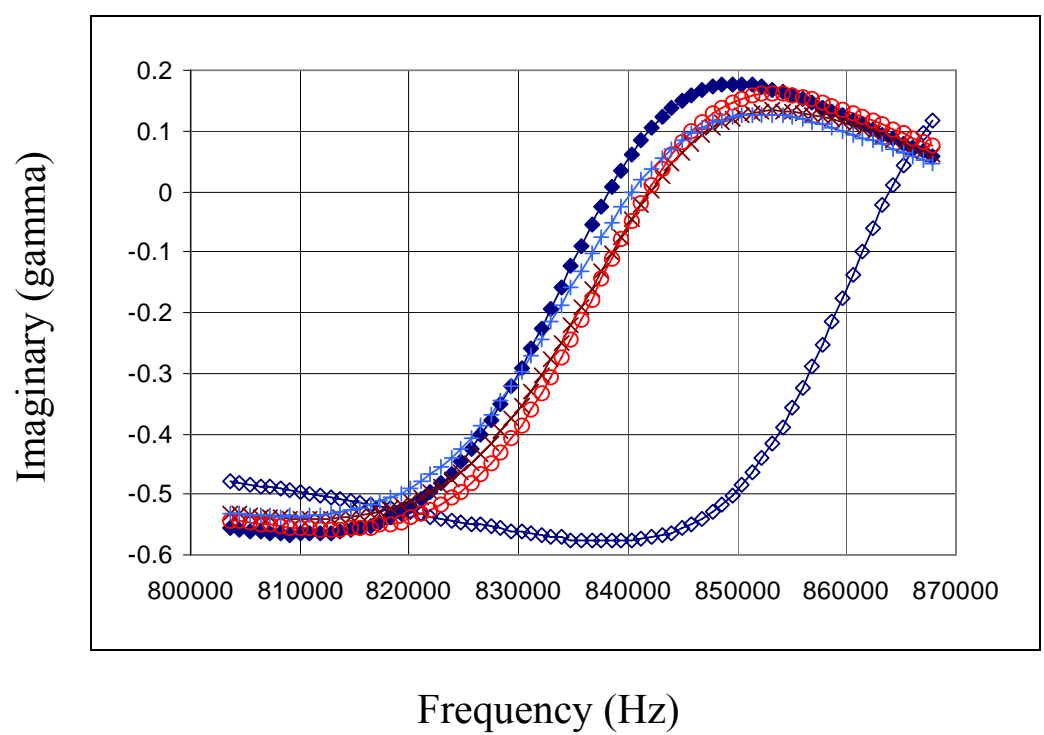

(b)

Figure 4-23. Reflection from antenna 1 in the deviated test borehole: (a) real part of the reflection coefficient and (b) imaginary part of the reflection coefficient. The blue traces with unfilled diamonds correspond to antenna 1 on the ground at zero depth. The blue traces with filled diamonds, the brown traces with $x$ markers, the red traces with o markers, and the blue traces with + markers correspond to antenna 1 at a depths of 1.9, 3.0, 4.3, and $5.2 \mathrm{feet}$, respectively. 


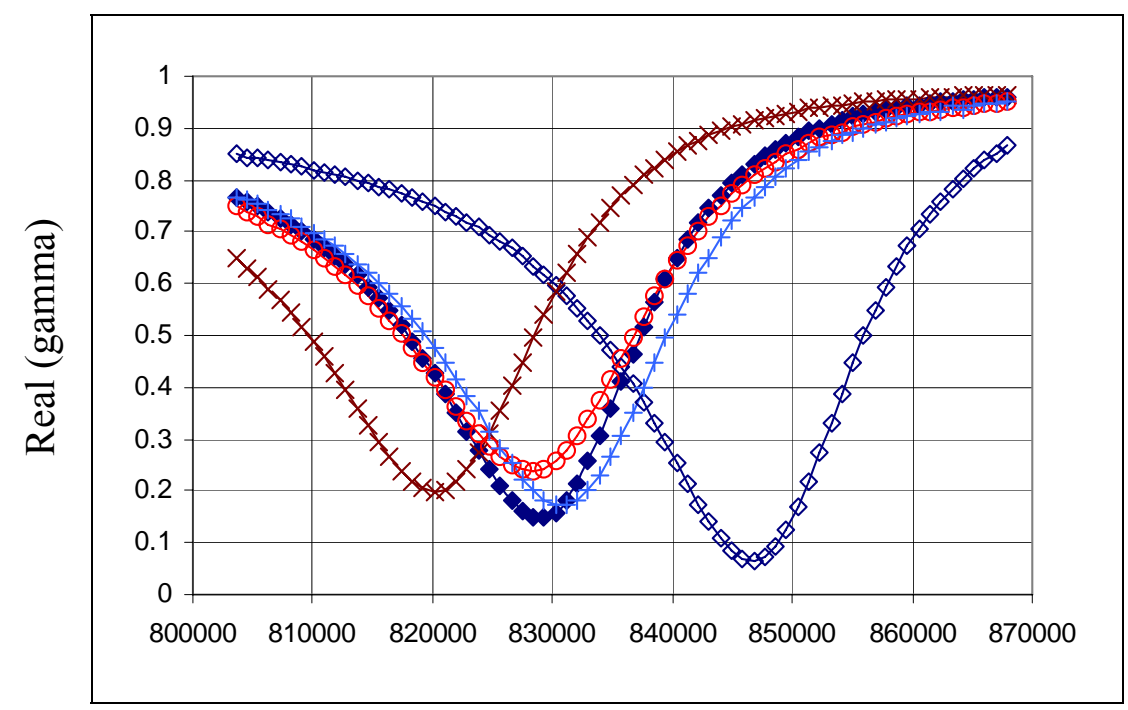

Frequency $(\mathrm{Hz})$

(a)

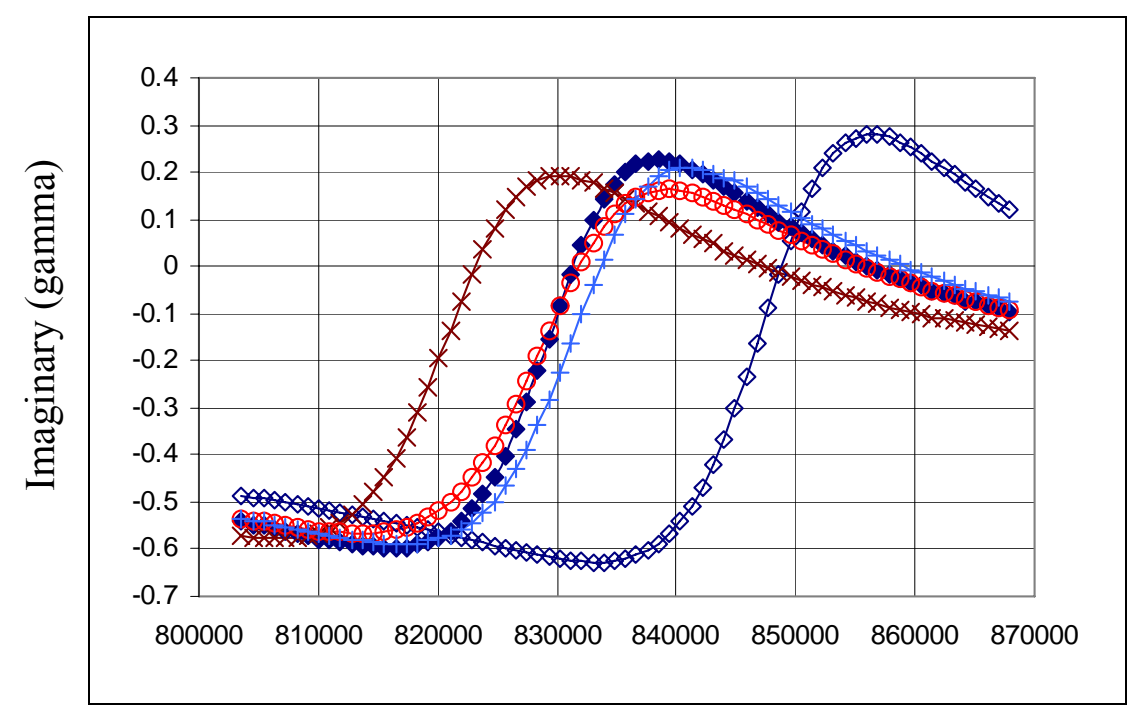

Frequency $(\mathrm{Hz})$

(b)

Figure 4-24. Reflection from antenna 2 in the deviated test borehole: (a) real part of reflection coefficient and (b) imaginary part of reflection coefficient. The blue traces with unfilled diamonds correspond to antenna 2 on the ground at zero depth. The blue with filled diamonds, the brown traces with $x$ markers, the red traces with o markers, and the blue traces with + markers correspond to antenna 2 at depths of $0.1,1.1,2.3$, and 3.2 feet, respectively. 


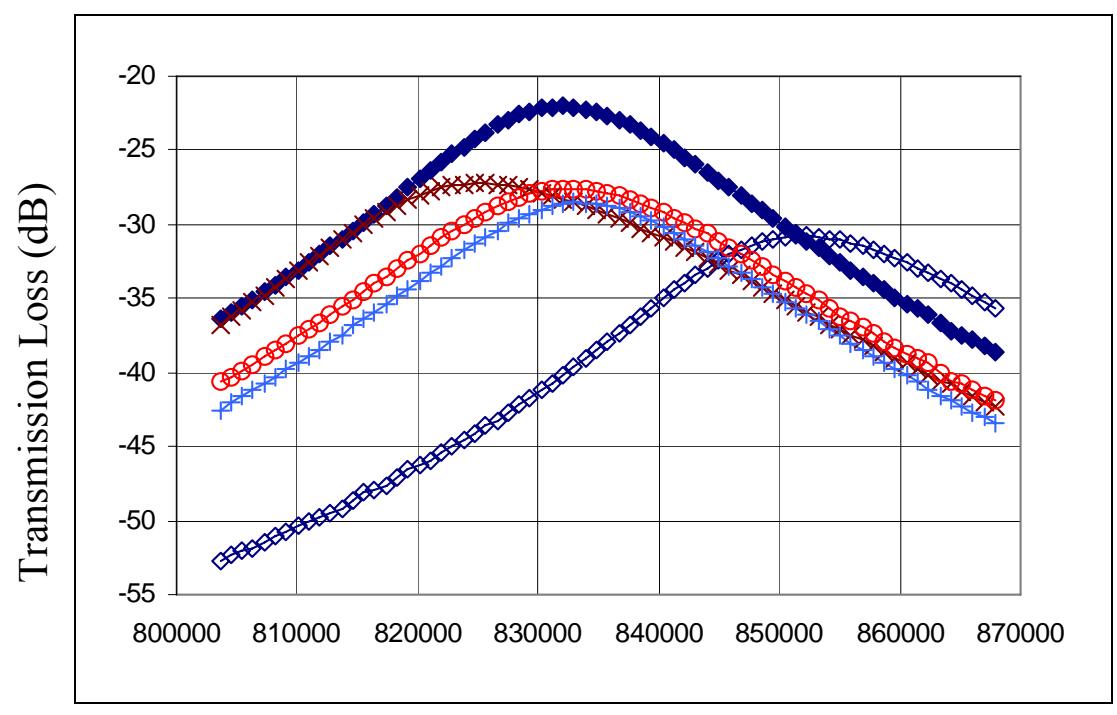

Frequency $(\mathrm{Hz})$

Figure 4-25. Transmission loss between the two antennas in the deviated test borehole. The blue with unfilled diamonds corresponds to both antennas on the ground at zero depth. The blue trace with filled diamonds corresponds to antenna 1 at a depth of 1.9 feet and antenna 2 at a depth 0.1 feet. The brown trace with $x$ markers corresponds to antenna 1 at a depth of 3 feet and antenna 2 at a depth of 1.1 feet. The red trace with o markers corresponds to antenna 1 at a depth of 4.2 feet and antenna 2 at a depth 2.3 feet. The blue trace with + markers corresponds to antenna 1 at a depth 5.2 feet and antenna 2 at a depth 3.2 feet.

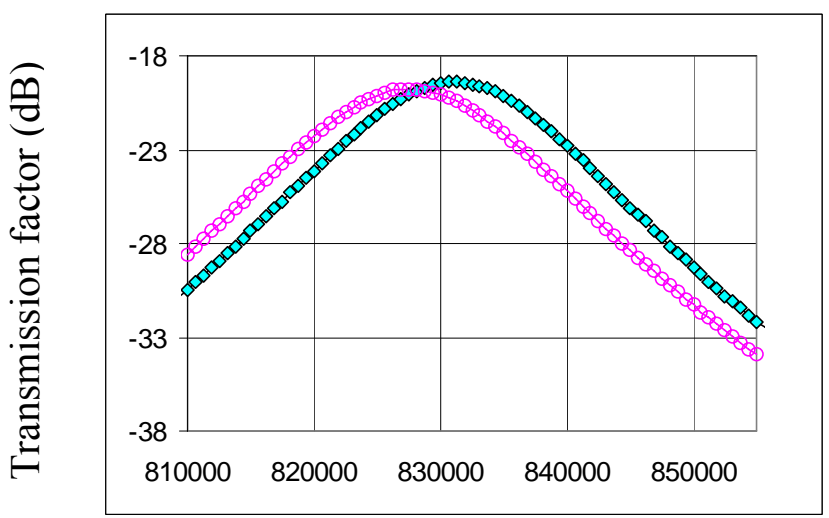

Frequency $(\mathrm{Hz})$

(a)

Figure 4-26. Measured transmission results with a transmitter-receiver pair of tuned loop antennas in the 11-degree deviated borehole test site. The receivers were either 10 feet (pink curves with circle markers) or 5 feet (turquoise curves with diamond markers) ahead of the transmitter in the borehole. The transmitter was (a) 0.1 feet, (b) 3 feet, (c) 6 feet, (d) 9 feet, (e) 12 feet, (f) 15 feet, (g) 18 feet, (h) 21 feet, (i) 24 feet, and (j) 27 feet inside the deviated borehole. 


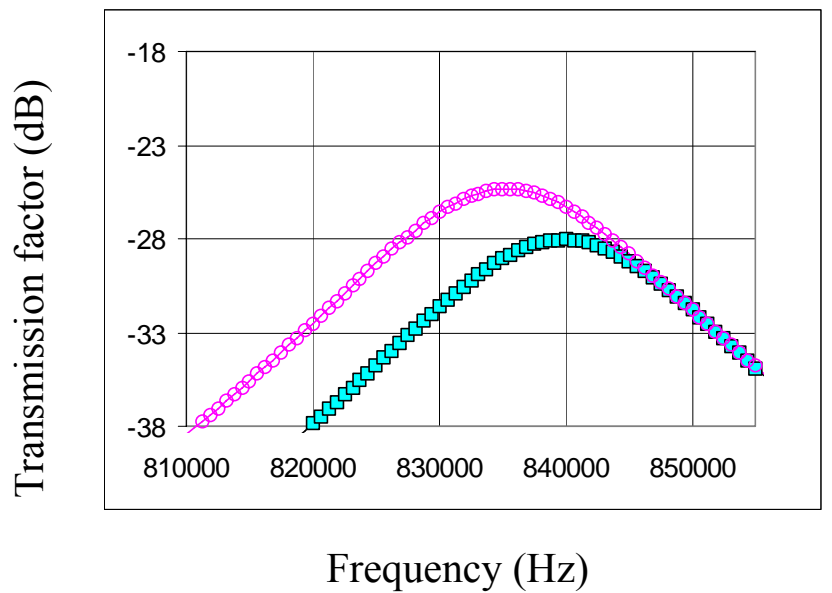

(b)

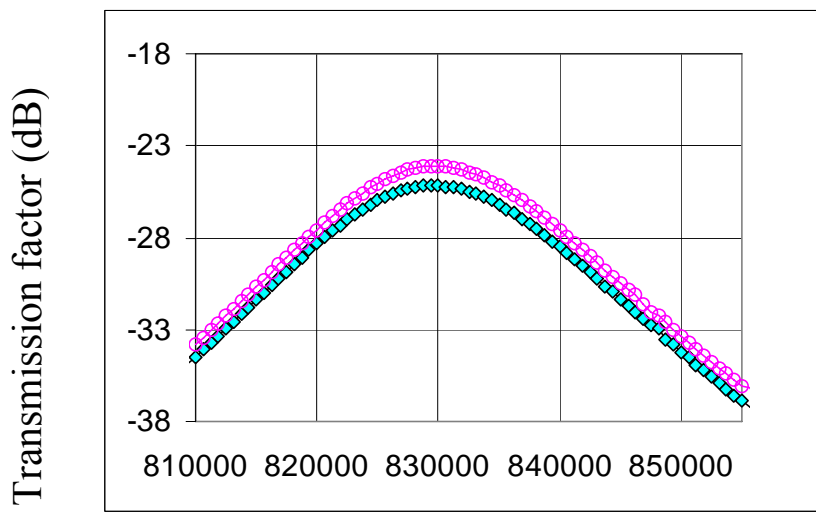

Frequency $(\mathrm{Hz})$

(c)

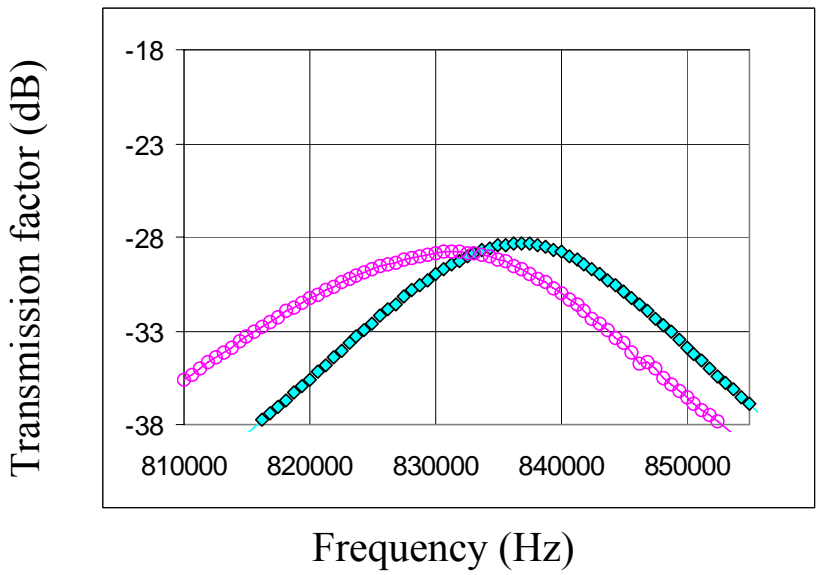

(d)

Figure 4-26. Measured transmission results with a transmitter-receiver pair of tuned loop antennas in the 11-degree deviated borehole test site. The receivers were either 10 feet (pink curves with circle markers) or 5 feet (turquoise curves with diamond markers) ahead of the transmitter in the borehole. The transmitter was (a) 0.1 feet, (b) 3 feet, (c) 6 feet, (d) 9 feet, (e) 12 feet, (f) 15 feet, (g) 18 feet, (h) 21 feet, (i) 24 feet, and (j) 27 feet inside the deviated borehole. 


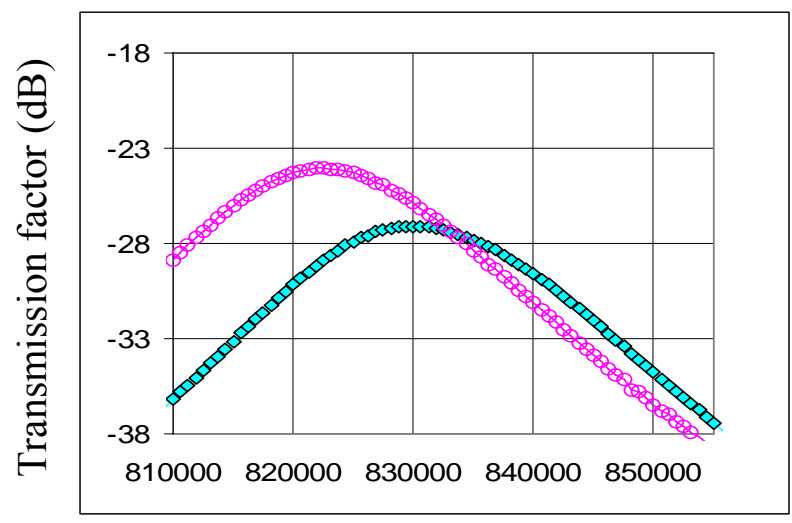

Frequency $(\mathrm{Hz})$

(e)

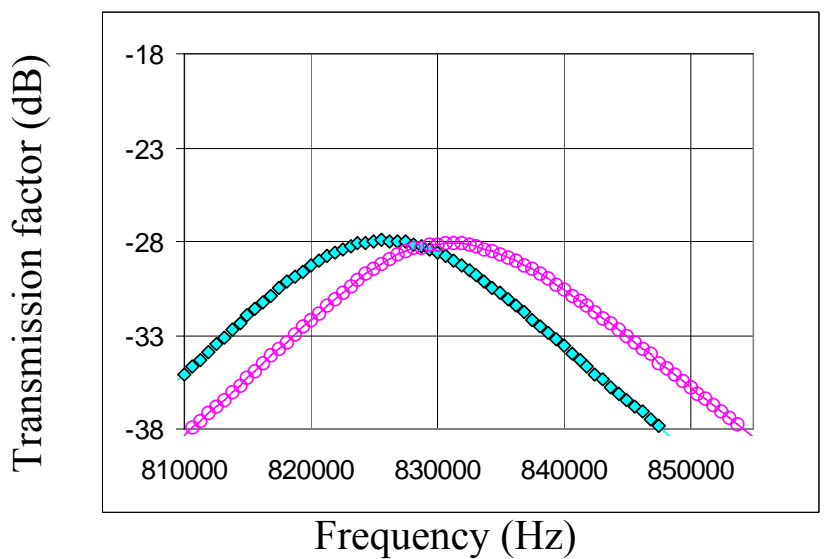

(f)

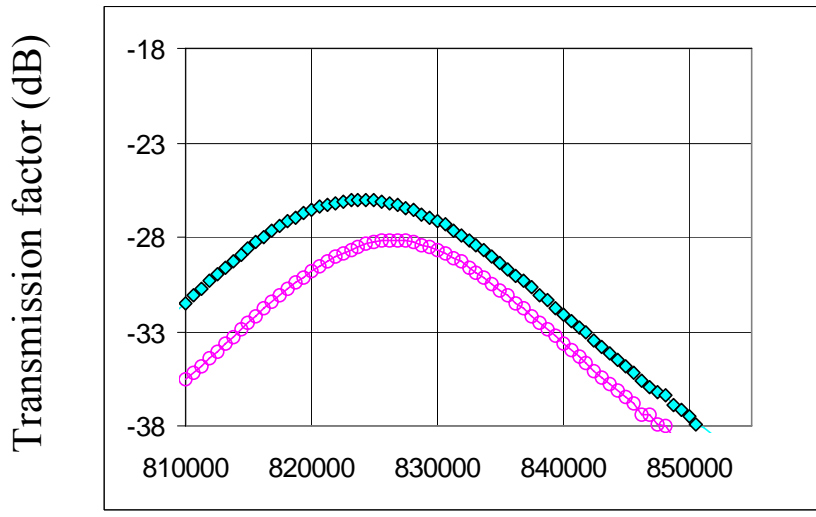

Frequency $(\mathrm{Hz})$

$(\mathrm{g})$

Figure 4-26. Measured transmission results with a transmitter-receiver pair of tuned loop antennas in the 11-degree deviated borehole test site. The receivers were either 10 feet (pink curves with circle markers) or 5 feet (turquoise curves with diamond markers) ahead of the transmitter in the borehole. The transmitter was (a) 0.1 feet, (b) 3 feet, (c) 6 feet, (d) 9 feet, (e) 12 feet, (f) 15 feet, (g) 18 feet, (h) 21 feet, (i) 24 feet, and (j) 27 feet inside the deviated borehole. 


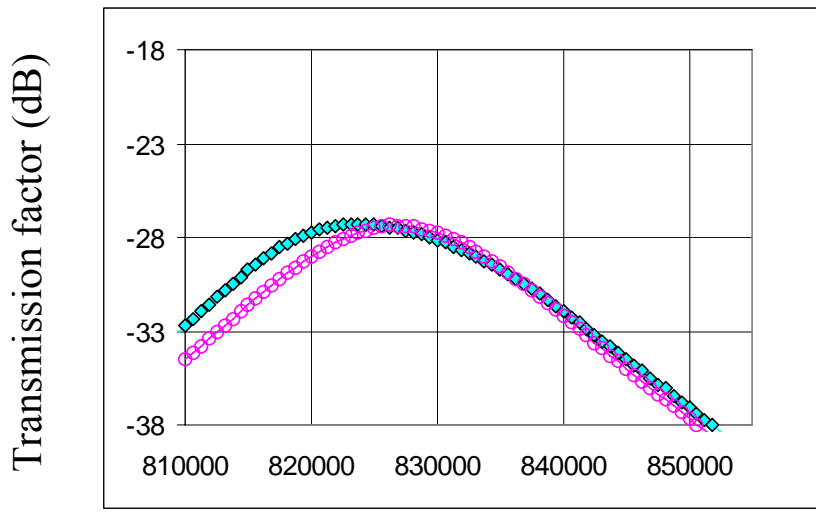

Frequency $(\mathrm{Hz})$

(h)

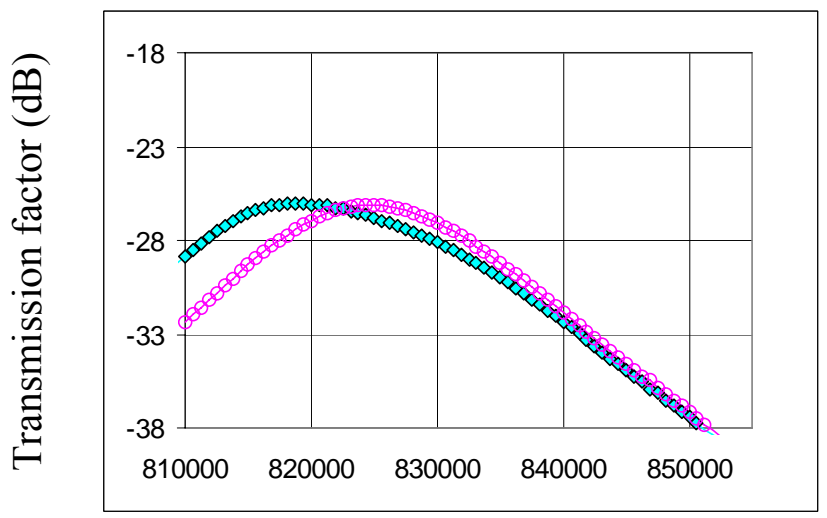

Frequency $(\mathrm{Hz})$

(i)

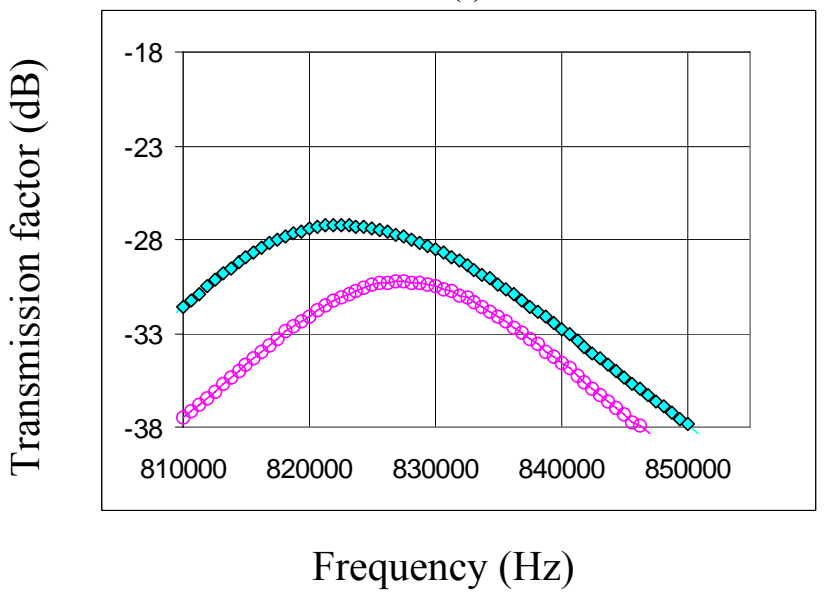

(j)

Figure 4-26. Measured transmission results with a transmitter-receiver pair of tuned loop antennas in the 11-degree deviated borehole test site. The receivers were either 10 feet (pink curves with circle markers) or 5 feet (turquoise curves with diamond markers) ahead of the transmitter in the borehole. The transmitter was (a) 0.1 feet, (b) 3 feet, (c) 6 feet, (d) 9 feet, (e) 12 feet, (f) 15 feet, (g) 18 feet, (h) 21 feet, (i) 24 feet, and (j) 27 feet inside the deviated borehole. 


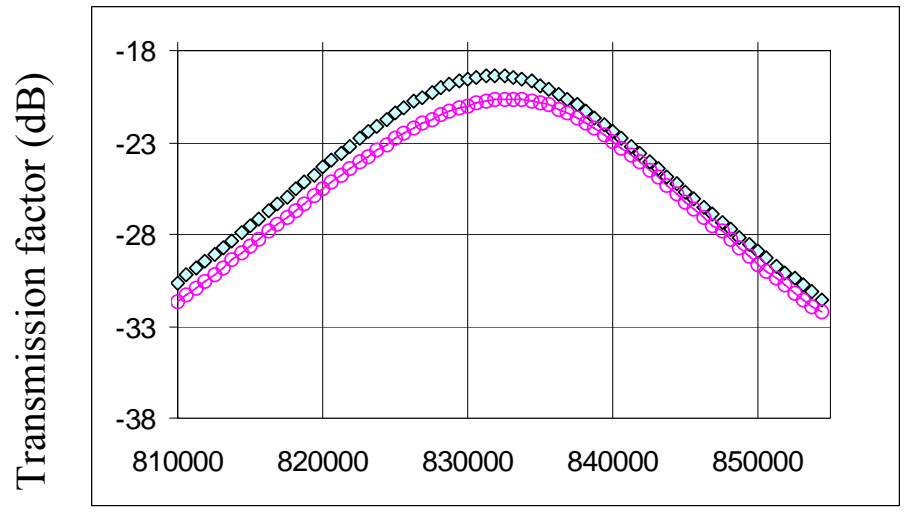

Frequency $(\mathrm{Hz})$

(a)

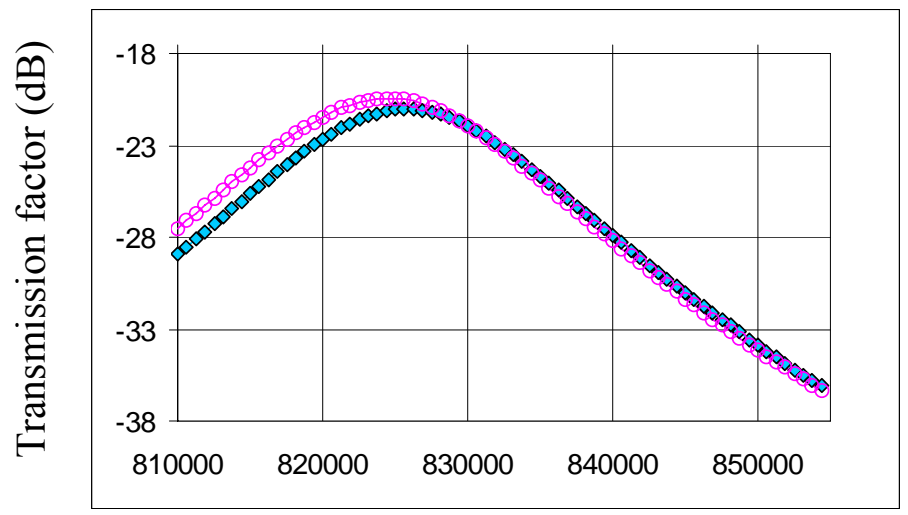

Frequency $(\mathrm{Hz})$

(b)

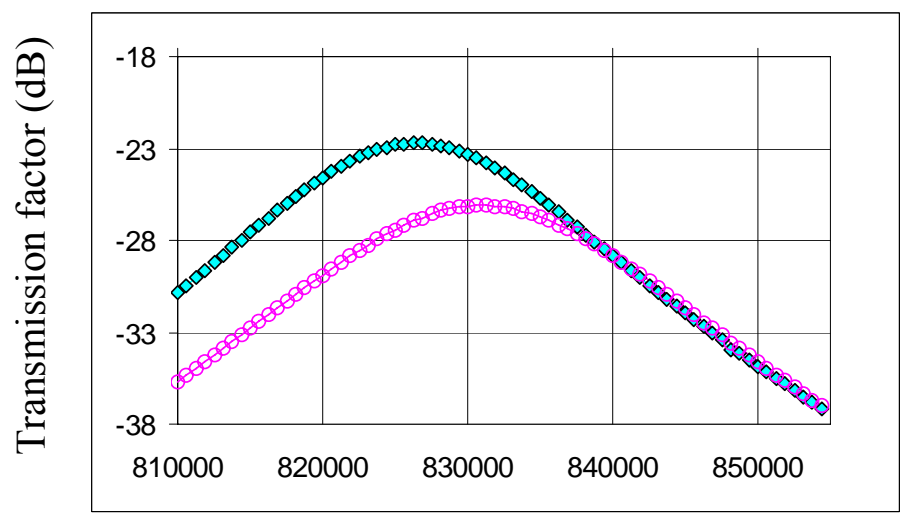

Frequency $(\mathrm{Hz})$

(c)

Figure 4-27. Measured transmission results with a transmitter-receiver pair of tuned loop antennas in the 15-degree deviated borehole test site. The receivers were either 10 feet (pink curves with circle markers) or 5 feet (turquoise curves with diamond markers) ahead of the transmitter in the borehole. The transmitter was (a) 0.1 feet, (b) 3 feet, (c) 6 feet, (d) 9 feet, (e) 12 feet, (f) 18 feet, (g) 21 feet, (h) 24 feet, and (i) 27 feet inside the deviated borehole. 


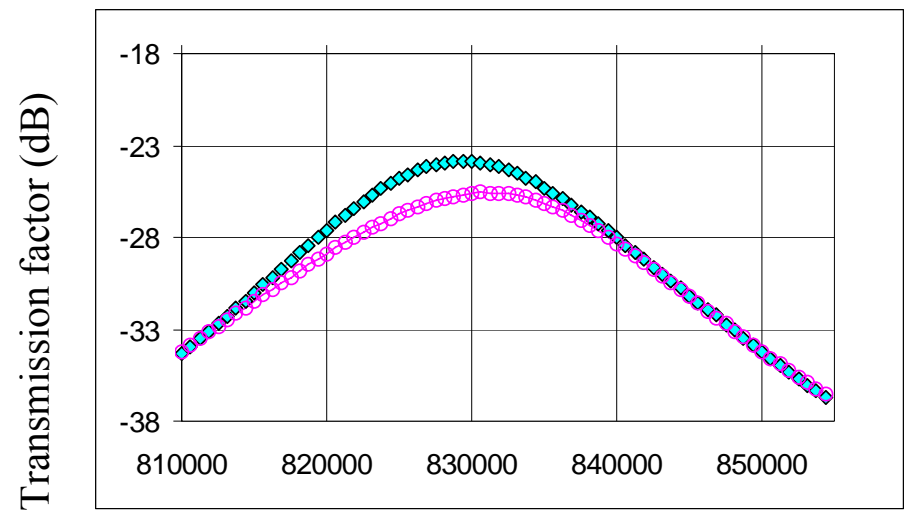

Frequency $(\mathrm{Hz})$

(d)

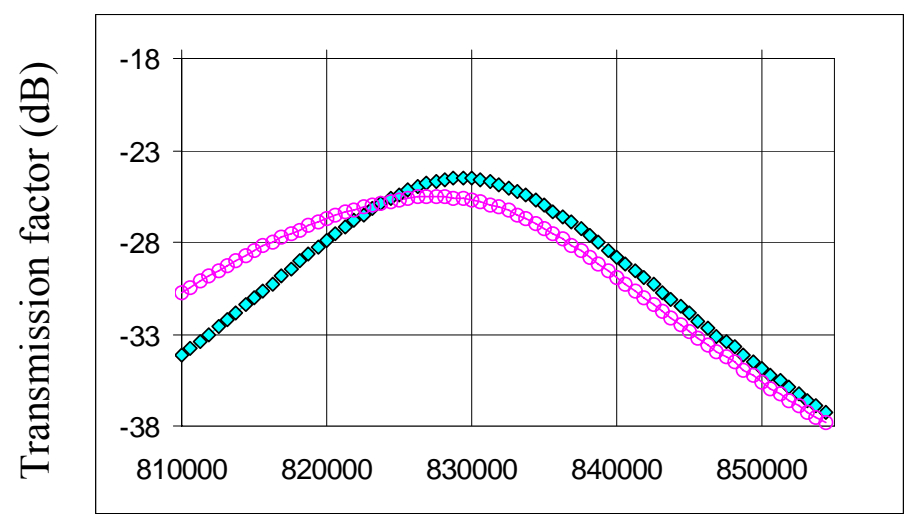

Frequency $(\mathrm{Hz})$

(e)

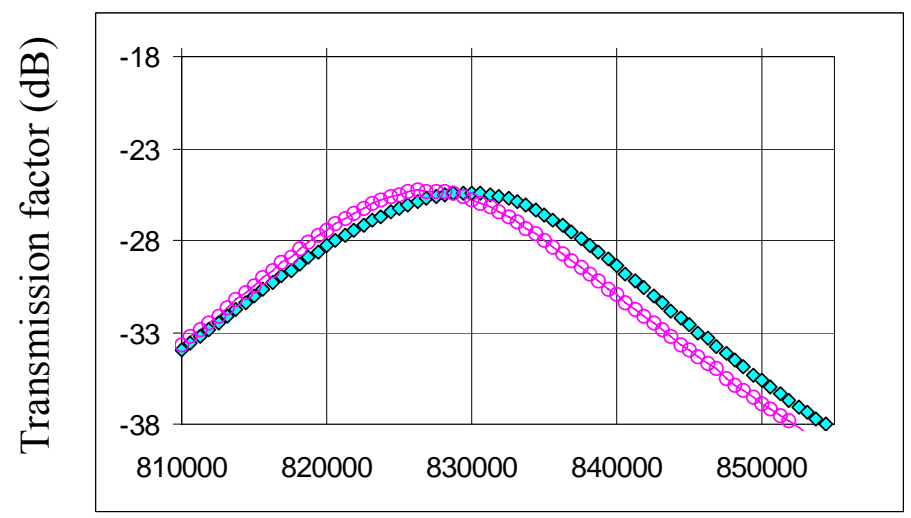

Frequency $(\mathrm{Hz})$

(f)

Figure 4-27. Measured transmission results with a transmitter-receiver pair of tuned loop antennas in the 15-degree deviated borehole test site. The receivers were either $3 \mathrm{~m}(10 \mathrm{ft})$ (pink curves with circle markers) or $1.5 \mathrm{~m}$ (5 ft) (turquoise curves with diamond markers) ahead of the transmitter in the borehole. The transmitter was (a) $0.1 \mathrm{feet}$, (b) $3 \mathrm{feet}$, (c) 6 feet, (d) 9 feet, (e) 12 feet, (f) 18 feet, (g) 21 feet, (h) 24 feet, and (i) 27 feet inside the deviated borehole. 


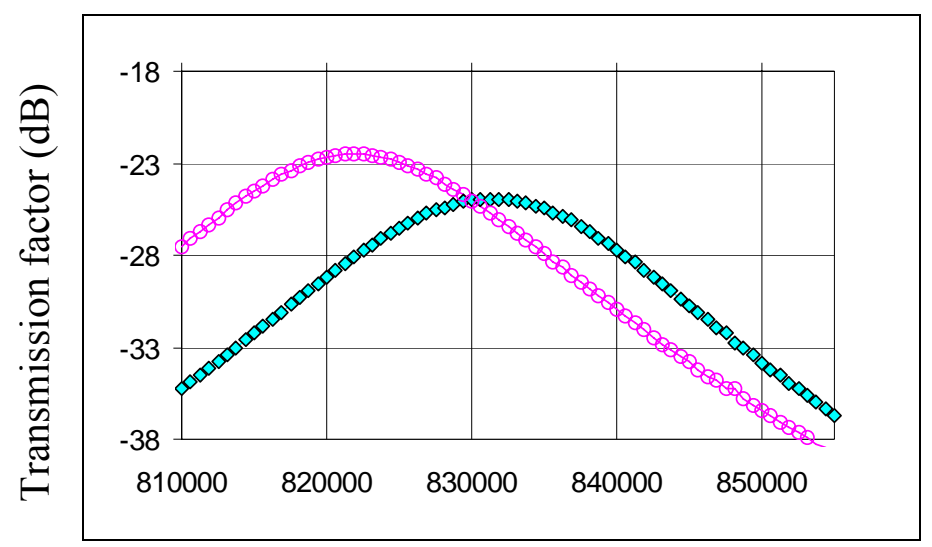

Frequency $(\mathrm{Hz})$

$(\mathrm{g})$

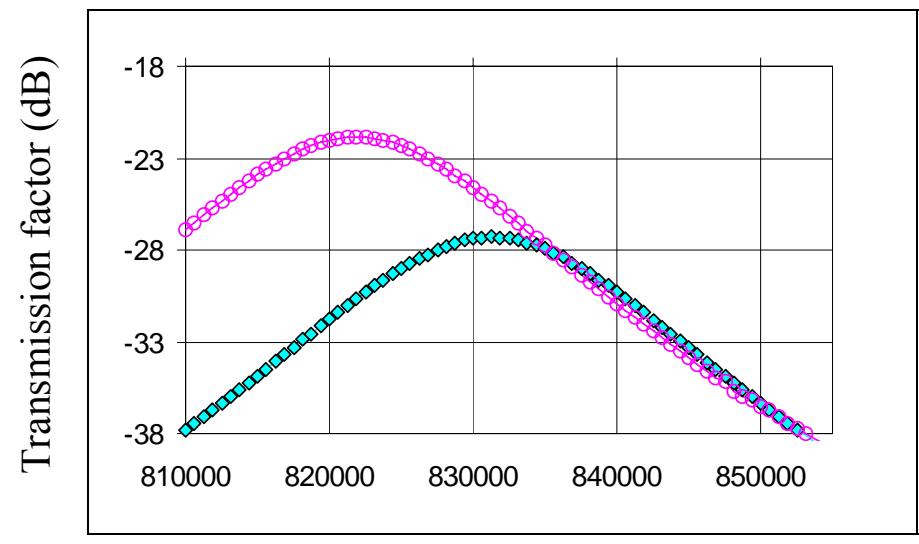

Frequency $(\mathrm{Hz})$

(h)

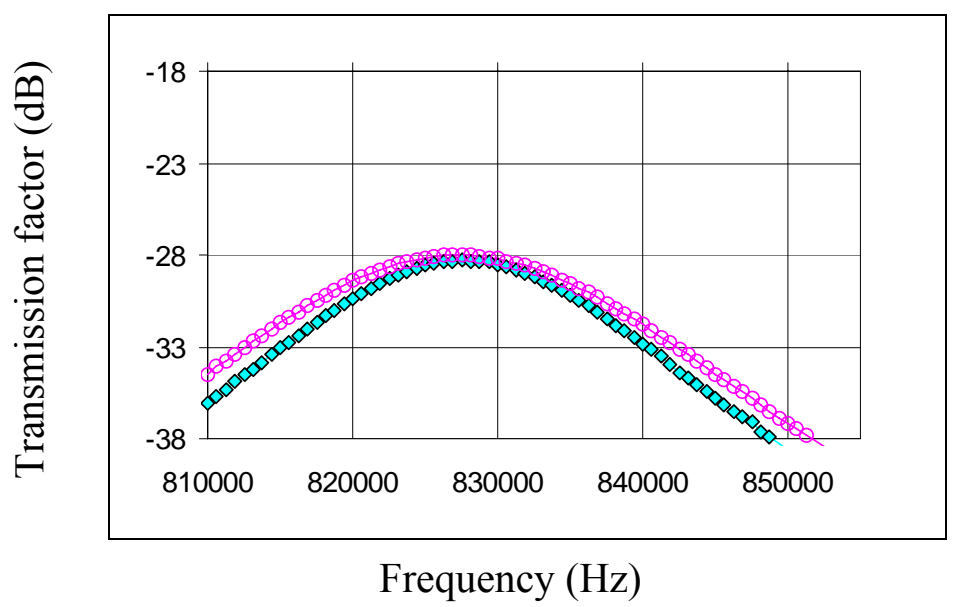

(i)

Figure 4-27. Measured transmission results with a transmitter-receiver pair of tuned loop antennas in the 15-degree deviated borehole test site. The receivers were either 10 feet (pink curves with circle markers) or 5 feet (turquoise curves with diamond markers) ahead of the transmitter in the borehole. The transmitter was (a) 0.1 feet, (b) 3 feet, (c) 6 feet, (d) 9 feet, (e) 12 feet, (f) 18 feet, (g) 21 feet, (h) 24 feet, and (i) 27 feet inside the deviated borehole. 


\subsection{Drill String Radar}

\subsubsection{Background}

To track the boundary rocks in horizontal drilling through a hydrocarbon reservoir, the type of medium surrounding the radar system, as well as the distance to the top and bottom boundary rocks, must be known. The radar system can then provide a map of the hydrocarbon reservoir, as shown in Figure 4-28, along with orientation data. To determine the type of medium being drilled, the resonant frequency of the antennas is monitored. For every radar measurement during horizontal drilling inside a hydrocarbon reservoir, the top and bottom boundary rock layers are assumed to extend indefinitely. This approximation is valid when the operating wavelengths are short compared to the extent of the object under investigation, such as in reservoirs consisting of conductive rocks. Since the coiled tubing does not rotate during the drilling process, the positions of the antennas are fairly well known at all times. With directional antenna patterns, the beam is pointed to the top and bottom boundaries separately, and the distances to the top and bottom boundary rocks are adjusted independently.

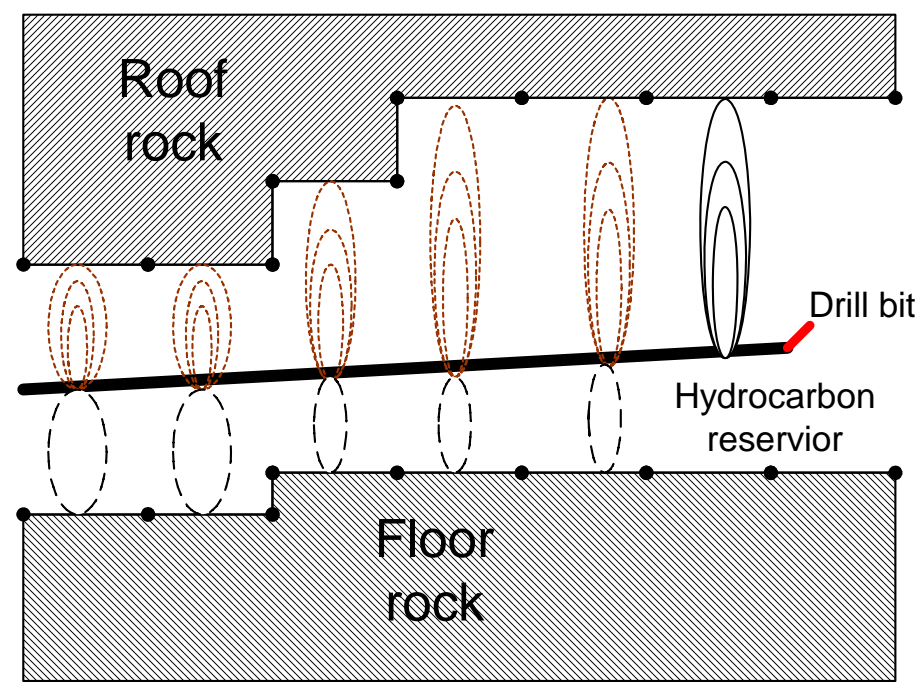

Figure 4-28. Mapping of a hydrocarbon reservoir with directional antenna patterns.

The basic Stolar Drill String Radar (DSR) technology, which is suitable for modification for applications in coiled tubing drilling environments, was initially developed with financial support through the U.S. Department of Energy (DOE) Global Initiative for the Prevention of Proliferation (GIPP) program. The DOE Kansas City Plant technical team, led by Dr. Chris Baumgart, and Stolar were the co-principal investigators on this development project. The participating MINATOM Institute for Measuring Systems Research employed 150 scientists, engineers, and technicians on this project.

A conceptual design of the DSR technology integrated into a drill string is illustrated in Figure 429. 


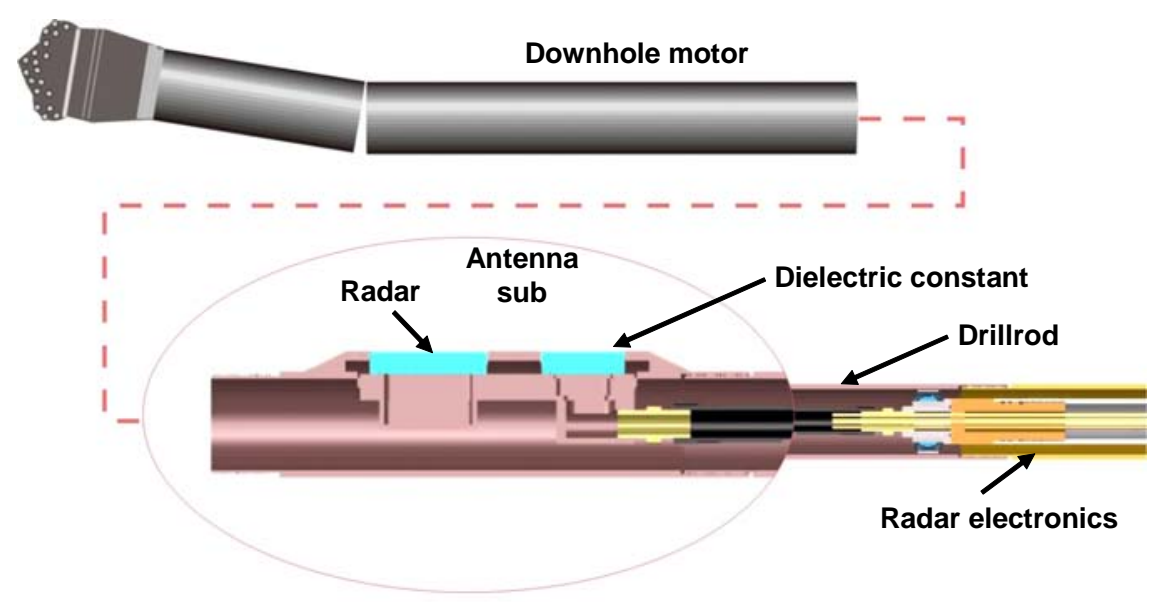

Figure 4-29. Drill string radar navigation bottom hole assembly.

The DSR has been designed with shock-mount centralizers for mounting inside a 3-inch-outsidediameter beryllium-copper drill string. The beryllium-copper drill string enables the measurement of the Earth's magnetic fields. Acceleration data also is measured for navigation and tracking of the borehole. The basic DSR design includes an inductively coupled radio data transmission system to communicate measured data to the collar and control signals back to the DSR along the drill string (see Section 2.0 and Figure 4-30). The drill string data transmission repeater(s), as necessary, is centered between the drilling rods as shown in Figure 4-30. The drill string becomes the data link to the collar transceiver and color graphics display. A separate steel drill string is the antenna subsection (see Figure 4-29).

Presently, a battery pack is used to power the DSR and data transmission system. However, a water-driven turbine generator has been developed for the DSR using DOE GIPP funding. To enable steering, a mud motor gimbal system is also under development with funds obtained through the GIPP program.

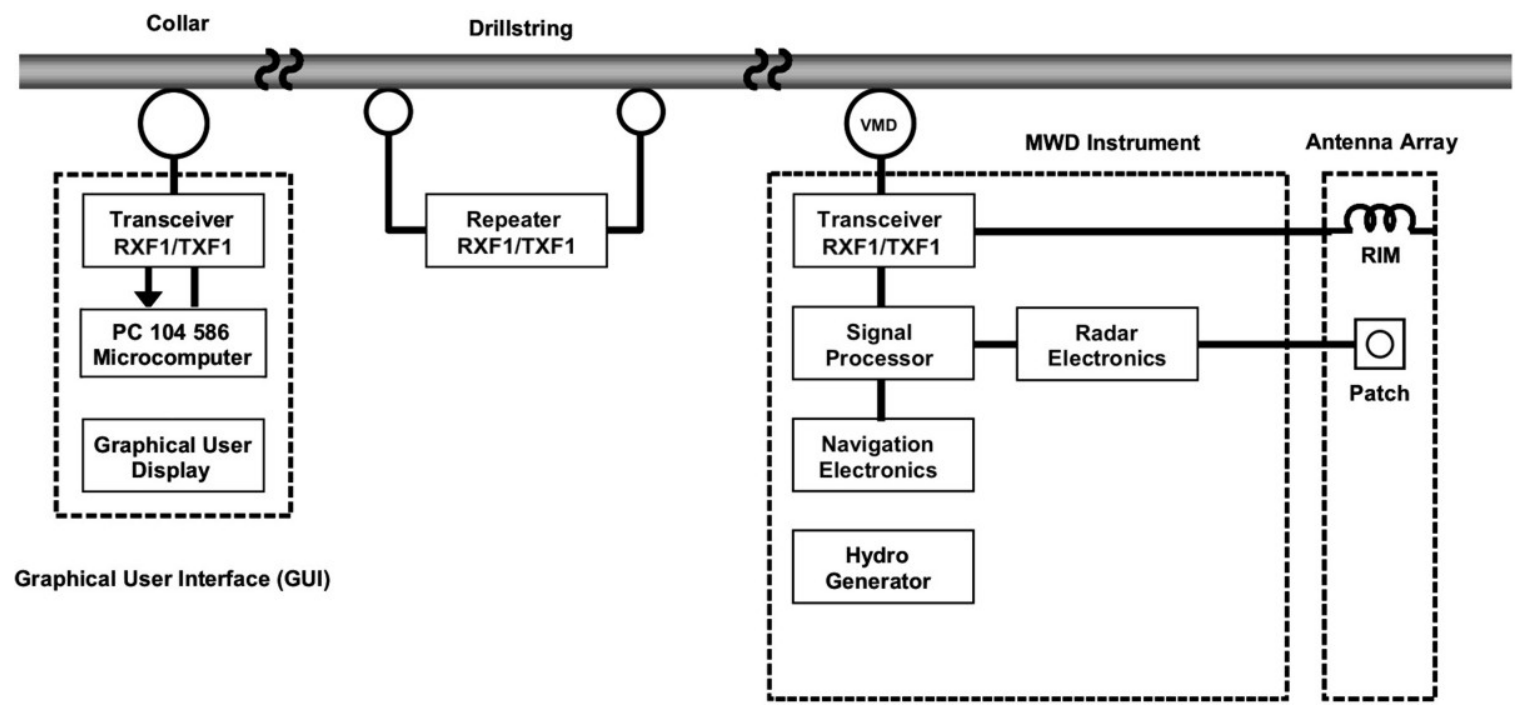

Figure 4-30. Drill string radar block diagram. 
The initial DSR technology design included a microwave patch antenna to determine to determine the in-situ dielectric constant of the media and to provide near-field detection of boundaries. A navigation sensor in the DSR is used to determine the borehole coordinates from the drill collar. The measured data are graphically displayed at the collar.

An early prototype of the DSR antenna assembly is shown in Figure 4-31. Figure 4-32 shows the DSR components, consisting of, from top to bottom in the photograph, the composite drill pipe, the transmitter and receiver radar antennas, the explosion-proof electronics enclosure, DSR electronics chassis, downhole battery pack, and downhole turbine generator.

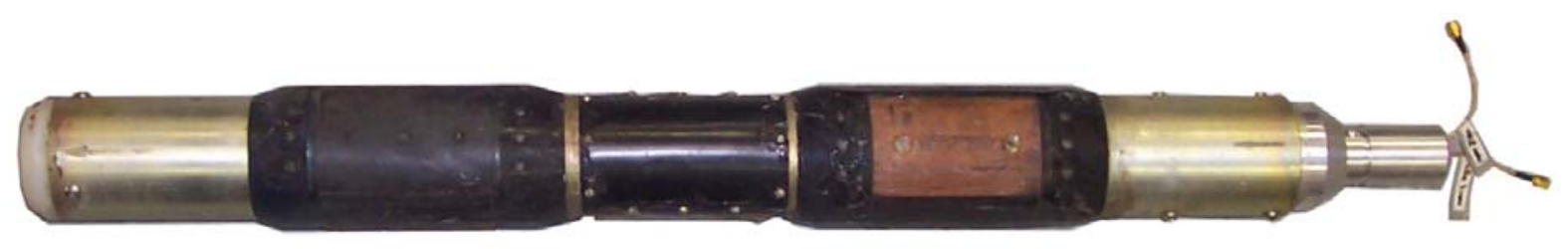

Figure 4-31. Drill string radar antennas.

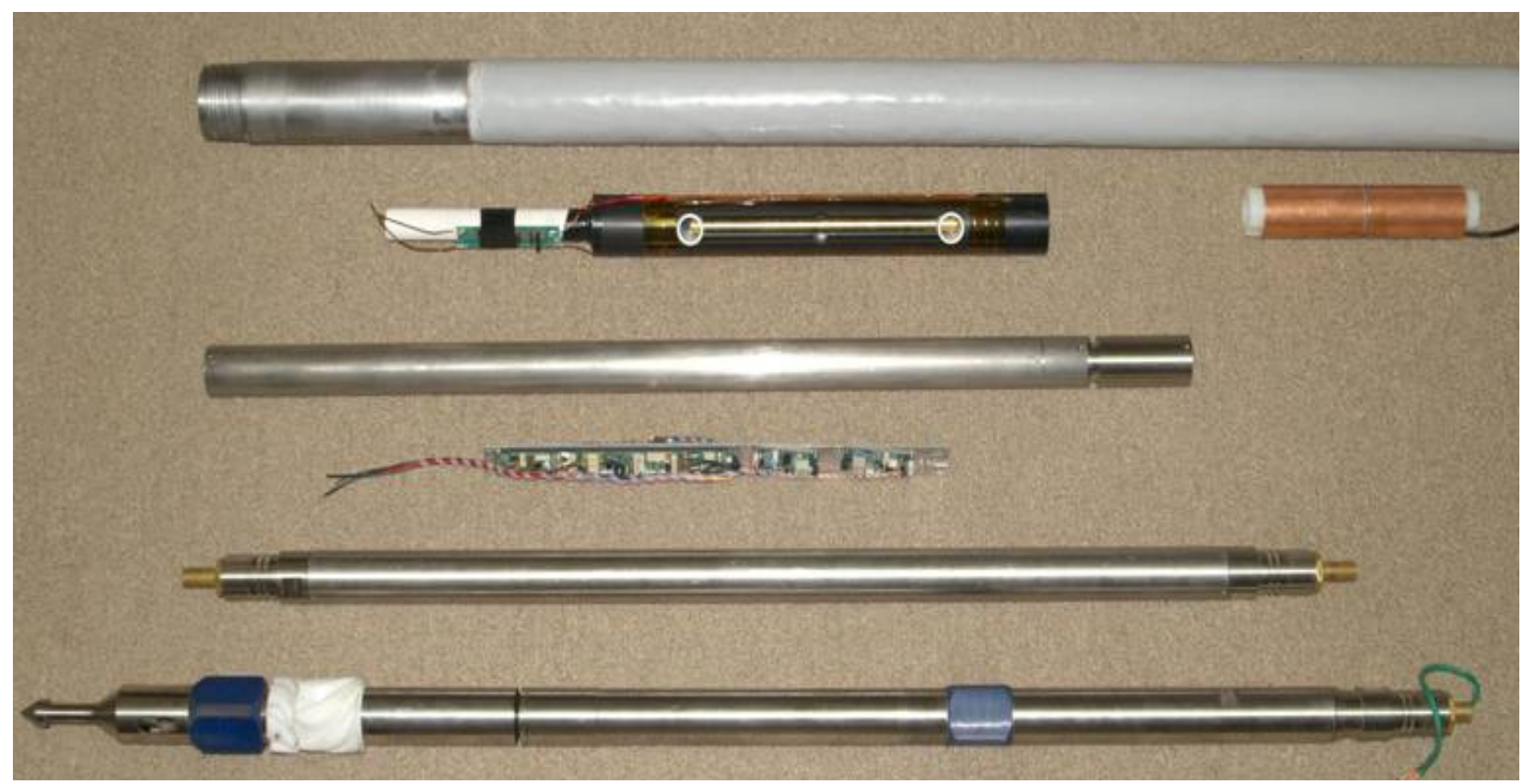

Figure 4-32. Major components of the initial drill string radar design. Shown top to bottom are the composite drill pipe, transmitter and receiver antennas, electronics enclosure, electronics chassis, downhole battery pack, and downhole turbine generator.

For some applications, pairs of step-tunable antenna elements may integrated into the basic DSR design. These antennas are separated in frequency to achieve the predistorted double-sideband, suppressed carrier waveform. A variable or constant modulation frequency radar is realized with this antenna element configuration. Tuning is achieved by switching a bank of resonating capacitors connected to the magnetic dipole of the compound antenna. The electric dipole is a 
broadband nontuned antenna. The operating frequency range extends from the low frequency to very high frequency bands. The frequencies selected depend on the petrophysics of the geologic formation.

Electrically small compound magnetic and electrical dipoles have been developed for integration into nonconductive composite cylindrical bottom hole assemblies. The compound fox hunter antenna requires phase-controlled dual transmitters. Magnitude and phase control by an SDT is required to achieve back lobe suppression of at least $15 \mathrm{~dB}$. Back lobe suppression requires approximately a $90^{\circ}$ phase shift between two coherent transmitters. The receiving antenna can be an omnidirectional sleeve dipole.

Narrow resonant microstrip patch antennas (RMPAs) have been developed for integration into the DSR technology. These antennas are mounted in shoes that are pressed with a borehole centralizer into the wall or are plowed into the wall to eliminate the drilling mud layer overlying the antenna element.

An advantage of the RMPA is that it can be applied in both near- and far-field applications. In the near field, the SDT transceiver measures the impedance. The RMPA and SDT radar are applicable in the horizontal directional drilling where stress fractures do not predominate (see Figure 4-33).

Figure 4-33. Resonant microstrip patch antenna installation.

The antennas of the initial DSR design were subjected to laboratory testing in a special test platform constructed at Stolar's laboratory (see Figure 4-34). 


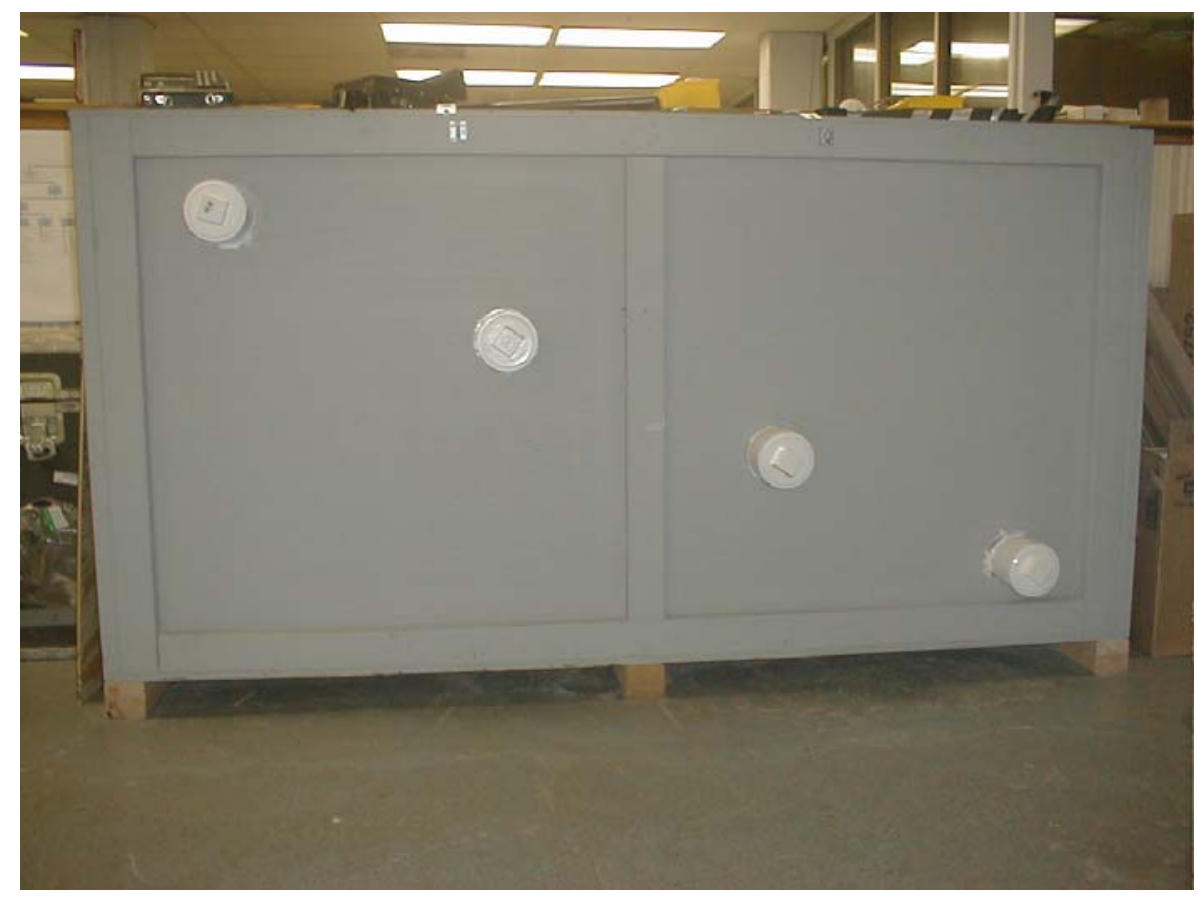

Figure 4-34. Stolar laboratory drill string radar borehole simulator.

Early proof-of-concept field-testing in a coal mining environment (see Figure 4-35) showed the need for low radio wave frequencies with separate transmitter and receiver antennas.
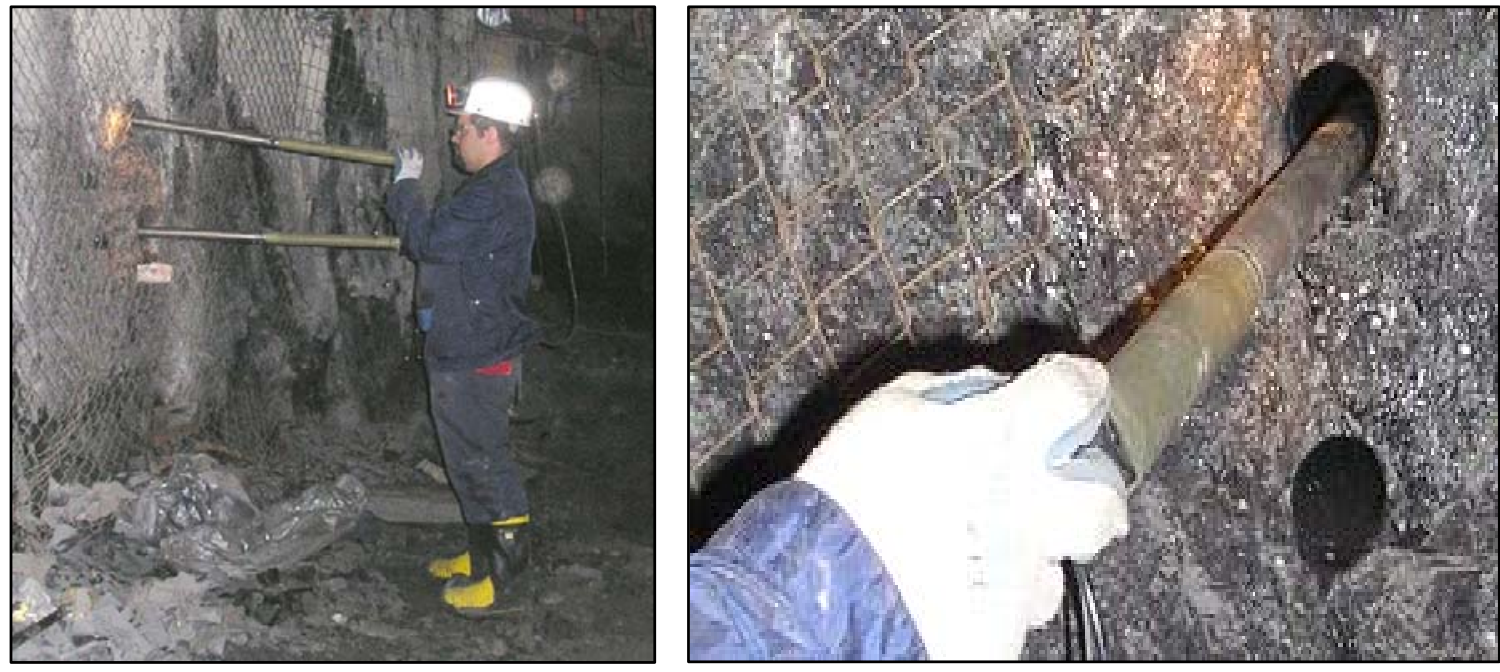

Figure 4-35. Proof-of-concept field testing of the drill string radar antennas in a coal mine.

A advanced DSR configuration employing a phased array of antennas, which allows the radar to look forward as well as sideways, is illustrated in Figure 4-36. 


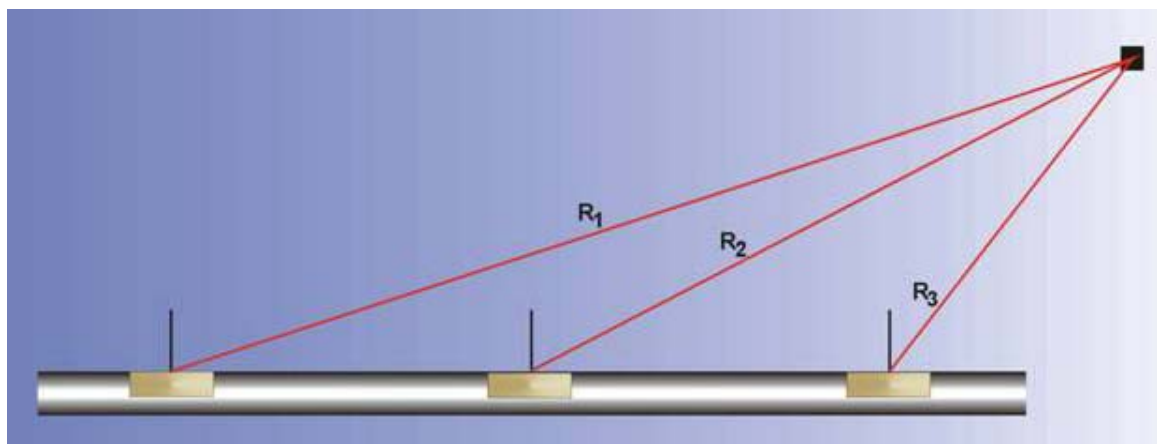

Figure 4-36. Conceptual design of a software definable transceiver drill string radar with look-ahead capability.

A phased array of antennas (e.g., patch or fox hunter) can be incorporated into the bottom hole assembly housing the DSR. Each phase is controlled to create an in-phase summary point in the natural medium, which is accomplished by using a predistorted waveform with magnitude and phase control for each antenna.

\subsubsection{Theoretical Description of the Prototype Drill String Radar Tool}

When radiating antennas are inserted into boreholes, the wave impedance $(\mathrm{z})$ is real for an electric dipole and imaginary for a magnetic dipole in the near field. The near field is defined as a distance (d) less than $\lambda / 2 \pi$, where $\lambda$ is the wavelength in meters. Energy is dissipated in a real impedance and stored in an imaginary impedance.

The implication in subsurface instrumentation design is that more energy is available to propagate into the far zone with a radiating magnetic dipole antenna when compared to a radiating electric dipole antenna. Borehole radar instrumentation requires that the transmitting and receiving antennas be collocated, resulting in crosstalk between the antennas. The magnitude of the crosstalk must be less than the magnitude of the reflected or scattered EM fields from geologic anomalies at the target distance in the far zone $(d>\lambda / 2 \pi)$ to have a sufficiently high detection signal-to-noise ratio.

A conventional, continuous-wave $(\mathrm{CW})$ radar cannot be used for detection of far-zone phenomena because this type of radar is not designed for extremely high suppression of nearzone phenomena. Detection beyond the near borehole region requires robust suppression of the near-zone EM propagation phenomena by factors exceeding $40 \mathrm{~dB}$. Greater suppression yields greater depth of investigation and near-zone suppression requires the transmission of a predistorted waveform.

Radar mapping of the near-zone has been solved by using a Green's function inversion of the measured scattered-wave data in a computerized, azimuthal tomographic algorithm. The depth of investigation has been significantly extended with predistorted waveform technology achieved by the development of a new class of radar featuring a software-definable transceiver (SDT). The SDT first operates as a conventional radar, measuring the first interface crosstalk and reflection/scattering to determine the electrical parameters of the natural media. These data enable the automatic recalibration of the radar signal path amplitude and phase shift for the 
second sequential transmission of the calibrated predistorted waveform. Subsequent reception of the scattered or reflected predistorted EM wave by the SDT orthogonal receiving antennas, enables signal processing for greater than $40 \mathrm{~dB}$ of suppression of the near wellbore EM wave phenomena. This suppression technique enables detection of targets well beyond the near zone.

Each pair of antennas is excited at the same resonant frequency. The magnetic moment (M) vectors are aligned along the $\mathrm{x}$ - and $\mathrm{y}$-axes. These magnetic dipole antennas are each driven by on-time duration, modulation amplifiers. The modulation enables the antenna pattern to be steerable in azimuth around the wellbore by forming an antenna pattern aligned along the $\mathrm{x}$-axis and then the y-axis.

The plan view of the four-quadrant antenna pattern is shown in Figure 4-37. The polarity of the front lobe electric field components is $180^{\circ}$ out of phase with respect to the back lobe. By simultaneously measuring the magnitude of the $\mathrm{x}$-axis and $\mathrm{y}$-axis receiving antenna magnetic dipoles, the direction of a geologic boundary target can be determined. The phase-coherent receiver enables the measurement of the total path phase shift between the transmit (TX) and receiver $(\mathrm{RX})$ antennas.

The radiating antenna array includes three pairs of magnetic dipoles. Each $\mathrm{x}$-axis and $\mathrm{y}$-axis dipole radiates continuous waves. A complex predistorted waveform is created by the upper antenna pair radiating at the upper frequency $\left(\mathrm{f}_{\mathrm{u}}\right)$, by the middle pair radiating at the carrier frequency $\left(\mathrm{f}_{\mathrm{c}}\right)$, and by the lower pair radiating at the lower frequency $\left(\mathrm{f}_{1}\right)$.

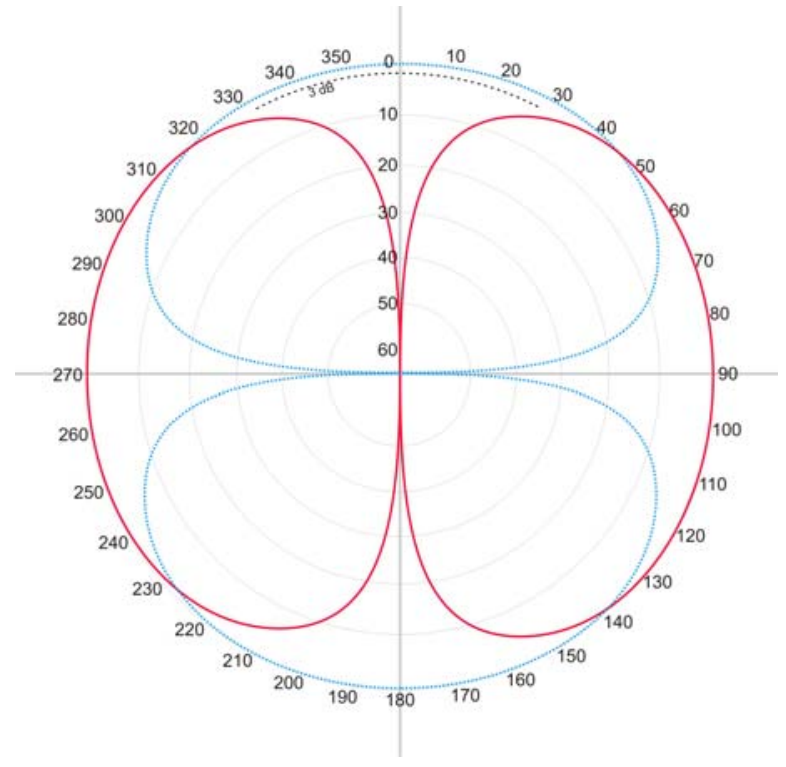

Figure 4-37. Plan view of the drill string radar prototype four-quadrant antenna pattern.

The wavelength $(\lambda)$ in meters of the propagating predistorted waveform may be approximated by

$$
\lambda=\frac{2 \pi}{\beta},
$$


with the phase constant given by

$$
\beta=\sqrt{\frac{\omega \mu \sigma}{2}} \text {; for } \frac{\sigma}{\omega \varepsilon} \gg>1 \text { radian per meter, }
$$

where $\omega=2 \pi f_{c}$,

$\sigma=$ electrical conductivity, and

$\mu=\mu_{r} \mu_{o}=$ magnetic permeability, with $\mu_{r}=$ relative magnetic permeability and $\mu_{o}=$ magnetic permeability of free space,

with $f_{c}$ given by

$$
f_{c}=\frac{4 \pi}{\lambda^{2} \mu \sigma}
$$

\subsubsection{Experimental Design of the Prototype Drill String Radar Tool}

The prototype DSR tool is shown in Figure 4-38 in a simulated test setting (see Section 4.3.4). The upper left hand side of Figure 4-38 shows the orthogonal horizontal magnetic dipole antenna array.

Figure 4-38. Prototype continuous-wave drill string radar tool in a simulated vertical test setting. 
In operation, the DSR tool first radiates a near-zone signal and the measured data are used to calibrate the signal paths. In particular, the data are used to determine the electrical parameters of the surrounding media [i.e., conductivity $(\sigma)$ if $\sigma / \omega \varepsilon>>1$ and the dielectric constant $(\varepsilon)$ if $\sigma / \omega \varepsilon<<1]$, similar to inductive logging techniques.

The second phase of operation is to set the DSR tool for suppression mode. The suppression methodology requires two or three frequency components to create the predistorted waveform, depending on the modulation type used. The SDT featured in the DSR tool design has both magnitude and phase control of each radiating frequency component, which is essential for creation of the predistortion. The heterodyne signals used in the receiver frequency mix down process also have phase control. When two frequency components are used in the design, the predistorted waveform is a double-sideband, suppressed-carrier amplitude modulated signal. Use of three frequency components results in a frequency-modulated radar.

Detection and ranging can be accomplished in two different ways using SDT technology:

- two frequency operation as a stepped-frequency, continuous-wave, (SFCW) with doublesideband, suppressed-carrier amplitude modulation (DSBSC-AM); or

- three frequency operation as a continuous-wave, narrow-band frequency modulation (NBFM).

The DSBSC-AM modulation must be used when detection and ranging of multiple scatterers or reflectors (i.e., targets) is required, which is also the mode of choice when targets are nearer to the wellbore. However, DSBSC-AM does not provide the level of near wellbore suppression that can be achieved through NBFM, which has a greater level of near borehole suppression, but the suppression range extends much further into the formation. Irregardless of the modulation type used, suppression approaches a maximum as round-trip travel time goes to zero, which essentially means that scattering and reflection from targets that are near the wellbore are eliminated from the data.

When considering the system sensitivity, the total path loss of the signal must be taken into consideration. When the loss tangent is much greater than 1 (i.e., $\frac{\sigma}{\omega \varepsilon}>>1$ ), conduction currents predominate in the natural media surrounding the wellbore. And, the predistorted waveform carrier frequency component undergoes $55 \mathrm{~dB}$ of loss and $2 \pi$ radians (i.e., $360^{\circ}$ ) of phase shift for each wavelength of travel. In addition, the spreading loss, scattering/reflection loss, and antenna mismatch loss must be added to determine the total path loss.

The first step in the practical design of an operational radar is to determine the carrier $\left(\mathrm{f}_{\mathrm{c}}\right)$ and modulation $\left(\mathrm{f}_{\mathrm{m}}\right)$ frequencies. The modulation and operating frequencies are set by the specification for the detection distance $(d)$ and the electrical conductivity $(\sigma)$ of the natural medium.

The specifications at the field test site were $d=20$ feet and $\sigma=0.05$ Siemens $/ \mathrm{m}$ or 20 ohm-m (see Section 4.3.4). Detecting a geologic boundary at a distance of 20 feet requires the total path length to be 40 feet or $12 \mathrm{~m}$ (i.e., equal to the round trip distance). Assuming that the SDT can 
achieve $40 \mathrm{~dB}$ of suppression independent of the suppression technique used, the carrier wavelength $\left(\lambda_{c}\right)$ should be

$$
\lambda_{c}=\frac{55}{40} \times 12 \mathrm{~m}=16.5 \text { meters }
$$

Thus, the carrier frequency from Equation (4-20) is given by

$$
f_{c}=\frac{4 \pi}{(16.5)^{2}\left(4 \pi \times 10^{-7}\right)\left(5 \times 10^{-2}\right)}=\frac{1}{(16.5)^{2}\left(5 \times 10^{-9}\right)}=734,618 \mathrm{~Hz}
$$

The waveform travel velocity becomes

$$
v=\lambda f_{c}=16.5 \times(734,618)=1.212 \times 10^{7} \mathrm{~m} / \mathrm{s},
$$

which implies that the waveform slows down to $4 \%$ of the speed of light $\left(3 \times 10^{8} \mathrm{~m} / \mathrm{s}\right)$. So, the round-trip travel time $(\tau)$ is

$$
\tau=\frac{12}{1.212 \times 10^{7}}=990.1 \text { nanosecond }
$$

The modulation frequency $\left(\mathrm{f}_{\mathrm{m}}\right)$ is selected to maximize the detection sensitivity at the target distance, which requires the modulation frequency to be multiplied by the waveform round-trip travel time $(\tau)$. Thus,

$$
\omega_{m} \tau=\frac{\pi}{2} \text { radian }
$$

and

$$
f_{m}=\frac{1}{4 \tau}
$$

Then, the predistorted waveform frequency components are as follows:

- $f_{c}=734,618 \mathrm{~Hz}$;

- $f_{H}=987,118 \mathrm{~Hz}$; and

- $f_{L}=482,119 \mathrm{~Hz}$.

The attenuation rate is

$$
\begin{aligned}
\alpha=\sqrt{\frac{\omega \mu \sigma}{2}} & =0.38 \text { nepers per meter } \\
& =3.3 \mathrm{~dB} / \mathrm{m} .
\end{aligned}
$$

So, the total path loss is $39.7 \mathrm{~dB}$. 
The SDT processing of the predistorted waveform results in quadrature (Q) and in-phase (I) quasistationary signals (DC) mathematically given by

$$
\begin{gathered}
\mathrm{Q}=\frac{1}{2} \cos \left(\omega_{m} \tau+\theta_{m}\right) \sin \left(\omega_{c} \tau+\theta_{c}\right) \text { and } \\
\mathrm{I}=\frac{1}{2} \cos \left(\omega_{m} \tau+\theta_{m}\right) \cos \left(\omega_{c} \tau+\theta_{c}\right) .
\end{gathered}
$$

The heterodyne signal phase $\left(\theta_{\mathrm{m}}\right)$ is set by the microprocessor following the measurement of near-mode calibration data. The carrier signal phase $\left(\theta_{c}\right)$ also can be set by the microprocessor. Just before the far-mode measurement is made, the heterodyne phase is set such that $\theta_{\mathrm{m}}=\pi / 4$.

The carrier phase is then given by

$$
\omega_{c} \tau+\theta_{c}=\tan ^{-1}\left(\frac{\theta}{I}\right)
$$

and the magnitude

$$
\begin{gathered}
A=\sqrt{Q^{2}+I^{2}} \\
=\cos \left(\omega_{m} \tau+\theta_{m}\right) .
\end{gathered}
$$

After calibration, the carrier phase $\left(\theta_{\mathrm{c}}\right)$ is set to zero by the microprocessor just before the farmode predistorted transmission.

The total path delay is then

$$
\tau=\frac{\tan ^{-1}\left(\frac{Q}{I}\right)}{\omega_{c}}
$$

and the distance $(\mathrm{d})$ is determined from

$$
\mathrm{d}=v \times \tau,
$$

where the velocity is determined from the near borehole measurement of electrical conductivity.

The direction of a geologic boundary can be determined by radiating sequentially from the $\mathrm{x}$-axis and then the $y$-axis magnetic dipole. Measurement of magnitude $(A)$ and phase $\left(\theta_{c}\right)$ determines the direction from the wellbore.

The determination of distance by measuring phase will exhibit an ambiguity of \pm 2 electrical degrees. With a phase constant $(\beta)$ of $17.9^{\circ} / \mathrm{m}$, the range ambiguity is 


$$
\Delta \mathrm{d} \approx \pm 0.11 \mathrm{~m}( \pm 4.3 \mathrm{in} .)
$$

The round-trip travel time $(\tau)$ depends on the velocity $(v)$ of the EM wave in the medium and the path distance. Figure 4-37 indicates the $180^{\circ}$ phase difference between the front and back lobes of the radiating horizontal magnetic dipole.

The phase shift of the predistorted waveform exhibits a carrier phase shift $\left(\theta_{c}\right)$

$$
\theta_{\mathrm{c}}=\omega_{\mathrm{c}} \pi \text { radians }
$$

and a modulation phase shift

$$
\theta_{\mathrm{m}}=\omega_{\mathrm{m}} \mathrm{T}
$$

The scattered and reflected signals arriving from the targets are each delayed in time $(\tau)$, resulting in the modulation and carrier phase shifts. Each received (RX) signal is processed by the SDT, producing in-phase (I) and quadrature (Q) signals that can be represented by a phaser on polar plot of concentric suppression rings (see Figure 4-39).

In Figure 4-39, the red curve represents the modulation near-zone suppression factor. The nearzone suppression reaches a maximum value for small values of $\omega_{\mathrm{m}} \tau$ and a minimum value for values of $\omega_{\mathrm{m}} \tau \rightarrow \pi / 4$. As illustrated in Figure 4-39, each received scattered or reflected EM wave is represented as a phaser with phase shift, $\omega_{\mathrm{m}} \tau$, referenced from $\theta=0$,. In the near zone represented by the blue curve, the small modulation phases angle phasers are not suppressed. In the far zone, represented by the red curve, the small modulation phase angle phases are significantly suppressed, while far signal suppression vanishes when $\omega_{\mathrm{m}} \tau$ increases above $70^{\circ}$.

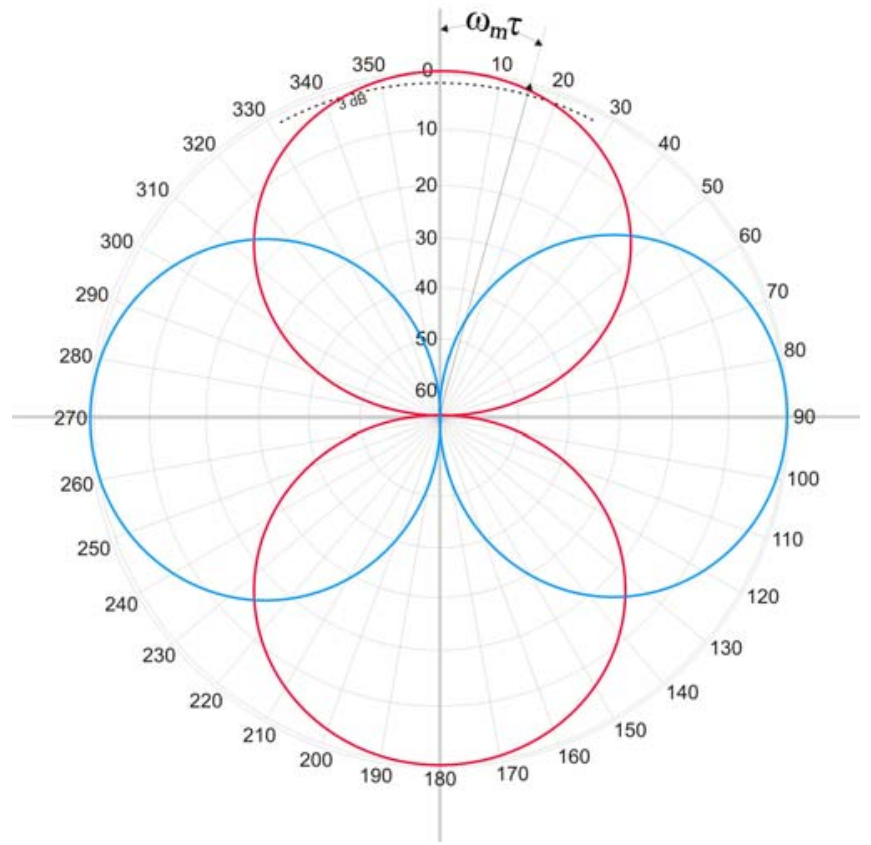

Figure 4-39. Polar suppression factor represented by near- (red) and far- (blue) zone suppression superimposed on concentric circles. 


\subsubsection{Experimental Results for the Prototype Drill String Radar Tool}

\subsubsection{Field Test Description}

Initial field tests of the prototype DSR tool where conducted over a three-day period at the test site illustrated in Figure 4-40.

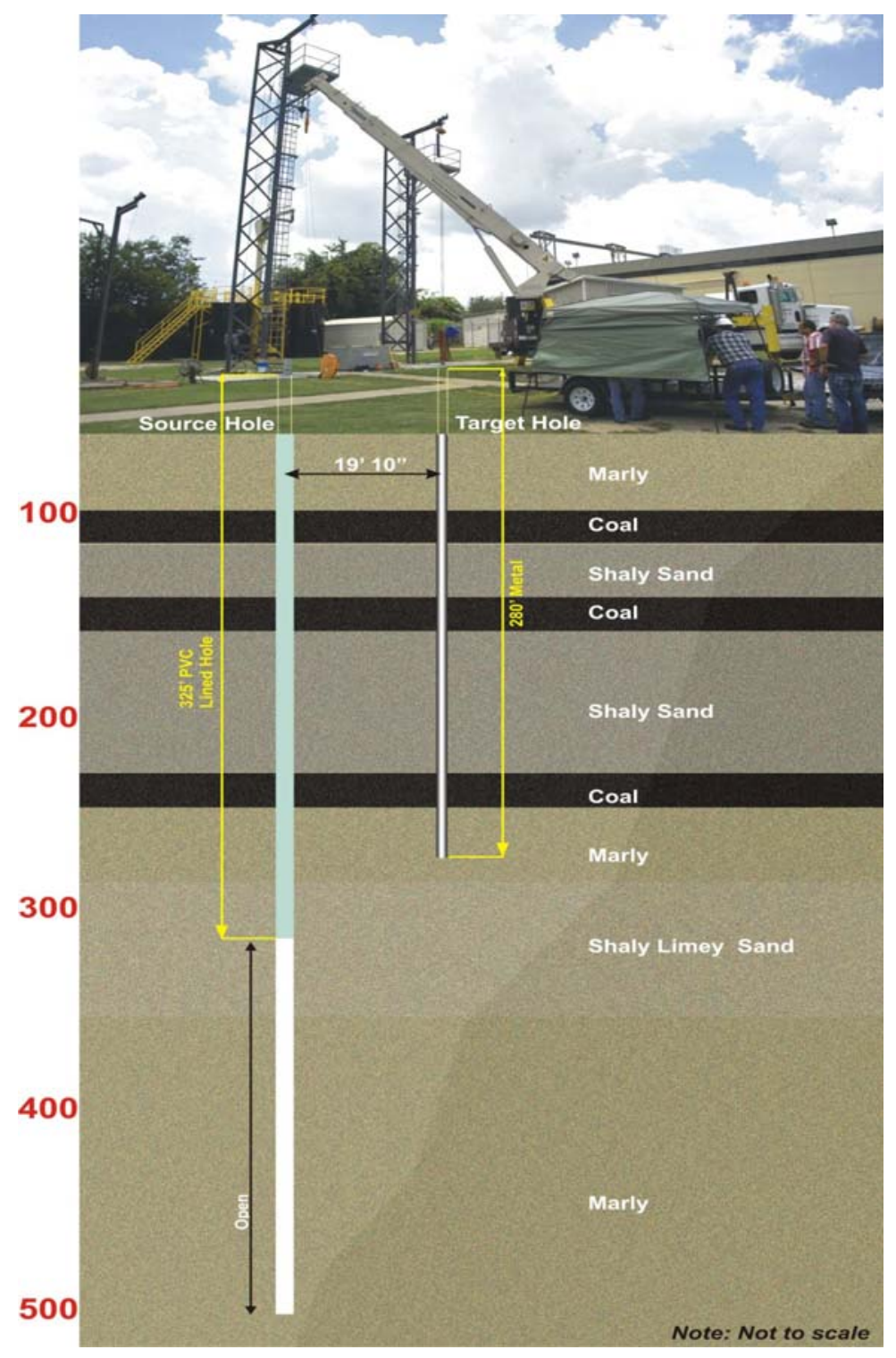

Figure 4-40. Field test site and subsurface geology.

The major objectives of this test were to:

- demonstrate the field deployability of the prototype DSR tool; and

- demonstrate the ability of the prototype DSR tool to "target" a metal cased borehole, simulating a geologic boundary, within a radius of 20 feet from the borehole containing the prototype DSR tool. 
As shown in Figure 4-40, the test site consisted of two boreholes with supporting structures to help place tools in and out of the boreholes. At the surface, the two boreholes are spaced 19 feet 10 inches center-to-center, but because the two boreholes have not been surveyed to depth, the deviations away from the surface separation distance are not known. When viewing the test site face on, as shown in Figure 4-40, the left borehole was used as the borehole for emplacing the prototype DSR tool and the steel casing in right borehole was the target. The former hole has a total depth of 500 feet and is PVC cased down to a 375-foot depth. The remainder of the borehole from a depth of 375 feet to total depth (TD) is open hole. The right borehole, the target borehole, is 280 feet in total depth and is lined with metal casing from the surface to TD.

The test plan included placing the prototype DSR tool into the borehole as shown in Figure 4-41. The prototype DSR tool operates as a transceiver with separated receive and transmit sections (see Figure 4-41). Power for the transmit section, data, and command and control from the surface unit to the prototype DSR tool are all carried along a 7-conductor wireline.

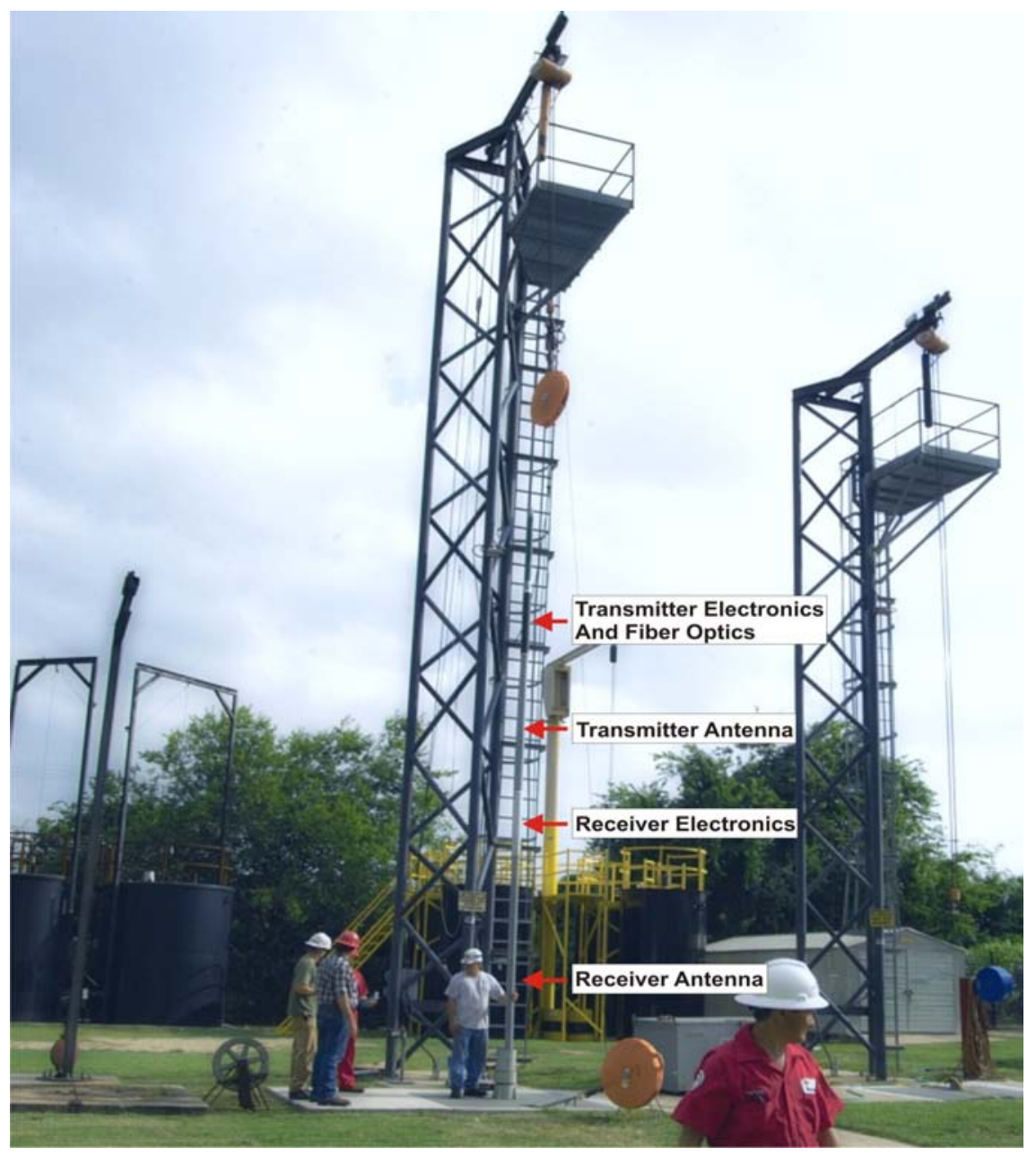

Figure 4-41. Prototype drill string radar tool prior to being lowered into the source borehole. The four major sections of the tool assembly are indicated. 
From the surface, the prototype DSR tool was controlled via a laptop computer, running proprietary software (see Figure 4-42). The software is portable and may be integrated into any platform running Windows OS. At this stage, software control of the prototype DSR tool was not automated and required expertise to properly operate the system. Figure 4-43 shows a computer screen shot of part of the interface needed to control the prototype DSR tool.

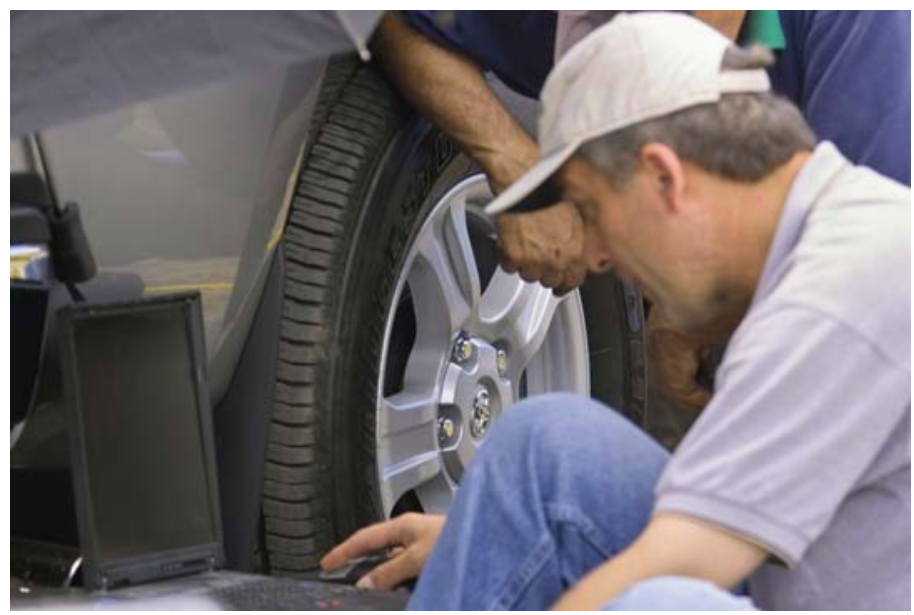

Figure 4-42. Command and control of the prototype drill string radar tool being executed via a laptop computer.

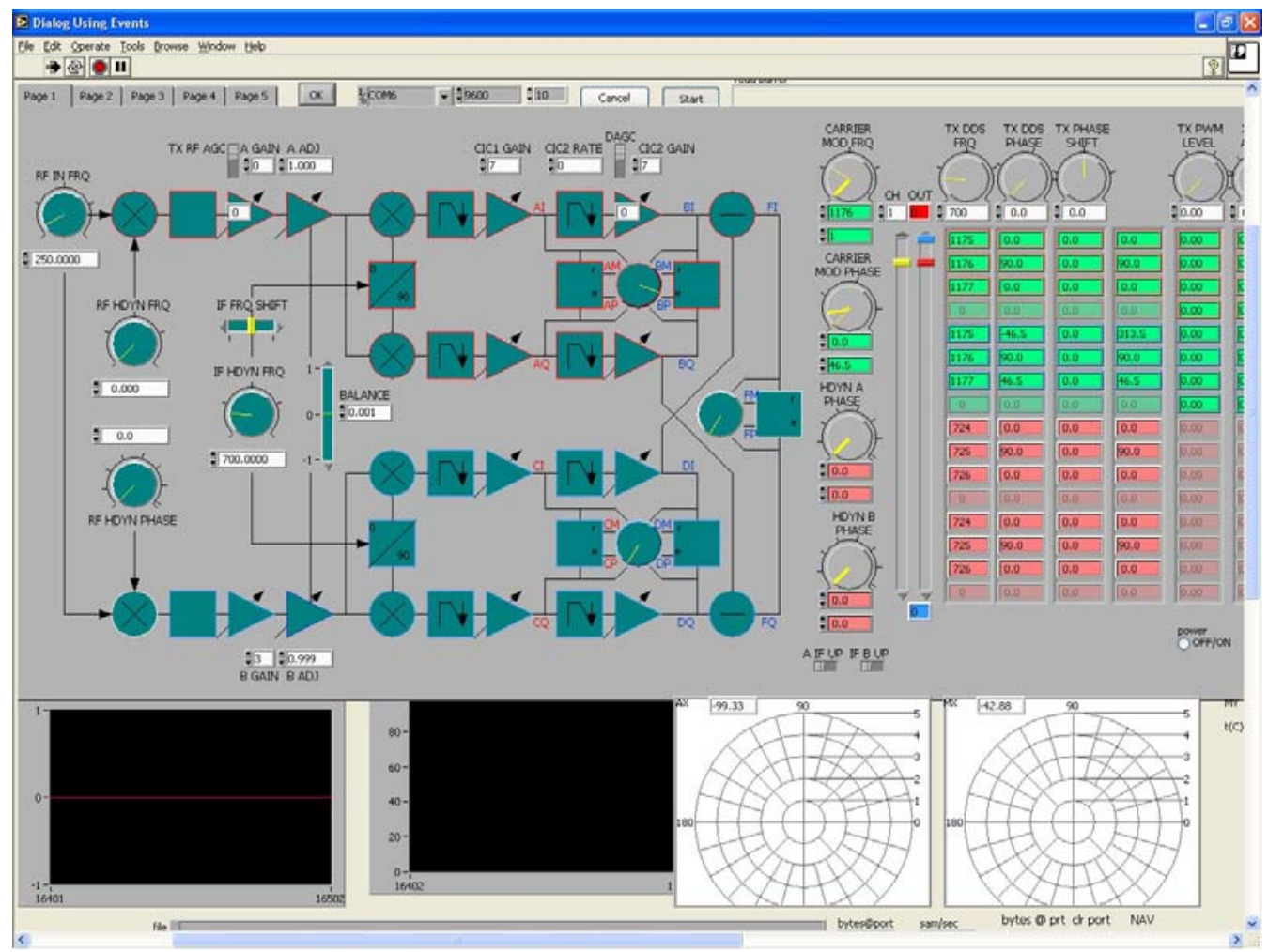

Figure 4-43. Computer screen shot of the surface graphical user interface for command and control of the prototype drill string radar tool. 
Control of the prototype DSR tool requires both the transmitter (TX) and receiver (RX) sections to be commanded individually. Further, each section must be tuned separately. The graphical user interface shown in Figure 4-43 is only one of five pages that must be "tweaked" in real time to set up the system for proper operation. This procedure is not routine and requires an operator with extensive knowledge of the theory of operation for the prototype DSR tool. Some of these limitations on operator proficiency are due in part to the nature of prototype testing. Access to and understanding of the actual operation of the prototype DSR tool in situ will help form the understanding necessary way for autonomous operation of the DSR tool in future development cycles.

Both transmitter and receiver sections of the prototype DSR tool must be calibrated separately and then jointly for proper system operation. The prototype DSR tool has capability to generate three operating frequencies simultaneously. The number of frequencies generated is a result of the type of "radar" or mode in which the system is operating. On the transmit side, there are two tuned antennas per frequency, forming an orthogonal horizontal magnetic dipole (HMD) antenna pair. In all, there are six antennas, making up three orthogonal pairs, on the transmit side. On the receiver side, only a single, wideband, orthogonal antenna pair is used. So, in all, there are eight antennas that need to be calibrated, which is a nontrivial, manual task. Future design efforts will fully automate this process.

During the calibration process, the magnitude and phase for each transmitter must be accurately set, in part, to offset inherent receiver antenna pattern influences that could cause artifacts in the measured data. In addition, the near-field (i.e., direct) coupling of the transmitters into the receiver antennas needs to be determined in order to more precisely suppress the near-field components of the radar return.

In operation, data are collected at $11.25^{\circ}$ increments around the borehole. The beam steering is a result of amplitude and phase variations in the transmitter antennas. Because the data collection process is not automated, the amplitude and phase of the transmit antennas must be set for every data point through the control interface on the surface computer, depending on whether or not the system is looking in the near field, or in the near-field suppression (i.e., far-field), mode. The exception to this requirement is when the prototype DSR tool was being used in an inductive logging mode. In this case, since data from only a single frequency were being mapped, continuous adjustment of the transmit antennas was not required.

Regardless of the mode of operation, data collected by the receiver were sent in raw form up the wireline to the surface computer for post processing. Minimal processing was conducted down hole. The raw data had transmitter configuration and navigation pack data appended before being sent up hole. Figure 4-44 shows a screen shot of one page of post-processed data on the surface computer. The data represented in Figure 4-44 are a short time sample of a streaming inductive logging run that the prototype DSR tool performed while being withdrawn from the borehole. The data shows changes as the prototype DSR tool transitioned between the depths of 290 and 270 feet, which were in good agreement with the logs provided for the source borehole. Though interesting and certainly indicative of a properly operating system, no other analysis on this particular data set was conducted. 


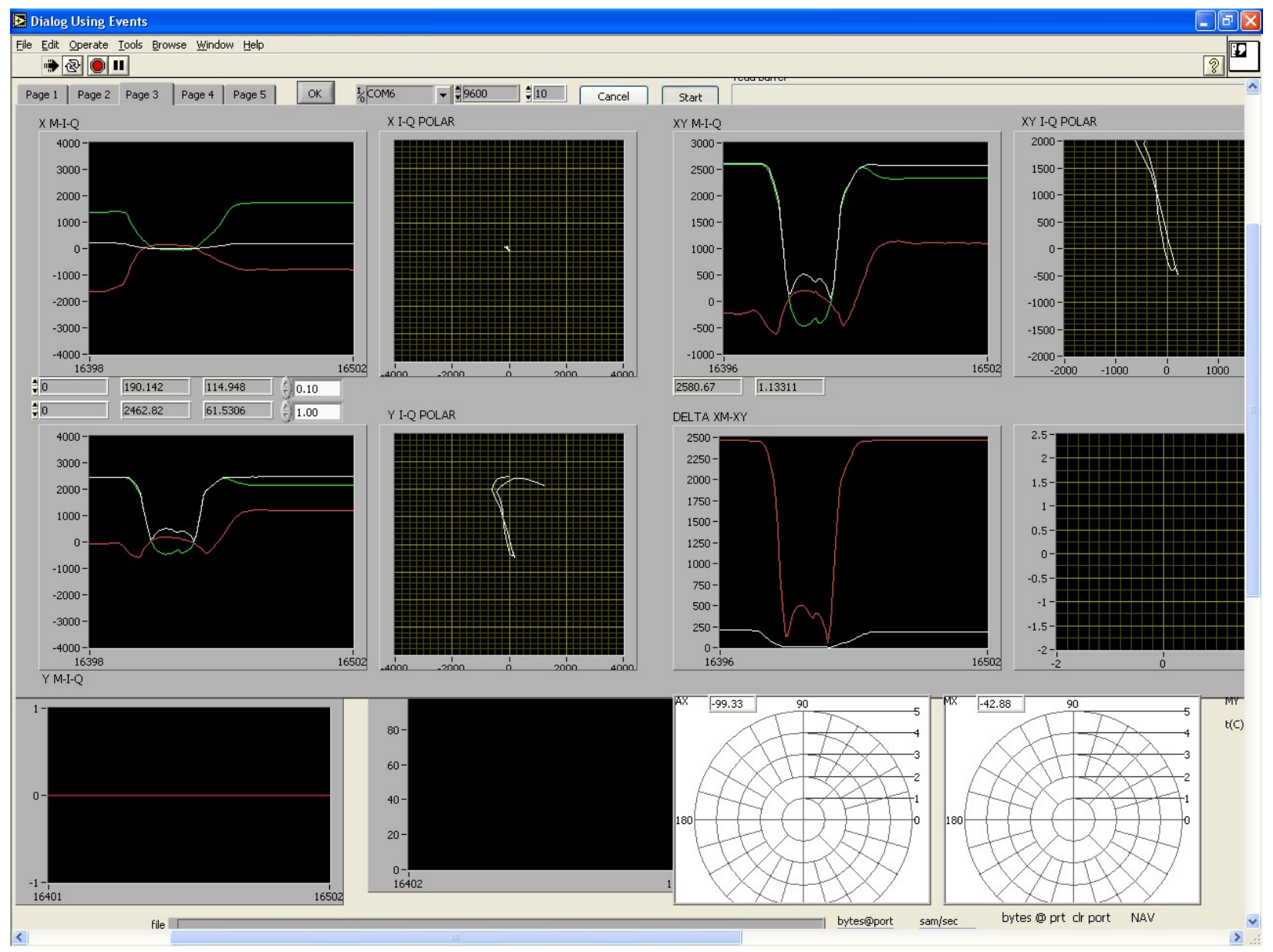

Figure 4-44. Computer screen shot of one page of raw and processed data.

The original testing plan called for calibration and target locating at various depths with varying resistivities. However, several intermittent system failures during the course of the early testing routines proved to be too problematic for collecting any meaningful data through the first day and one half of testing. The failures were due to fabrication errors in the prototype system, primarily as a result of a failed cable head connector. Delays in testing were also exacerbated by software modifications that were needed before the prototype DSR tool could be placed downhole. In all, a day and one half of field time was spent trying to fix the prototype DSR tool and upgrading the control software. As a result, the quantity of data that was actually collected over the course of the entire test was far less than expected.

The prototype DSR tool and the control software were deemed operationally sound in the late afternoon of the second day of testing. After that determination, with all system modifications and failure corrections in place, the prototype DSR tool performed flawlessly for the remainder of the test. The afternoon tests during the second day were primarily focused on setting the parameters of the prototype DSR tool, tuning and calibration, operation in near field mode, and testing the operation of the navigation pack module. The morning of the last day of testing focused primarily on doing measurement repeatability testing of the prototype DSR tool and on one run of calibration and target location. 


\subsubsection{Field Testing Procedures}

The antenna array design in the prototype DSR tool enabled various types of functionalities to be evaluated. The functionalities were evaluated by activating combinations of antenna configurations with the transmitter-receiver (TX-RX) array illustrated in Figure 4-45. The transmitter and receiver amplifiers have associated programmable direct digital synthesizers that are frequency, magnitude (i.e., gain), and phase programmable.

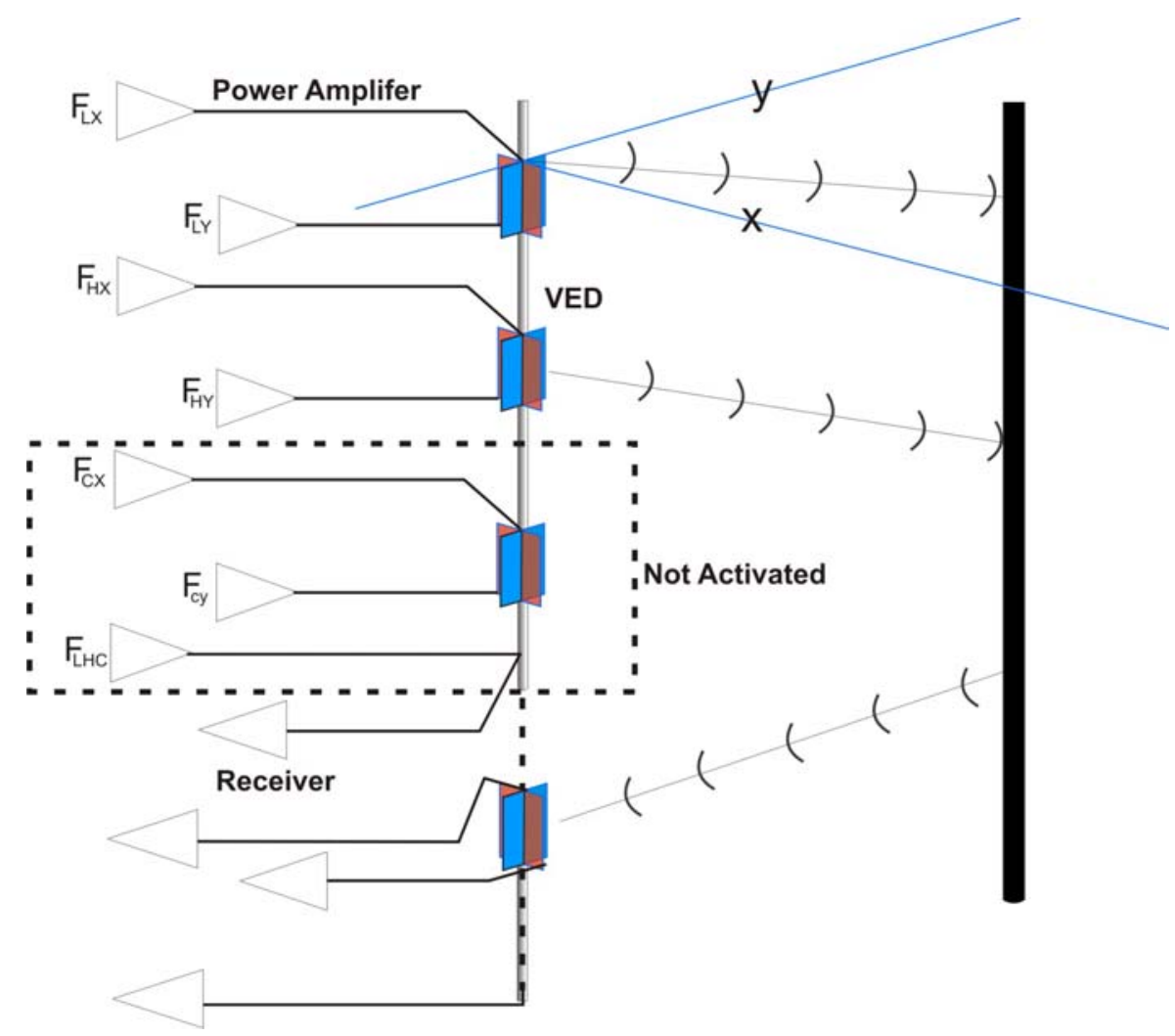

Figure 4-45. Collocated quadrature transmitting and receiving antenna array in the prototype drill string radar tool.

The combination of orthogonal transmitting antennas forms a predistorted transmit waveform. The predistorted waveforms are either double-sideband, suppressed-carrier or a special case of frequency modulation. Given the current stage of development of the prototype DSR tool, calibration was accomplished by control functions set up in Labview software (see, for example, Figure 4-43). The calibration function will be automated when the operating radar antenna configuration is selected during further planned field testing.

\section{Transmitting Array Calibration}

The orthogonal transmitting antenna array is configured with three pairs of resonant horizontal magnetic dipoles (HMD) phase coherently driven by direct digital synthesizers and highefficiency, rectangular, on-time duration, modulated power amplifiers (Class-L). The on-time 
duration modulator controls the magnitude of each radiating HMD. The transmitting array includes a vertical electric dipole (VED) antenna, which is connected to a receiver during the transmitting array calibration procedure.

During calibration, the on-time modulation $\mathrm{F}_{\mathrm{LX}}$ and $\mathrm{F}_{\mathrm{LY}}$ amplifiers are turned on and are received simultaneously by the wideband omnidirectional VED antenna. Each pair of HMD transmitting antennas is calibrated to generate a rotating constant magnitude vertical electric field pattern electronically steered around the wellbore. The phi $(\phi)$ azimuth angle designates the direction of the maximum of the figure-eight antenna pattern. The front lobe electric field component is $180^{\circ}$ out of phase with the back lobe.

The transmitting array may utilize the calibrating antenna to form a "fox hunter" VED for backlobe suppression. This functionality was not activated during the testing program.

\section{Receiving Antenna Calibration}

A pair of orthogonal non-resonant HMD antennas and a single VED formed the receiving array. The VED may be used as a "fox hunter" receiving antenna to suppress the back lobe. The VED may also be used for electric field suppression. These functionalities were not activated during the testing program due to limitations in the receiver electronics design.

\subsubsection{Analysis of the Collected Data}

The operational problems encountered with the DSR prototype tool were severe and required most of the available time to resolve. As a consequence, only a small percentage of the functionalities of the DSR prototype tool were demonstrated.

A polar plot of the acquired data is illustrated in Figure 4-46.

\section{Transmit $F_{L X}$ and $F_{L Y}$ Calibration with the Built-In Vertical Electric Dipole Antenna Receiver}

First, the orthogonal pair of transmit antennas were calibrated to form a phi $(\phi)$ angle rotation with a constant magnitude electric field component. The phi $(\phi)$ sweep illumination of the steel casing target in the companion wellbore generated a conduction current in the steel casing and a scattered wave detection in the receiving coil. These two electromotive forces are depicted as voltages in the array $x$-axis (blue trace in Figure 4-46) and y-axis (green trace in Figure 4-46) HMDs.

The steel casing was observable at the phi $(\phi)$ rotation angle of $102^{\circ}$ (see Figure 4-46). The cross talk was successfully suppressed to a very small value as is indicated by the plotted values near the origin of the polar graph in Figure 4-46.

Receiver $F_{L X}$ and $F_{L Y}$ Response

The phi $(\phi)$ azimuth angle sweep for the transmitting antenna array pattern as measured in the receiving antenna array detected the steel casing. The detection signal-to-noise ratio was very 
high. The noise value includes cross talk and clutter from the near-borehole scatter. The high near-borehole suppression was obtained via digital processing in the software definable transceiver.

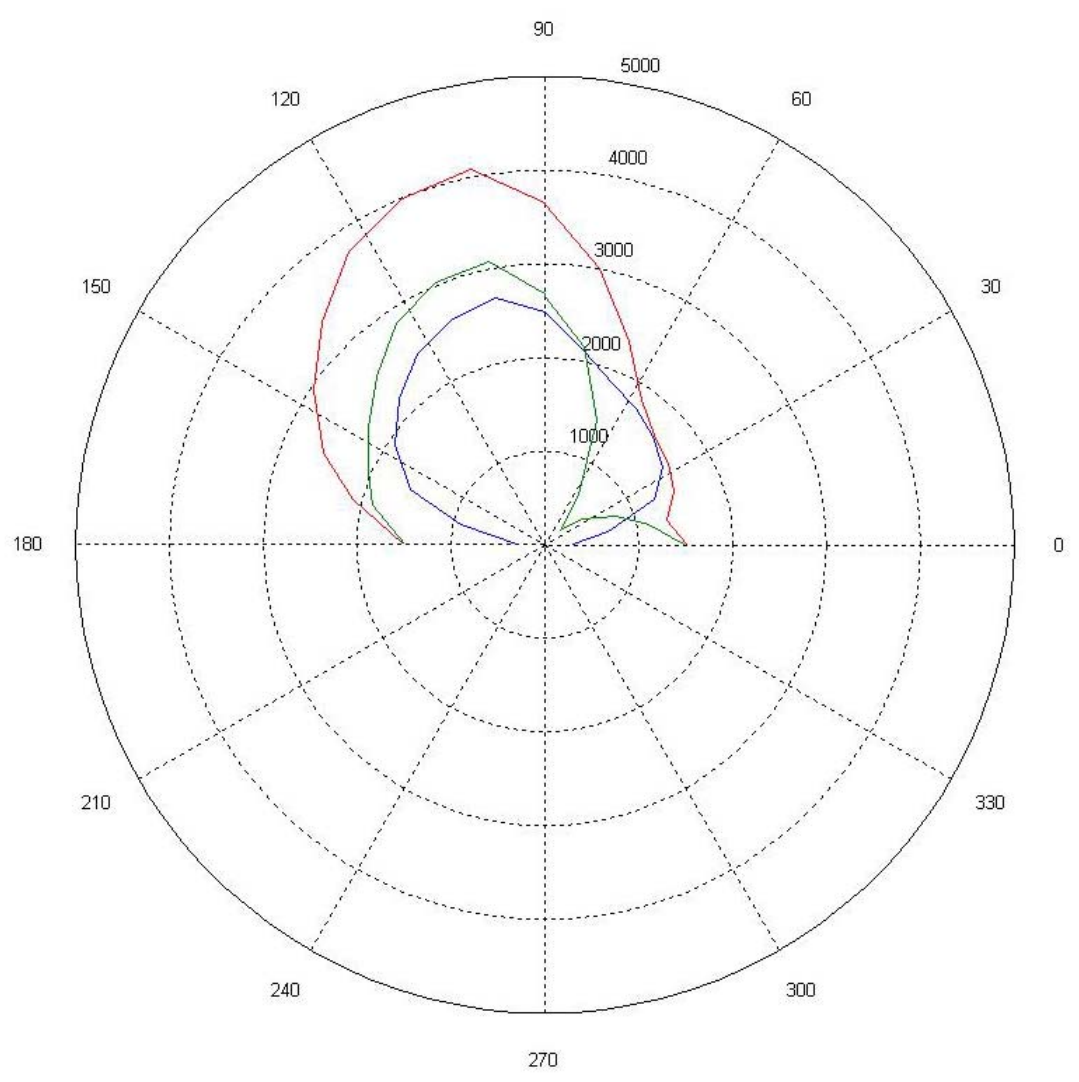

Figure 4-46. Graphical presentation of the acquired receiver data. The receiver $x$ and $y$ directed HMD coil voltages (blue and green traces, respectively) and the magnitude of the combined coil voltages (red trace) are shown.

The phi $(\phi)$ azimuthal sweep of the orthogonal transmitting HMD array detected the target in the second quadrant. However, because of the erroneous data provided by the navigation subsystem, the actual orientation (i.e., bearing) of the target could not be determined.

The determination of the range to the target requires the measurement of the total path phase shift. In general, the electrical phase shift can be measured with respect to the calibrated predistorted waveform suppressed carrier frequency. The apparent initiating phase center can be determined during calibration of the transmitting array. The shift in phase to the receiving array determines the roundtrip distance. Time constraints during the testing did not permit working out the fine details of the distance to the target measurement procedures.

\section{Measurement Repeatability}

Two data collection repeatability tests for the prototype DSR tool were conducted on the final day of testing. Time constraints only allowed for two different depths to be investigated for data 
collection consistency (i.e., 230 feet and 180 feet end of tool depth for a separation of 50 feet). First, a series of measurements were taken at 230 feet. Then, the DSR tool was raised to 180 feet and a second series of measurements were made. The DSR tool was then lowered back to a depth of 230 feet and the process was repeated. These tests were conducted with the DSR tool operating in a dual frequency mode and the transmit antenna patterns rotating from 0 to $90^{\circ}$.

Unfortunately, the saved data file for the second set of measurements at 230 feet was corrupted. This failure was not discovered until post processing of the data was performed at Stolar's facilities in Raton so only the measurements at 180 feet can be compared. The data for the two sets of measurements taken at 180 feet are shown in Figure 4-47.

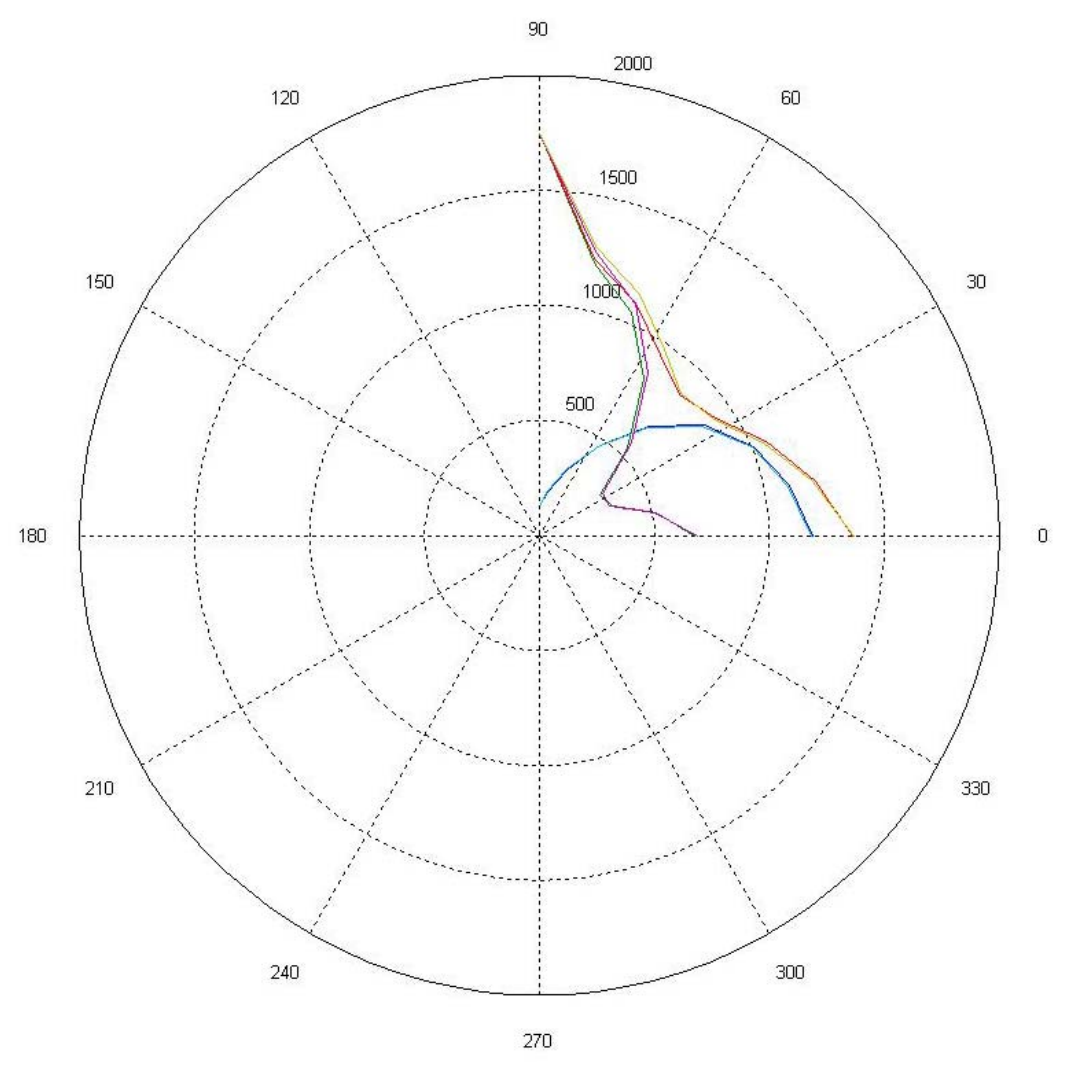

Figure 4-47. Data for the two separate sets of measurements taken at a depth of 180 feet.

The data plotted in Figure 4-47 represent the magnitude of the receive signal at each of the two receiver antennas (i.e., receiver orthogonal antenna pair), with a third set of data showing the combined magnitude of the receive signal. The cyan (x-axis antenna) and green (y-axis antenna) traces are data from the first set of measurements at 180 feet, with the yellow trace corresponding to the magnitude of the total field at the receiver $\left(\sqrt{x^{2}+y^{2}}\right)$. Likewise, the blue ( $\mathrm{x}$-axis antenna) and purple (y-axis antenna) traces are data from the second set of measurements at 180 feet, with the red trace being the total field magnitude data.

Comparison of the traces representing the two sets of measurements taken at 180 feet (see Figure 4-47) indicates very good consistency and repeatability for data collection by the prototype DSR 
tool. However, Figure 4-47 only addresses measurement repeatability and does not infer any information with respect to the target distance or bearing (azimuth).

\subsection{Coal-Based Applications of the Prototype Drill String Radar Tool}

The Stolar DSR tool has important applications in coal bed methane (CBM) and coal mine methane (CMM) extraction. In CBM production, the ability to accurately guide horizontal drilling in thin coal seams holds out the potential to dramatically reduce the requirements for dewatering the seam prior to initiating methane production. This capability will, thus, substantially mitigate the surface environmental impact of produced water. Moreover, accurate horizontal drilling within a coal seam will avoid introducing flow permeability restrictions by intersecting mudstone, fire clay, or sedimentary rock boundary layers, which results in these materials being injected into the near borehole pore space. More accurate horizontal drilling will increase overall CMM drainage rates.

Because of differential compaction in coal deposits, undulations in coal seams occur naturally (see Figure 4-48). The undulating nature of coal seams, especially under meandering paleochannels, causes directional drilling problems often requiring sidetracks (see Figure 4-49). Sidetracks, which can decrease the drilling efficiency substantially, are commonly used in a trialand-error drilling method to maintain the borehole within the undulating coal bed by identifying the vertical limits of a layered formation by repeatedly drilling into the top and bottom of the formation. Use of the prototype DSR tool would eliminate this inefficient drilling technique.

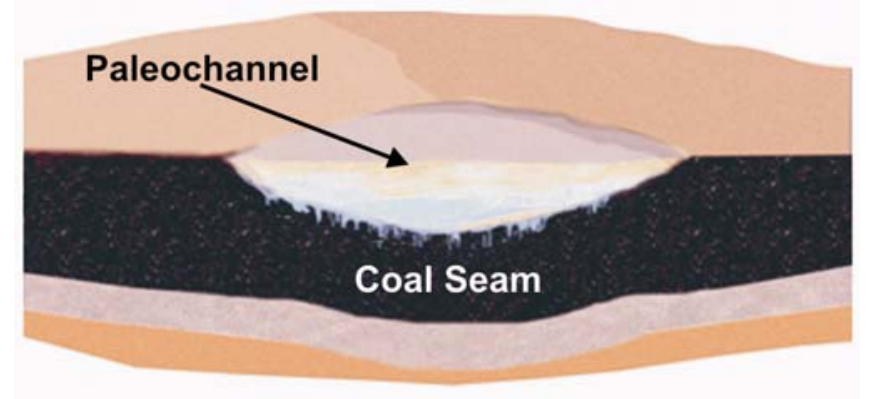

Figure 4-48. Naturally occurring undulations in coal seams caused by an overlying paleochannel.

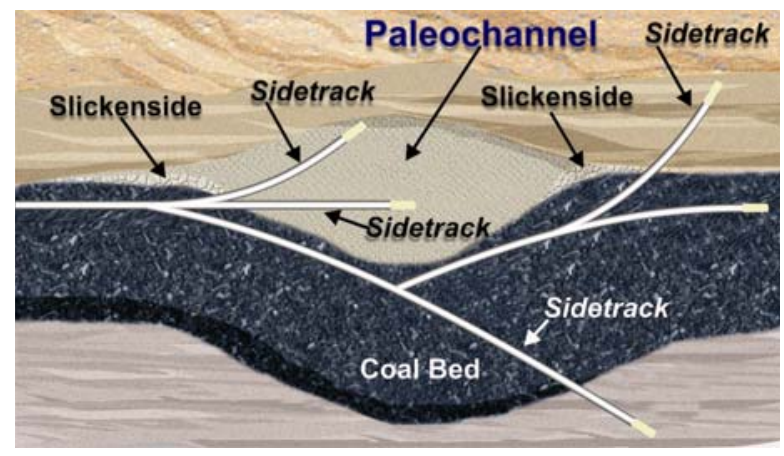

Figure 4-49. Sidetracks found in horizontal drilling in an undulating coal seam for coal bed methane extraction. 
Figure 4-50 depicts drilling into the gradational boundary of a coal seam using conventional drilling technology and horizontal drilling with the prototype DSR tool. Clay, heavy metal contaminants, and small coal particles are transported in the drilling mud cutting fluid, forming a filter cake that will reduce or completely shut down a CBM production well. Figure 4-51 shows an "eagle track" drilling pattern and methane extraction method that can be achieved using radarassisted geosteering, such as the prototype DSR tool.

Figure 4-50. Comparison of conventional horizontal drilling and radar-controlled drilling in a coal seam.

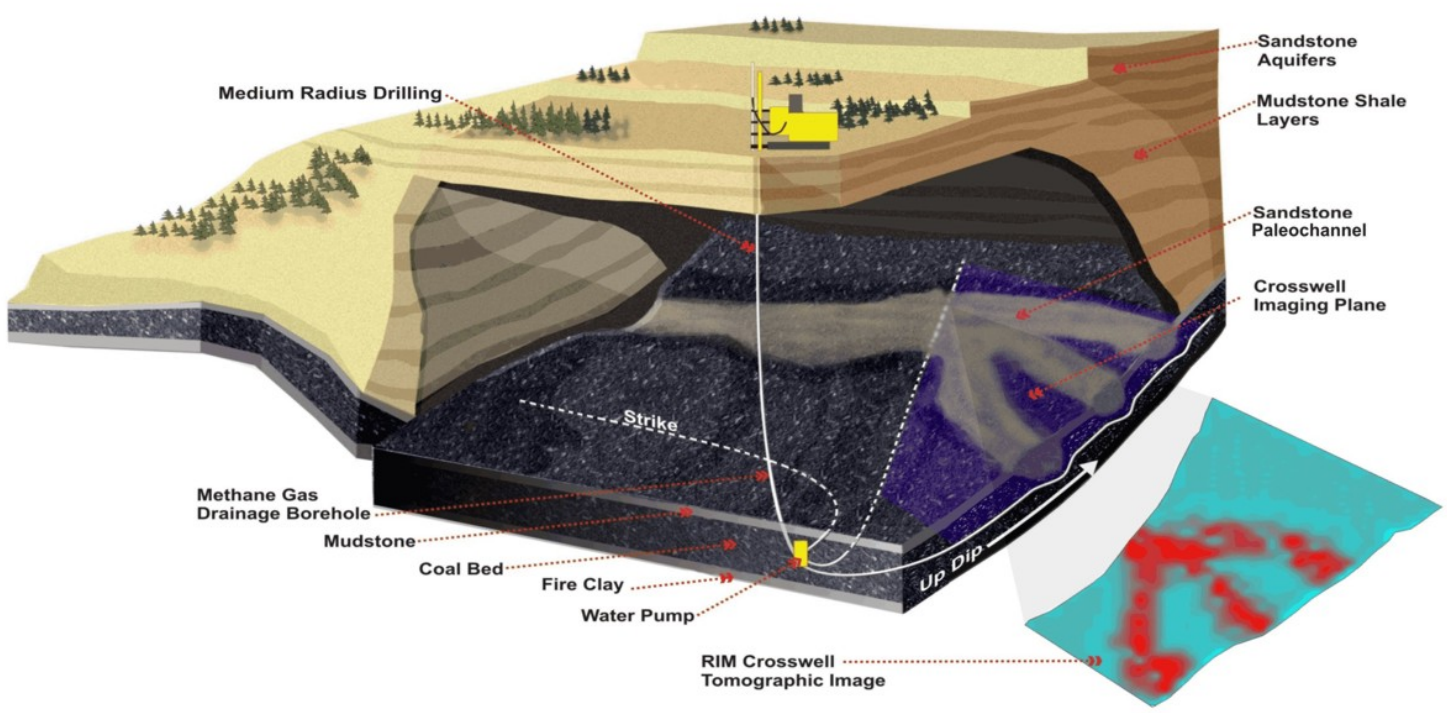

Figure 4-51. Radar-assisted eagle track drilling in coal seams.

\subsection{Conclusions}

A pre-commercial prototype drill string radar (DSR) tool has been designed, fabricated, and tested in both laboratory and field settings. The design of the DSR tool provides geosteering 
functionality for the oil and gas industry using coiled tubing or standard drill rod. Furthermore, the DSR tool can be integrated with the Stolar Data Transmission System (DTS) to provide realtime communications with the surface at high data rates to continuously orient the drilling process in real-time measurement-while-drilling (MWD) applications (see Figure 4-52). The DSR tool is designed to meet the requirements of a wide variety of MWD techniques and the bottom hole assembly can be easily configured to mate with conventional drilling hardware. Additional work to make the prototype DSR tool design more rugged to withstand the wide range of downhole conditions encountered in both coiled tubing and conventional drilling is ongoing.

Figure 4-52. Stolar Drill String Radar and Data Transmission System in a coiled tubing drilling application.

The Stolar DSR tool design has a number of important and distinguishing characteristics:

- propagates low- and medium frequency radio waves through sedimentary rock or hydrocarbon layers;

- operating frequency and dynamic range depend on the electrical properties of the host rock;

- measures dielectric constant and distance to boundary rock with a design range of approximately 40 feet;

- determines sedimentary rock characteristics and thickness of a hydrocarbon reservoir

- operates as a true radar device;

- suppression algorithms eliminate clutter from near-field or borehole wall reflections while enhancing reflections of more distant targets;

- low-frequency phase-modulated compound antennas suppress back lobes and generate directional EM fields; and

- electromagnetic fields are steerable electronically allowing the DSR tool to look up and down without rotating the drill rod. 
The Stolar DSR technology has a wide variety of applications, including:

- most geological and geotechnical drilling applications, including exploration drilling;

- conventional oil and gas reservoir production drilling, particularly horizontal drilling;

- horizontal CBM drilling, especially in thin coal seams; and

- drainage holes for CMM production, particularly in long wall mining environments. 


\subsection{References}

1. L.G. Stolarczyk, "Emergency and Operational Low and Medium Frequency Band Radio Communications System for Underground Mines," IEEE Transactions on Industry Applications 27, 780-790, August 1991

2. J.R. Wait and D.A. Hill, "The Theory of Electromagnetic Waves Along a Drill Rod in Conducting Rock," IEEE Transactions on Geoscience Electronics 17, 21-24, April 1979.

3. D.M. Pozar, Microwave Engineering, Addison-Wesley Publishing Company, Reading, Massachusetts, 1993.

4. M. Schwartz, Information Transmission, Modulation, and Noise, $3^{\text {rd }}$ Edition, McGraw-Hill Book Company, New York, 1980.

5. E.I. Parkhomenko, Electrical Properties of Rocks, Plenum Press, New York, 1967.

6. J.C. Cook, "Radar Transparencies on Mine and Tunnel Rocks," Geophysics 40, 865-885, 1975.

7. S. Feng and P.N. Sen, "Geometrical Model of Conductive and Dielectric Properties of Partially Saturated Rocks,” Journal of Applied Physics 묘, 3236-3243, 1985.

8. D. Middleton, Introduction to Statistical Communications Theory, Peninsula Publishing, Los Altos, California, 1987.

9. D. Gardner, J. Guerrier, and M. Martinez, "Ground Penetrating Radar Coal Measurements Demonstration at the U.S. Bureau of Mines Research Center, Pittsburgh, Pennsylvania," Final Report, EGG 11265-3015 UC-906, 1994.

10. M.L. Skolnik, Introduction to Radar Systems, McGraw-Hill Book Company, New York. 1962.

11. G. V. Keller and F. C. Frischknecht, Electrical Methods in Geophysical Prospecting, Pergamon Press, London, England, 1966. 